\title{
Quantum chemical studies of light harvesting
}

\author{
Carles Curutchet*a and Benedetta Mennucci*b \\ aDepartament de Fisicoquímica, Facultat de Farmàcia, Universitat de Barcelona, Av. \\ Joan XXIII s/n, 08028 Barcelona, Spain \\ bipartimento di Chimica e Chimica Industriale, University of Pisa, via G. Moruzzi \\ 13, 56124 Pisa, Italy
}

\section{Corresponding Authors:}

carles.curutchet@ub.edu, benedetta.mennucci@unipi.it

\begin{abstract}
The design of optimal light harvesting (supra)molecular systems and materials is one of the most challenging frontiers of science. Theoretical methods and computational models play a fundamental role in this difficult task, as they allow the establishment of structural blueprints inspired by natural photosynthetic organisms that can be applied to the design of novel artificial light-harvesting devices. Among theoretical strategies, the application of quantum chemical tools represents an important reality that has already reached an evident degree of maturity, although it still has to show its real potentials. This review presents an overview of the state of the art of this strategy, showing the actual fields of applicability but also indicating its current limitations, which need to be solved in future developments.
\end{abstract}

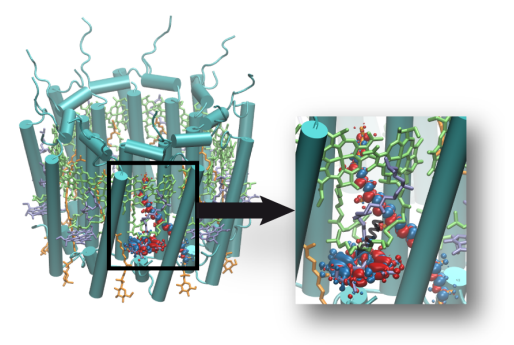

\section{Contents}

1. Introduction 3

2. Site energies

2.1 Quantum chemical methods 6

2.2 Environment effects 9

2.2.1 QM/classical models 10

2.2.2 Electrostatic shift method 15

2.2.3 QM description of the environment 17

3. Electronic couplings 20 
3.1 Singlet-singlet energy transfer (SET) 22

3.1.1 Methods based on transition densities $\quad 22$

3.1.2 Environment effects 26

3.1.2.1 QM/classical models $\quad 28$

3.1.2.2 QM/QM descriptions 30

3.1.3 Bridge effects, through-bond and charge transfer 31

3.1.4 Beyond the Coulomb coupling: the diabatization strategy 33

3.2 Triplet-triplet energy transfer (TET) 35

3.2.1 Fragment spin density (FSD) 36

$\begin{array}{ll}3.2 .2 \text { Environment effects } & 37\end{array}$

4. Spectral densities, disorder and correlations 38

4.1 MD-based approaches $\quad 42$

4.2 NMA-based approaches $\quad 45$

4.3 Correlations $\quad 46$

5. Applications 47

$\begin{array}{ll}\text { 5.1 Artificial light harvesting systems } & 47\end{array}$

5.1.1 Bridged dyads and dendrimers 48

5.1.2 Conjugated polymers and organic crystals 53

5.1.3 Quantum dots and plasmonic nanoparticles $\quad 57$

$\begin{array}{ll}5.2 \text { Biological light harvesting systems } & 61\end{array}$

5.2.1 LH1 and LH2 62

5.2.2 Chlorosome and FMO 66

$\begin{array}{ll}\text { 5.2.3 Phycobilisome and phycobiliproteins } & 71\end{array}$

5.2.4 PCP and FCP 74

$\begin{array}{ll}5.2 .5 \text { LHC family } & 76\end{array}$

5.2.6 Reaction centers $\quad 79$

6. Conclusions and outlook 83 


\section{INTRODUCTION}

Nature has developed sophisticated and highly efficient molecular architectures to absorb sunlight and convert it into chemical energy, which is finally used by photosynthetic organisms (bacteria, algae or plants) to live and grow. The comprehension of these light-harvesting (LH) processes occurring in photosynthetic pigment-protein complexes (PPC) in terms of the pigment disposition in the protein scaffold has been an important goal since the first high-resolution structure of the Fenna-Matthews-Olson (FMO) complex appeared 40 years ago. ${ }^{1}$ Beyond the prime importance of their role in photosynthesis, PPCs also represent ideal model systems to assess theoretical models of light harvesting and therefore improve our understanding of the physical principles underlying these phenomena in multichromophoric systems. In this sense, a key advantage is the known and relatively fixed arrangement of the pigments in many protein complexes. Indeed, a considerable variety of crystal structures have been solved in the last decades, including highly-symmetric systems like the lightharvesting complex 2 (LH2) of purple bacteria or less ordered ones like the major lightharvesting complex II (LHCII) of plants. Such structural variability, much larger for antenna PPCs than it is for reaction centers (RC), has flourished as a consequence of the evolutionary pressure aimed at matching their absorption spectra with the spectra of available sunlight in different habitats, particularly reduced in marine and freshwater environments. Another advantage of PPCs as models of light-harvesting is the fact that the initial steps of photosynthesis, the capture of sunlight by antenna complexes and the transfer of the absorbed energy to RCs, where charge separation is realized, are typically achieved with extremely high efficiencies.

A great amount is known now about natural PPCs and the physics at the basis of their electronic energy transfer $(E E T)^{2-21}$ processes so that, in principle, it should be possible to construct effective artificial analogues. Such materials could be used as organic solar concentrators, sensitisers for solar cells, unnatural neural networks, opto-electronic components or advanced signaling devices. Unfortunately, the practice of such artificial systems is still quite far from the expected efficiencies and stabilities required for largescale manufacturing.

It is therefore of paramount importance to combine our knowledge of the physical processes at the bases of the LH function in natural systems with an optimal design and an efficient realization of artificial structures, and finally insert them within a proper device. This three-step strategy obviously needs to combine know-hows from very different fields going from structural biology to nano-engineering. Evidently chemistry plays a pivotal role in this strategy as the most natural way to look at molecular transformations. In particular, we can expect real advances if accurate molecular-based theories will be transformed into efficient computational tools capable of simulating in a coherent and comparable way natural PPCs and artificial systems. Along this research line, quantum chemistry is clearly the "ideal" method due to its versatility and 
extensibility both towards different length scales when combined to classical models, and time scales when generalized to $a b$ initio and classical molecular dynamics simulations.

Ideally, one would like to model the light-harvesting process by performing quantumchemical calculations of the photoinduced process in the full system, and then simulating the quantum dynamics of the resulting excited-states, for example, from nonadiabatic molecular dynamics simulations. ${ }^{22-26}$ This approach not only would give access to the energy transport dynamics but it would also allow an accurate simulation of the optical spectra of the system, which dictates its spectral cross-section for light absorption, a key aspect of any LH material, and the backbone of our experimental knowledge of natural photosynthetic complexes. Unfortunately, the complexity of LH systems (especially those present in photosynthetic organisms) and the considerable size of the network of interacting molecular units (the chromophores) have so-far precluded the direct application of this approach. Nevertheless, accurate descriptions can still be obtained, e.g. by introducing an exciton picture combined with quantum master equation approaches.

Within this framework, the electronic Hamiltonian that describes the eigenstates of the multichromophoric system is approximated in terms of the excitations of the single chromophores (the site energies $\varepsilon$ or diagonal elements) and the corresponding electronic couplings among them ( $V$ or off-diagonal elements), namely:

$$
H_{S}=\sum_{i}^{N} \varepsilon_{i}|i\rangle\left\langle i\left|+\sum_{i \neq j}^{N} V_{i j}\right| i\right\rangle\langle j|
$$

where, for the sake of clarity, we have assumed $N$ interacting chromophores each one contributing with only one excitation.

The excitonic system is then placed within a "bath" (or reservoir) which, in a chemical language, can be described as the set of vibrations (of intra/ inter-chromophore as well as chromophore-environment nature) that couple to the excitations. Such a coupling is generally described in terms of the system-bath spectral densities and the associated reorganization energies.

Once there is a reliable estimate of the excitonic parameters (site energies and electronic couplings) and spectral densities, the total Hamiltonian governing the dynamics of the system and the bath can be written as:

$$
H=H_{S}+H_{B}+H_{S B}
$$

where $H_{B}$ represents the Hamiltonian of the bath (usually approximated as a set of harmonic oscillators) whereas $H_{S B}$ describes the system-bath interaction.

It is exactly the interaction between the system and the bath which governs the energy transport and defines the EET regime. ${ }^{9,20,27,28}$ Depending on the relative strengths of the electronic coupling and the coupling to the bath, we go from a Förster-type EET with a 
rate that is proportional to the square of the electronic coupling, assumed to be much weaker than the reorganization energy, to the opposite Redfield regime where instead the excitonic couplings are much larger than the coupling with the bath and the process is dominated by relaxation instead of EET. It is in between these two limiting cases that most natural and artificial systems seem to operate and reach their maximum efficiency. ${ }^{29,30}$ In this intermediate regime, the timescale characterizing the bath relaxation is on the order of the transfer process, and the Born-Oppenheimer approximation is no longer valid. The electronic and nuclear degrees of freedom cannot be factorized and bath correlations come into play. In that case, nonperturbative numerical methods for accurately computing quantum dissipative dynamics ${ }^{31,32}$ become the methods of choice.

From this brief resume, it is evident that for an accurate description of light-harvesting processes in both artificial and natural systems three are the necessary ingredients: the site energies, the couplings and the spectral densities. While in the past simplified models combined with experimental data were largely used to get an estimate of them, in the last ten years quantum chemical methods have shown to be an extremely powerful approach. They in fact allow simulating systems going from simple donoracceptor dyads to complex multichromophoric complexes. Moreover, when combined with proper embedding models, an accurate description of the important effects exerted by the surrounding environment can also be achieved.

Here, we review the current state of the art of quantum chemical approaches for the modeling of light-harvesting processes and their applications to artificial and natural systems. We start from the description of the theoretical methods and computational models developed so far for the calculation of (i) site energies, (ii) electronic couplings and (iii) spectral densities. We continue with the presentation and discussion of selected examples of applications of the same methods and we end with some conclusions and possible future directions.

\section{SITE ENERGIES}

The starting point for any quantum chemical simulation of EET in supramolecular systems is the evaluation of the excitation energies of the involved absorbing units. Such quantities, generally indicated as "site energies", are in fact the central parameters (together with the electronic couplings) of the excitonic analysis. The calculation of site energies should in principle be possible by applying any QM method available in the literature for treating electronically excited states of molecular systems. However, there are three specificities of EET supramolecular systems which generally reduce the choice to few QM approaches. The first specificity is that the chromophoric "units" (pigments) are generally large (normally they contain more than 90 atoms): this prevents the use of very accurate but costly QM methods for production purposes whereas examples are available in the literature for benchmarking studies. The second specificity is that site 
energies can largely be affected by the surrounding environment: the QM method of choice has thus to be coupled with some model which can properly take into account environment effects either at QM or classical level. Finally, possible couplings with the vibrations of the pigment as well as of its environment can play a fundamental role: the modeling has thus to account for electron-phonon interactions. Here below the first two specificities will be presented and commented while to the third one we shall devote section 4 .

\subsection{Quantum chemical methods}

At the beginning of the quantum chemical modeling of EET in natural LH systems, the only feasible methods were of semiempirical nature. For long time the method of choice was the configuration interaction (CI) approach based on the Pariser-Parr-Pople (PPP) model Hamiltonian, ${ }^{33,34}$ while later CIS (configuration interaction with single excitations) approaches coupled to the Zerner's intermediate neglect of differential overlap (ZINDO) ${ }^{35}$ Hamiltonian became the common choice. CIS has also been largely used in combination with the ab initio Hartree-Fock (HF) Hamiltonian-However, CIS energies are simply not accurate since they employ molecular orbitals optimized for the ground state. The correlation energy introduced by the CI among all singly excited states is too small to remedy this orbital deficiency, so that the excited states are too high in energy compared to the ground state. Recently, variations of the CIS method have been proposed with the goal of keeping its easiness of use but improving the accuracy. An example is the perturbatively orbital optimized CIS (OO-CIS) method, which has been recently developed to get a significant correction to charge-transfer (CT) excited state energies for which standard CIS typically leads to an overestimation of $1-2 \mathrm{eV} .{ }^{36}$ This and other variations of CIS have shown to be quite successful in the modeling of electron and energy transfer. ${ }^{37}$

With the diffusion of Density Functional Theory (DFT) for quantum chemistry and its extension to excitation processes within the linear response Time-Dependent DFT (TDDFT), the situation has rapidly changed and both semiempirical and CIS methods have been definitely superseded even if they still continue to be used especially in the field of exciton transport in materials (see section 5.1).

TDDFT, contrary to CI-like approaches, does not explicitly calculate the two states of interest (e.g. the initial and the final one in the investigated transition) but it obtains the transition energies directly through a linear-response approach (i.e., as poles of the dynamic polarizability of the system). By adopting the formalism derived by Casida, ${ }^{38}$ the excitation energies can be obtained as eigenvalues of a non-Hermitian problem of the type: 


$$
\begin{aligned}
& {\left[\begin{array}{cc}
\mathbf{A} & \mathbf{B} \\
\mathbf{B}^{*} & \mathbf{A}^{*}
\end{array}\right]\left[\begin{array}{c}
\mathbf{X} \\
\mathbf{Y}
\end{array}\right]=\omega\left[\begin{array}{cc}
\mathbf{1} & \mathbf{0} \\
\mathbf{0} & -\mathbf{1}
\end{array}\right]\left[\begin{array}{l}
\mathbf{X} \\
\mathbf{Y}
\end{array}\right]} \\
& A_{i a, j b}=\delta_{i j} \delta_{a b}\left(\varepsilon_{a}-\varepsilon_{i}\right)+(i a \mid j b)+\left(i a\left|f_{x c}\right| j b\right) \\
& B_{i a, j b}=(i a \mid b j)+\left(i a\left|f_{x c}\right| b j\right)
\end{aligned}
$$

The leading term on the diagonal of the A matrix is the difference of the energies of the Kohn-Sham orbitals $i$ and $a$, which are the ones from which and to which the electron is excited, respectively. The second term of the A matrix and the elements of the $\mathbf{B}$ matrix stem from the linear response of the Coulomb and exchange/correlation $(x c)$ operators to first-order changes in the orbitals. Usually the adiabatic local density approximation (ALDA) is introduced: the originally non-local (in time) time-dependent $x c$ kernel is replaced with a time-independent local one based on the assumption that the density varies only slowly with time. This approximation allows the use of a standard local ground-state $x c$ potential in the TDDFT: the response of the $x c$ potential corresponds to the second functional derivative of the exchange-correlation energy, which is also called the $x c$ kernel, and is given as

$$
\left(i a\left|f_{x c}\right| j b\right)=\int d \mathbf{r} d \mathbf{r}^{\prime} \phi_{i}^{*}(\mathbf{r}) \phi_{a}(\mathbf{r}) \frac{\delta^{2} E_{x c}}{\delta \rho(\mathbf{r}) \delta \rho\left(\mathbf{r}^{\prime}\right)} \phi_{b}^{*}\left(\mathbf{r}^{\prime}\right) \phi_{j}\left(\mathbf{r}^{\prime}\right)
$$

A common approximation to the TDDFT eigenvalue problem is the Tamm-Dancoff approximation (TDA), which is the TDDFT analog of CIS: it corresponds to neglecting the matrix B. This leads to a Hermitian eigenvalue equation

$$
\mathbf{A X}=\omega \mathbf{X}
$$

where the definition of the matrix elements of $\mathbf{A}$ is still the same as TDDFT. It is worthwhile to note that TDA/TDDFT is usually a very good approximation to TDDFT. Moreover, TDA avoids certain kinds of triplet near-instabilities which are instead present in TDDFT. ${ }^{39}$

In the years, TDDFT has shown to be a very effective approach to describe electronic excitations in medium-to-large molecules and it has become extremely popular within the modeling of both natural and artificial LH systems. However, along with its increasing applications, TDDFT has also shown its intrinsic limitations.

A well-known pitfall of most TD-DFT functionals which becomes relevant in the modeling of LH systems is the overestabilization of charge-transfer (CT) like excitations. This problem is particularly important in supermolecular calculations including several pigments, given that Frenkel excitons can coexist with CT like excitations involving the transfer of charge from one pigment to another. In these cases, 
it is now well established that the long-range-corrected (LC) and Coulomb-attenuating methods (CAM) have to be preferred with respect to other types of functionals. ${ }^{40,41}$ In these schemes, the two-electron repulsion operator $1 / \mathrm{r}_{12}$ in the exchange functional is divided into short- and long-range parts using the error function,

$$
\frac{1}{r_{12}}=\frac{1-\left[\alpha+\beta \operatorname{erf}\left(\omega r_{12}\right)\right]}{r_{12}}+\frac{\alpha+\beta \operatorname{erf}\left(\omega r_{12}\right)}{r_{12}}
$$

with three parameters, $\alpha, \beta$, and $\omega$ which in the $\mathrm{LC}$ reduce to one as $\alpha=0$ and $\beta=1$. The DFT exchange functional is included through the first term, and the long-range orbital-orbital exchange interaction is described using the Hartree-Fock exchange integral. The distance-dependent ratio of these two terms is determined by the rangeseparated parameter $\omega$. Various LC and CAM functionals have been developed, among them we cite here the LC- $\omega$ PBE, ${ }^{42}$ the CAM-B3LYP,${ }^{43}$ and $\omega B 97 X .{ }^{40}$ The parameters $\alpha, \beta$, and $\omega$, have been determined to minimize the mean error of a benchmark database, which generally consists of experimental data or high-level calculation results of atoms and small molecules. Recent studies show, however, that such parameters are not always appropriate for describing excited states of large molecules as those involved in artificial and natural LH systems but they need to be optimally tuned. ${ }^{44-46}$ Recently, the development of both the constricted variational DFT (CV-DFT) ${ }^{47}$ and the constrained DFT (CDFT) method, ${ }^{48}$ have provided alternative ways to compute charge-transfer states within DFT. In particular, in CDFT a Lagrange multiplier term is added to the Kohn Sham energy functional, thereby enforcing an additional constraint on the electronic density. This theoretical methodology has also been applied to the evaluation of charge on-site energies and transfer integrals in supramolecular architectures based on representative materials for the organic light-emitting diode (OLED) technology. ${ }^{49} \mathrm{~A}$ variant of the constrained DFT to describe the energetics of long-range chargeseparation processes has been also proposed within the subsystem DFT formulation (see section 2.2.3). ${ }^{50}$

During the years, TDDFT has shown the best cost/accuracy ratio, for example, when applied to the typical porphyrine-like pigments common in natural and artificial LH systems. ${ }^{51}$ However, TDDFT, as any other single reference method, cannot properly describe double excited states. This is a relevant limit especially in another important class of LH pigments, that of carotenoids (Car). The nature of the Car electronically excited states can be represented using an ideal picture of a conjugated $\pi$-system in a perfect $\mathrm{C}_{2} \mathrm{~h}$ point-group symmetry. According to this picture, the lowest lying singlet state $\left(\mathrm{S}_{1}\right)$ is described by the same irreducible representation as the ground state $\left(\mathrm{Ag}_{\mathrm{g}}{ }^{-}\right)$, and the corresponding one-photon transition is forbidden, while the second singlet excited state $\left(\mathrm{S}_{2}\right)$ has $\mathrm{B}_{\mathrm{u}}{ }^{+}$symmetry and it is the one determining the strongly allowed absorption. An additional $\left(\mathrm{S}_{3}\right)$ dark state of $\mathrm{B}_{\mathrm{u}}{ }^{-}$symmetry is also expected. It is known that the these excited states have a rather different multireference character, $\mathrm{S}_{2}$ being largely dominated by a single HOMO-LUMO excitation while $\mathrm{S}_{1}$ (and $\mathrm{S}_{3}$ ) present a 
strong double excitation character. As a result, single-reference approaches like TDDFT only properly describe $\mathrm{S}_{2}$ (which becomes the lowest singlet state) while they cannot give the correct energy position and character of the $S_{1}$ and $S_{3}$ states. For those states, a multireference approach is necessary and the multireference configuration interaction extension of density functional theory (DFT/MRCI $)^{52}$ has shown to be a quite effective formulation. Recently, ${ }^{53}$ it has been shown, however, that the combination of the TDA approximation with meta-GGA DFT functionals (and in particular TPSS) ${ }^{54}$ can give a generally good description of the transition energies of all the three lowest singlet states of Cars of different length. This unexpected good behavior was shown to be due to a cancellation of the effects that the neglect of the multideterminant character has on the ground and the excited states (this also explains why TDA gives a better picture than time-dependent DFT). From the same study, it also appeared that this cancellation does apply not only to transition energies but also to transition densities (and transition dipoles), for which a general good reproduction of DFT/MRCI results was obtained. These findings suggest that the fast and size-extensible TDA can represent an effective approach to study EET involving Cars.

In the last few years, an alternative formulation of TDDFT has gained prominence for simulating optical responses of molecular chromophores. ${ }^{55,56}$ Rather than solving for the eigenvalues of eq.(1.2), this real-time (RT) TDDFT ${ }^{57-60}$ simulates the absorption spectrum by propagating the density in time and computing frequencies and intensities from the Fourier transform of time-dependent dipole moment. In addition, RT-TDDFT has been used to simulate time-dependent processes such as exciton diffusion ${ }^{61}$ and charge-separation in light-harvesting systems. ${ }^{62}$

Along the years, accurate QM methods going beyond TDDFT have also been applied to LH systems, mostly as benchmarking tests. However, several studies recently appeared in the literature show that highly correlated QM methods such as Coupled Cluster $(\mathrm{CC})^{63,64}$ or second-order perturbation theory ${ }^{65}$ are no longer prohibitive for detailed analyses of LH systems of real applicative or biological interest. However, their computational cost remains quite demanding and we can expect that the golden standard for the next years will remain the TDDFT approach when combined to a well chosen functional.

\subsection{Environment effects}

A well-known strategy to apply QM descriptions to complex systems made of different interacting components is to introduce a hybrid energy expression based on the separation between an "active region" $(A)$ and the "environment" $(E)$, namely:

$$
E^{\text {Hybrid }}=E_{A}(M A)+E_{E}(M E)+V_{E A}
$$


where $M A$ and $M E$ indicates the methods used to describe the subsystem $A$ and $E$, respectively and $V_{E A}$ is the interaction energy. This approach is valid when the property or process of interest can be localized in the subsystem $A$ without loosing or modifying any aspect of it. At the same time, however, the complementary subsystem $E$ is not a passive spectator but it generally affects the properties of $A$ through different physical effects which are collected in the term $V_{E A}$.

This strategy is not limited to the ground state of $A$ but it can be extended to electronic excited states and used to get transition energies and properties. One can in fact generalize eq 5.2 to the two electronic states involved in the excitation (which has to be localized on $A$ ). Since the electron density of system $A$ changes upon excitation, the interaction between systems $A$ and $E$ will be different in the two electronic states; moreover the same density will be affected by the presence of the interaction with $E$; as a result of these two effects the transition energy (and the related transition properties) will be different with respect to the case of the system $A$ alone.

Several combinations of different methods are possible for $A$ and $E$; the most common ones are described here below.

\subsubsection{QM/classical models}

A very successful strategy is to combine the QM description of the $A$ subsystem with a classical one for the $E$ subsystem. This strategy can be formulated either using a molecular mechanics (MM) force field or a continuum dielectric (CD) to model the classical system.

In both cases we can introduce a similar QM picture in terms of an effective Hamiltonian giving rise to an effective Schrödinger equation for $A$. Introducing the standard Born-Oppenheimer approximation, the electronic wavefunction of $A$ will satisfy the following equation:

$$
\hat{H}^{e f f}|\Psi\rangle=\left(\hat{H}^{0}+\hat{H}^{Q M / c l a s}\right)|\Psi\rangle=E|\Psi\rangle
$$

where $H^{0}$ is the Hamiltonian of the isolated $A$ system and the operator $\mathrm{H}^{\mathrm{QM} / \mathrm{class}}$ introduces the coupling between $A$ and $E$.

The form of the operator $H^{\mathrm{QM} / \mathrm{class}}$ depends on the particular method used. In the most common formulations, the coupling term between the QM and the classical subsystems is limited to the electrostatic interaction generally described in terms of point-like charges:

$$
\hat{H}^{Q M / c l a s} \rightarrow \hat{H}^{e l e}=\sum_{m} q_{m}\left(\mathbf{r}_{m}\right) \hat{V}\left(\mathbf{r}_{m}\right)
$$


where $V\left(\mathbf{r}_{m}\right)$ is the electrostatic potential operator due to the electrons and nuclei of $\mathrm{A}$ acting on the "environment" represented in terms of point charges $\left(q_{m}\right)$.

All nonelectrostatic interactions are generally introduced as corrections to the eigenvalues of the effective Hamiltonian and they do not affect the wavefunction of $A$.

Even if the operator reported in eq 7.2 is equivalent in $\mathrm{QM} / \mathrm{MM}$ and $\mathrm{QM} / \mathrm{CD}$ formulations, the meaning of the "environment" charges is completely different.

In standard "electrostatic embedding" formulations of QM/MM they are fixed atomic parameters extracted from the selected MM force field. On the contrary, in $Q M / C D$ approaches, they are no more linked to the positions of the environment atoms but they are placed on the surface of a cavity which is assumed to contain the subsystem $A$. Within this framework, such charges are not fixed parameters but they are induced by the electrostatic potential due to $A$ : in other words they represent the polarization of the environment. This formulation is common to all continuum models which adopt an apparent surface charges (ASC) formulation such as the various versions of the polarizable continuum model (PCM) developed in $\mathrm{Pisa}^{66}$ (the original or D-PCM, the IEF-PCM ${ }^{67}$ and the C-PCM ${ }^{68}$ ), the SS(V)PE (Surface and Simulation of Volume Polarization for Electrostatics) model developed by Chipman $^{69}$ and the Conductor-like Screening Model (COSMO) developed by Klamt. ${ }^{70}$

Due to the different definition of the environment charges in the two QM/classical models, the corresponding $H^{e l}$ operator has different implications in the way we solve eq 6.2. In $\mathrm{QM} / \mathrm{MM}$ it simply adds a further one-electron term to the Hamiltonian $H^{0}$ exactly as the electron-nuclei attraction. In QM/CD models, instead, $H^{e l}$ becomes non linear with respect to the wavefunction, as the charges $q$ depend on the electronic density $\left(\rho_{A}\right)$ of $A$; they are generally obtained as the solution of a matrix equation of the type:

$$
\mathbf{T}(\varepsilon, \Gamma) \mathbf{q}=\mathbf{R}(\Gamma) \mathbf{f}\left(\rho_{M}\right)
$$

where $\mathbf{q}$ is the vector collecting the ASC-charges, $\mathbf{T}$ and $\mathbf{R}$ are square matrices depending on the topology of the cavity surface $(\Gamma)$, the mesh used to sample it and the dielectric constant $(\varepsilon)$. The vector $\mathbf{f}$ instead collects the source of the dielectric polarization, i.e. the field (or the potential according to the different formulations) generated by $A$ on the cavity surface. As a result of eq 8.2 the solution of eq 6.2 requires an iterative scheme in which the polarization of $A$ is coupled to that of $E$. This mutual polarization effects are easily solved if the QM method already implies an iterative solution such as in self-consistent field (SCF) formulations of HF or KS-DFT.

Also QM/MM descriptions can be extended to include mutual polarization between $A$ and $E$; this extension is generally called "polarizable embedding" and it can be achieved using induced dipoles or fluctuating charges, ${ }^{71}$ or introducing a Drude-like formulation. ${ }^{72}$ 
Due to its many applications in the field of natural and artificial LH systems, we focus here on the polarizable embedding which uses induced dipoles: within this framework, each MM site is described as an atomic point charge and a polarizability $\boldsymbol{\alpha}$. As a result, the QM/class Hamiltonian reported in eq 6.2 becomes:

$$
\hat{H}^{Q M / c l a s s}=\hat{H}^{e l e}+\hat{H}^{p o l}=\sum_{m} q_{m}\left(\mathbf{r}_{m}\right) \hat{V}\left(\mathbf{r}_{m}\right)-\sum_{m} \boldsymbol{\mu}_{m}\left(\mathbf{r}_{m}\right) \cdot \hat{E}\left(\mathbf{r}_{m}\right)
$$

In eq $9.2 V\left(\mathbf{r}_{m}\right)$ and $\boldsymbol{E}\left(\mathbf{r}_{m}\right)$ represent the electrostatic potential and the electric field operators due to the QM $A$ system calculated at the MM sites.

The dipoles induced on each MM polarizable site can be obtained assuming a linear approximation, neglecting any contribution of magnetic character, and using an isotropic polarizability for each selected point in the MM part of the system. The electric field that determines such dipoles contains a sum of contributions from the QM subsystem, from the MM point charges and from the induced dipole moments. This mutual polarization between the dipoles can be solved by introducing a matrix equation of the type:

$$
\mathbf{R}(\alpha) \mu=\mathbf{E}^{Q M}+\mathbf{E}^{q^{M M}}
$$

where the vectors $\mathrm{E}^{\mathrm{QM}}$ and $\mathrm{E}^{q M M}$ collect the electric field from the $\mathrm{QM}$ subsystem and the $\mathrm{MM}$ permanent charge distribution, respectively. The form of matrix $\mathbf{R}$ is determined uniquely by the position of the polarizable MM sites and the values used for the point polarizabilities $\alpha_{i}$, namely:

$$
\mathbf{R}=\left[\begin{array}{cccc}
\alpha_{1}^{-1} & 0 & \cdots & 0 \\
0 & \alpha_{2}^{-1} & \cdots & 0 \\
\vdots & \vdots & \ddots & \vdots \\
0 & 0 & \cdots & \alpha_{N}^{-1}
\end{array}\right]+\left[\begin{array}{cccc}
0 & T_{12} & \cdots & T_{1 N} \\
T_{21} & 0 & \cdots & T_{2 N} \\
\vdots & \vdots & \ddots & \vdots \\
T_{N 1} & T_{N 2} & \cdots & 0
\end{array}\right]
$$

where $N$ is the number of polarizable sites. The dipole field tensor $\mathrm{T}_{\mathrm{pq}}$ is defined as

$$
T_{p q}=\frac{\mathbf{1}}{r_{p q}^{3}}-\frac{3}{r_{p q}^{5}}\left[\begin{array}{ccc}
x^{2} & x y & x z \\
y x & y^{2} & y z \\
z x & z y & z^{2}
\end{array}\right]
$$

where $\mathrm{r}_{\mathrm{pq}}$ is the distance between site $p$ and $q$, and $x, y$ and $z$ are the Cartesian components of the vector connecting the two.

It is evident that the results of the method critically depend on the quality of the parameterization and in particular on the point polarizabilities usually associated with 
atoms. The atomic polarizability parameters are generally obtained by fitting to either experimental or QM molecular polarizabilities or QM electrostatic potentials. The parameterization methods can be formulated in two ways: "additive" and "interactive" models, depending on the level of interactions permitted between induced dipoles. ${ }^{73}$ In the "additive" approach, polarizable sites are allowed to respond to an external electric field but not to permanent and induced multipoles on other sites within a molecule. In nonadditive, also called "interactive", polarization models, instead, each polarizable site is allowed to respond to an external electric field not only from other molecules but also from other sites within the same molecule. Consequently, all interacting sites polarize themselves. Under certain conditions, two inducible dipoles at short distances can cause a "polarization catastrophe"; this effect can be imputed to the use of point polarizabilities instead of diffuse charge distributions. To avoid this problem, the 1-2 and 1-3 bonded polarization interactions are turned off; alternatively, one can apply distance-dependent damping functions for interactions on short distances or combine both procedures. Several schemes for damping interactions between inducible dipoles at short distances have been proposed by Thole by introducing distance-dependent screening functions to be used in the calculation of the Thole tensor of eq $12.2 .{ }^{74,75} \mathrm{We}$ also recall that the introduction of induced dipoles requires to re-parameterize the permanent charges: commonly this is done using an approach based on the fitting of the electrostatic potentials (ESP) calculated at QM level on a grid of points but now the fitting is done on the sum of the potential generated by induced dipoles and point charges. $^{76}$

In both polarizable $\mathrm{QM} / \mathrm{MM}$ and $\mathrm{QM} / \mathrm{CD}$, once solved eq $6.2, A$ (in its ground state) and $E$ will be mutually equilibrated. If now, we induce an excitation in $A$ this equilibrium is perturbed as the charge distribution of $A$ is rapidly changed. The differences in the characteristic response times of the electronic and nuclear degrees of freedom of the environment generally leads to a so-called nonequilibrium regime in which the inertial components (i.e., those arising from nuclear motions) are no longer equilibrated with $A$. The "nonequilibrium" regime will possibly relax into a new equilibrium in which all the degrees of freedom of the environment including the slow ones are fully relaxed. The energy difference between "equilibrium" and "nonequilibrium" regimes is generally known as solvent "reorganisation energy".

The "nonequilibrium" regime can be properly described within the continuum framework introducing a separation of the ASC charges into a dynamic contribution, mimicking the electronic component of the polarization, and an inertial contribution, due to the "nuclear" polarization. The dynamic charges are obtained by using the same expression (eq 8.2) used for the full equilibrium but this time the matrix $\mathbf{T}(\varepsilon, \Gamma)$ is calculated with the optical component $\left(\varepsilon_{\infty}\right)$ of the dielectric permittivity, and the QM electric field or electrostatic potential collected in the vector f refers to the new QM charge distribution (namely to the excited state in a vertical excitation process). By 
contrast, the inertial component is obtained as the difference of the full polarization and the dynamic component both calculated in equilibrium with the initial QM charge distribution.

Also $\mathrm{QM} / \mathrm{MM}$ methods in the electrostatic embedding formulation account for the inertial part of the nonequilibrium response: during the excitation process, in fact, the MM charges are kept frozen both in magnitude and position. To properly include the dynamic part of polarization, instead, a polarizable embedding is necessary to account for the electronic rearrangement of the environment upon excitation.

While the physical processes describing the environment response following a vertical excitation are physically well established their QM modeling is not univocal nor straightforward. During the years, different formulations have been proposed and tested. For the sake of clarity, it is useful to collect them in two main families, the so-called "linear response" (LR) and "state-specific" (SS) approaches. By simplifying at most, the excitation energy between an initial $(i)$ and final $(f)$ state of $A$ obtained within the two formulations can be written as:

$$
\omega_{i \rightarrow f}^{L R}=\omega_{i \rightarrow f}^{0}+R_{e q / n e q}\left(\rho_{i \rightarrow f}^{T}\right)
$$

or

$$
\omega_{i \rightarrow f}^{S S}=\omega_{i \rightarrow f}^{0}+R_{e q / n e q}\left(\Delta \rho_{i \rightarrow f}\right)
$$

where $\omega_{0}$ is equivalent in the two formulations and it represents a "fully frozen environment" contribution (no dynamic and no inertial relaxations are allowed) whereas $R$ is the correction due to the response of the environment which can be calculated either assuming an equilibrium or a nonequilibrium model.

In non polarizable QM/classical approaches (such as an electrostatic embedding $\mathrm{QM} / \mathrm{MM}$ ) only $\omega_{0}$ can be included as $R$ is null. In the LR formulation of polarizable embeddings (either continuum or atomistic), the response $R$ is computed from the transition density $\rho_{i \rightarrow f}^{T}$ characterizing the excitation in the $A$ subsystem, while in the SS approach the same response is determined by the difference in the electron density between initial and final states of $A, \Delta \rho_{i \rightarrow f}$.

The LR formulation (13.2) is the common one used in combination with TDDFT as the transition density from ground to excited state is exactly the quantity available from such a method. On the contrary, the SS scheme, requiring the change in the electron density due to the excitation, is naturally coupled with state-specific wavefunction formulations. As a matter of fact, $\Delta \rho_{\mathrm{gs} \rightarrow \mathrm{exc}}$ can be obtained also from TDDFT if its extension to analytical gradients (or Z-vector formulation) is used. TDDFT-SS formulations of PCM have been presented by Caricato et al. (corrected linear response, 
cLR), ${ }^{77}$ by Improta et al. ${ }^{78}$ and more recently by Marenich et al. (vertical excitation method, VEM). ${ }^{79}$ These SS-PCM formulations differ in the way they compute the last term in the right hand side of eq 14.2; this term in fact introduces a further iterative scheme on the excited state density (exactly as already described for the ground state). In both cLR and VEM this iterative scheme is applied to the change of the electron density $\left(\Delta \rho_{\mathrm{gs} \rightarrow \mathrm{exc}}\right)$. The difference between the two methods is that cLR approximates $\Delta \rho_{\mathrm{gs} \rightarrow \mathrm{exc}}$ to the first iteration while VEM obtains the full convergence between $A$ and $E$ responses. The method proposed by Improta et al. introduces a different iteration scheme directly on the full excited state density $\rho_{\mathrm{exc}}=\rho_{\mathrm{SCF}}+\Delta \rho_{\mathrm{gs} \rightarrow \mathrm{exc}}$ : the ground state contribution $\rho_{\mathrm{SCF}}$ is recalculated by iterating the KS-DFT equation in the presence of the PCM charges calculated on the excited state.

It has been shown that LR gives correct solvatochromic shifts for excitations characterized by a large transition dipole but a small change in the state density. On the contrary, for charge-transfer like excitations the LR scheme is inadequate because it neglects the electron density relaxation. ${ }^{80}$ These shortcomings however are generally limited in terms of absolute values (and in many cases almost negligible) as the $R$ term of eqs (13.2-14.2) is only determined by the dynamic part of the polarization. In the absorption process, in fact, the dominant term is $\omega_{0}$, i.e. the excitation energy associated to a completely frozen environment (represented by the ground-state polarization) while the response $R$ represents only a small correction.

In the discussion presented so far, the effect of the environment on the excitations has been limited to electrostatics plus polarization. Different attempts to go beyond this approximation have been proposed. Semiclassical expressions have been derived by Renger et al., under a dipole approximation ${ }^{81}$ and by Marenich et al ${ }^{82}$ using a statespecific polarizability model (SMSSP) coupled to a VEM description of the electrostatic effects. Cupellini et al. ${ }^{83}$ proposed instead an extension to electronic excitations of the dispersion-repulsion ground-state Hamiltonian proposed by Amovilli and Mennucci ${ }^{84}$ within the PCM formulation.

\subsubsection{Electrostatic shift method}

Another formulation of the environment effects on excitation energies has been proposed by Renger and coworkers ${ }^{17}$ explicitly for treating site energies of pigmentprotein complexes.

Such method comprises structure-based calculations of the free energy change of the PPC that occurs when the ground-state charge density of a given pigment is changed to that of the first excited state.

In its original formulation, also known as Poisson-Boltzmann/quantum chemical (PB/QC) approach, two steps were involved: ${ }^{85}$ in the first step, QC calculations are performed on pigments fully geometry-optimized in vacuo, ultimately resulting in atomic partial charges that describe the permanent charge distributions of ground and 
excited states and the transition densities. In the second step, these partial charges are used in a crystal-structure-based all-atom electrostatic calculation of the transition energy shift caused by transferring the pigment from a given reference solvent to the protein environment. We thus obtain the following expression for the site energy of pigment $m$ :

$$
E_{m}=E_{0}+\Delta E_{\text {ele }}^{(m)}
$$

where $E_{0}$ is a free adjustable parameter that represents the transition energy of the pigment in the reference solvent and $\Delta E_{e l e}{ }^{(m)}$ is calculated by means of classical electrostatics, where both protein and pigments are modeled by atomic partial charges embedded in a dielectric medium.

By applying the previously described nonequilibrium model, it is assumed that during excitation, the electrons but not the nuclei of the environment adapt to the newly established charge distribution of the pigment. As a consequence, the protonation pattern of the protein stays relaxed with respect to the ground state of the pigments, and the excited state of $m$ interacts with a nonequilibrium polarization of the protein that is only electronically relaxed. To obtain such a description $\Delta E_{e l e}^{(m)}$ is written as a full equilibrium term plus a correction, namely:

$$
\Delta E_{\text {ele }}^{(m)}=\Delta E_{e q}^{(m)}+\Delta E_{c o r r}^{(m)}
$$

where, for the equilibrium contribution, $\Delta E_{e q}{ }^{(m)}$, it is assumed that the charge distribution of the pigment in the excited state is in equilibrium with the total polarization of the environment (represented by the static dielectric constants of the protein and the solvent) and it can be determined by solving the linearized PoissonBoltzmann equation and a Monte Carlo procedure for the determination of protonation probabilities of titratable residues in the protein. For the nonequilibrium correction term, $\Delta E_{\text {corr }}^{(m)}$, one has to consider the interaction of the charge density difference between the excited and the ground state of the pigment $m$ with the surrounding dielectric. According to Marcus theory, a non-equilibrium free energy of an excited state may be obtained from the equilibrium free energy of the excited state and equilibrium free energies of two fictitious systems, which carry the charge density difference between excited and ground states of $m$ and are embedded in a dielectric with optical dielectric constant or static dielectric constant, respectively, In an effort to simplify this approach, an alternative method was successively developed, ${ }^{86}$ in which the polarization of the protein is described by an effective dielectric constant and a standard protonation pattern of the protein is assumed. In this charge density coupling (CDC)-method, the site energy shift $\Delta E_{\text {ele }}^{(m)}$ is obtained as 


$$
\Delta E_{\text {ele }}^{(m)}=\frac{1}{\varepsilon_{e f f}} \sum_{j} \Delta \phi^{(m)}\left(\mathbf{r}_{j}\right) q_{j}
$$

where $\Delta \phi^{(\mathrm{m})}$ is the difference in electrostatic potential between the excited and the ground state of pigment $m$. In the computational implementation, $\Delta \phi^{(\mathrm{m})}$ is represented in terms of partial charges of the ground and the excited state of the pigment $m$, as obtained from in vacuo quantum chemical calculations on the pigments and a fit of the electrostatic potential. The ground state partial charges $q_{\mathrm{j}}$ of the background, formed by the remaining atoms of the PPC, comprise those of the other pigments and those of the protein. The latter are taken from standard MM force fields. Since the protein contains residues with variable charge density, the titratable groups, the protonation pattern has to be determined before the site energy shifts can be calculated.

\subsubsection{QM description of the environment}

An alternative to classical formulations of the environment is provided by density-based embedding approaches, in which also the environmental degrees of freedom are calculated from first principles.

Examples in this field are the Fragment Molecular Orbital (FMO) method by Kitaura et al., ${ }^{87}$ the explicit polarization (X-Pol) theory by Gao et al ${ }^{88}$ and the Effective Fragment Potential (EFP) method by Gordon and co-workers. ${ }^{89}$

The main application of FMO is to compute very large molecular systems by dividing them into fragments and performing quantum-chemical calculations of fragments and their dimers, whereby the Coulomb field from the whole system is included. FMO has been applied to the photosynthetic reaction center of Blastochloris viridis: ${ }^{90}$ the protein complex of 20581 atoms and 77754 electrons was divided into 1398 fragments. The excited electronic states of the embedded chromophores were calculated by the CIS approach with the multilayer FMO method. Despite the structural symmetry of the system, asymmetric excitation energies were observed, especially on the bacteriopheophytin molecules. The asymmetry was attributed to electrostatic interaction with the surrounding protein.

Also X-Pol is a general fragment method for electronic structure calculations based on the partition of a condensed-phase or macromolecular system into subsystems ("fragments") to achieve computational efficiency. Moreover, X-Pol can also serve as a new-generation force field. In the X-Pol model the entire system is divided into molecular subunits, which can consist of one or more molecules, ions, ligands, cofactors, or amino acid residues. The key assumption in the X-Pol method is that the wave function of the entire system is approximated as a Hartree product of the antisymmetric wave functions of individual fragments. Then, the optimization of the total wave function can be reduced to the optimization of each fragment embedded in 
and polarized by the rest of the system; this reduces the computational cost to nearly linear scaling with respect to the number of fragments.

The effective fragment potential (EFP) method is a QM-based potential; ${ }^{89}$ it can be described as a nonempirical polarizable force field. The EFP method was originally developed specifically to describe aqueous solvent effects on biomolecular systems and chemical reaction mechanisms. Successively, a more general method has been developed to describe different kinds of environments. In the EFP the total energy of a system containing effective fragments and a QM part consists of the interactions between the effective fragments and the energy of the QM region in the field of the fragments. The former includes Coulomb, polarization, dispersion, and exchangerepulsion contributions. The QM-EFP interactions are computed using an electronic embedding approach: the Coulomb and polarization parts of the EFP potential contribute to the QM Hamiltonian via one-electron terms. The Coulomb potential due to fragments nuclear charges and electron density is represented by a multipole expansion, whereas the polarization operator describes the electrostatic field due to induced dipoles (thus, it depends on the polarizabilites of the fragments). The induced dipoles are iterated until self-consistency with each other and with the electronic wave function of the QM subsystem. Interface of EFP with EOM-CC and TDDFT has been developed to describe electronic excitations. ${ }^{91}$

An alternative to the aforementioned fragment-based schemes is the Subsystem Density Functional Theory $(\mathrm{sDFT})^{92}$ and the related Frozen Density Embedding (FDE) formalism $^{93}$ (see the reviews in Ref. ${ }^{94}$ and Ref. ${ }^{95}$ ).

Subsystem density-functional theory can be regarded as a hybrid method of KohnSham DFT for the systems $A$ and $E$. By minimizing the total energy expression with respect to the density of $A\left(\rho_{A}\right)$ given a fixed density of the environment $\left(\rho_{E}\right)$, one derives a set of Kohn-Sham-like equations for the optimal $\rho_{A}$, in which an effective embedding potential arises due to the presence of $\rho_{E}$. Since the same can also be done for system $E$, a scheme is derived in which systems $A$ and $E$ are iteratively polarized with respect to each other, until self-consistency. More in details, we rewrite eq 5.2 as:

$$
\begin{aligned}
& E^{s D F T}=E_{A}(K S)+E_{E}(K S)+V_{E A}\left(\rho_{A}, \rho_{E}\right) \\
& E_{X}(K S)=T_{s}\left(\left\{\phi_{X}\right\}\right)+J\left(\rho_{X}\right)+V_{X}^{n u c}\left(\rho_{X}\right)+E_{x c}\left(\rho_{X}\right) \quad(X=A, E) \\
& V_{E A}\left(\rho_{A}, \rho_{E}\right)=J\left(\rho_{A}, \rho_{E}\right)+V_{A}^{n u c}\left(\rho_{E}\right)+E_{x c}^{\text {nadd }}\left(\rho_{A}, \rho_{E}\right)+T^{\text {nadd }}\left(\rho_{A}, \rho_{E}\right)
\end{aligned}
$$

In eqs (19.2) $V_{\mathrm{X}}^{\text {nuc }}\left(\rho_{\mathrm{K}}\right)$ is the Coulomb attraction between the nuclei of system $X$ and the electron density of system $K, J$ is the Coulomb repulsion of a given electron density distribution or between the electron densities of the two systems, and $T_{S}$ is the kinetic energy as defined in the Kohn-Sham formalism, calculated from the Kohn-Sham 
orbitals, $\left\{\phi_{\mathrm{X}}\right\}$. The nonadditive $x c$ and kinetic functionals are defined as $F^{\text {nadd }}\left(\rho_{A}, \rho_{E}\right)=$ $F\left(\rho_{A}+\rho_{E}\right)-F\left(\rho_{A}\right)-F\left(\rho_{E}\right)$

By minimizing eq 18.2 with respect to the density $\rho_{A}$ (keeping $\rho_{E}$ fixed), one obtains a set of Kohn-Sham-like equations containing an effective embedding potential

$$
v_{e m b, A}(\mathbf{r})=v_{E}^{n u c}(\mathbf{r})+\int \frac{\rho_{E}\left(\mathbf{r}^{\prime}\right)}{\left|\mathbf{r}-\mathbf{r}^{\prime}\right|} d \mathbf{r}^{\prime}+\frac{\delta E_{x c}^{\text {nadd }}}{\delta \rho_{A}(\mathbf{r})}+\frac{\delta T_{x c}^{\text {nadd }}}{\delta \rho_{A}(\mathbf{r})}
$$

Here, $v_{E}^{\text {nuc }}$, is the Coulomb potential due to the nuclei of system $E$. A similar embedding potential, (with indices $A$ and $E$ interchanged) is obtained by minimizing the energy with respect to $\rho_{E}$. In practical applications, an iterative "freeze-and-thaw" scheme can be adopted in which systems $A$ and $E$ are mutually polarized until selfconsistency.

When the same formulation is extended to excited-state calculations, the environmental response can be included in the corresponding response-based subsystem TDDFT. The original TDDFT generalization of $\mathrm{FDE}^{96}$ leads to an effective kernel for excitation energies of the embedded system which is still, in principle, exact. However, this effective kernel would require calculating the full response of the environmental system, so that no overall savings in computational effort can be expected. The same generalization can be formulated in an approximate way that is straightforward to implement into TDDFT programs and that can be used to calculate local excitations of an embedded molecule. ${ }^{97}$ The approximation solves the response equations in terms of the orbital transitions of the embedded subsystem, assuming that the total density response can be approximated by the local response of the embedded-fragment density. The environmental effect enters the calculation in terms of the change in the orbitals and orbital energies, which is often dominant, and in terms of an additional contribution to the exchange-correlation kernel that stems from the embedding potential. In certain cases however, this approximated TDDFT formulation leads to qualitatively wrong results because couplings to the environmental response (which are neglected) become dominant,. This also means that excitation energy couplings between different subsystems cannot be described in this approximate formalism. More recently, it has been shown that it is possible to find an appropriate formalism that allows to include couplings between the embedded system and the environment still keeping the computational cost small compared to a supermolecular TDDFT calculation..$^{98}$

One drawback of FDE is its failure to describe fragments which are connected through covalent bonds such as in proteins. Attempts have been presented in which fragmented model structures have been used (see, e.g., Ref. ${ }^{99}$ ). In these schemes, some connecting groups are removed to split the protein into a few peptide chains. The remaining fragments are saturated to generate a number of environmental subsystems which are large, but still small enough to be treated in a conventional DFT calculation. Even if most of the protein is kept intact in this way, this procedure naturally forces one to alter the structure of the protein. An alternative way is the method of Molecular Fractionation 
with Conjugate Caps (MFCC) ${ }^{100}$ In the MFCC scheme, the protein is fragmented by cutting covalent bonds, but every fragment is saturated with capping groups in order to circumvent the problem of dangling bonds. To reconstruct the density of the supermolecule, combinations of the individual capping groups are also calculated and their density is subtracted from the sum of capped fragment densities. The MFCC scheme have been combined with the FDE method: the resulting approach was termed the 3-FDE method. ${ }^{101,102}$ Similar to regular FDE, an embedding potential is constructed from the densities of the individual subsystems (in this case the capped protein fragments). The electronic structure of each subsystem is then relaxed in the potential resulting from all the other fragments in so-called freeze-and-thaw cycles. ${ }^{103}$ The 3-FDE method has been recently extended to the calculation of excited-state properties and applied to the FMO protein. ${ }^{104}$

\section{ELECTRONIC COUPLINGS}

The second fundamental parameter needed, together with site energies, to construct an excitonic model, is the electronic coupling, namely the interaction between pair of electronic excitations localized on different units.

When the "bright" singlet excitations are considered, the coupling is dominated by the Coulomb interaction between transition densities, namely:

$$
V_{i j}^{C}=\int d \mathbf{r} \int d \mathbf{r}^{\prime} \rho_{i}^{T^{*}}\left(\mathbf{r}^{\prime}\right) \frac{1}{\left|\mathbf{r}-\mathbf{r}^{\prime}\right|} \rho_{j}^{T}(\mathbf{r})
$$

where $i$ and $j$ identify two excitations in two different units.

We recall that the transition density is a one-particle density matrix formed from the ground state and an excited-state wavefunctions

$$
\gamma_{e x c-g s}^{T}\left(\mathbf{r}, \mathbf{r}^{\prime}\right)=N \int \ldots \int \Psi_{e x c}^{*}\left(\mathbf{r}, \mathbf{r}_{2} \ldots \mathbf{r}_{N}\right) \Psi_{g s}\left(\mathbf{r}^{\prime}, \mathbf{r}_{2} \ldots \mathbf{r}_{N}\right) d \mathbf{r}_{2} d \mathbf{r}_{3} \ldots d \mathbf{r}_{N}
$$

and the transition density which determines the Coulomb coupling is the diagonal element of the matrix:

$$
\rho_{e x c-g s}^{T}(\mathbf{r})=\gamma_{e x c-g s}^{T}(\mathbf{r}, \mathbf{r})
$$

By definition, the transition density integrates to zero and it contains an equal amount of positive and negative elements with a zero total charge.

When the energy transfer involves one forbidden transition, it has been conventional to invoke mechanisms of electronic interaction other than Coulomb, such as electron exchange via orbital overlap as originally formulated by Dexter. ${ }^{105}$ The coupling 
between triplet excitations (Triplet energy transfer or TET) is an example. TET between a donor and an acceptor unit is overall a spin-allowed process; spin is conserved between the initial state ${ }^{3} \mathrm{D}^{* 1} \mathrm{~A}$ and the final state ${ }^{1} \mathrm{D}^{3} \mathrm{~A}^{*}$. As triplet-triplet energy transfer cannot be mediated by the Coulomb interaction because it is spin-forbidden, the coupling is generally written as a sum of exchange and overlap contributions:

$$
V_{i j}^{T E T}=V_{i j}^{e x c h}+V_{i j}^{o v l p}
$$

where the first term $V^{\text {exch }}$ is the (Dexter) exchange integral between the transition density matrices of the interacting units:

$$
V_{i j}^{\text {exch }}=-\iint d \mathbf{r}_{1} d \mathbf{r}_{2} \frac{\gamma_{i}^{T}\left(\mathbf{r}_{1}, \mathbf{r}_{2}\right) \gamma_{j}^{T}\left(\mathbf{r}_{1}, \mathbf{r}_{2}\right)}{\left|\mathbf{r}_{1}-\mathbf{r}_{2}\right|}
$$

whereas the second term can be estimated from the overlap of the transition densities.

Both terms of eq 4.3 require a nonzero superposition of donor and acceptor orbitals, and therefore present an exponential decay with the donor-acceptor separation.

The most straightforward way to get the coupling is by explicitly calculating the gap between the resulting excitonic states. In fact, it can be seen that the excitonic Hamiltonian of a two-state system, when diagonalized, leads exactly to the excitonic states $\mathrm{E}_{1}$ and $\mathrm{E}_{2}$ :

$$
\left(\begin{array}{cc}
\varepsilon_{i} & V \\
V & \varepsilon_{j}
\end{array}\right)_{\text {site }} \Leftrightarrow\left(\begin{array}{cc}
E_{1} & 0 \\
0 & E_{2}
\end{array}\right)_{e x c}
$$

where

$$
E_{1,2}=\frac{\varepsilon_{i}+\varepsilon_{j}}{2} \pm \sqrt{\left(\frac{\varepsilon_{i}-\varepsilon_{j}}{2}\right)^{2}+V^{2}}
$$

In the case of resonant excitations we have $\varepsilon_{i}=\varepsilon_{j}$ and the coupling becomes half of the energy gap.

The energy-gap scheme is a simple way to evaluate the coupling when the exact resonance conditions are satisfied and it is applicable to both singlet and triplet transfers. Its computational cost is that for the excited-state calculation of the full system. However, for general systems, the difficulty to search for the resonance condition increases rapidly with the size of the system and the application of the energygap scheme is, therefore, largely limited to small or highly symmetric systems. We also 
note that the environment by introducing an induced asymmetry makes the applicability of this approach even more limited.

Another direct approach is based on the construction of the eigenstates as symmetric and antisymmetric linear combinations of two diabatic states in which the excitation is localized on the donor (acceptor) chromophoric unit, respectively. Within such a scheme, the coupling is obtained through the expression

$$
V=\frac{H_{i j}-S_{i j}\left(H_{i i}+H_{j j}\right) / 2}{1-S_{i j}^{2}}
$$

where $H_{i j}$ are the off diagonal elements of the Hamiltonian matrix on the diabatic basis and $S_{i j}$ the overlap integrals.

For TET, the diabatic states can be modeled as the spin-localized states. ${ }^{106}$ Generally, the spin-localized diabatic states can be built by the broken-symmetry unrestricted Hartree-Fock (UHF) wavefunctions. ${ }^{107,108}$ The same formalism can be extended to unrestricted Kohn-Sham solutions. Moreover, still within DFT, the constrained density functional theory discussed above represents another possible way to obtain the diabatic states and it has been used to get TET couplings. ${ }^{109-112}$

For singlet energy transfer, this approach is much more computationally intensive as a singlet excited state typically involves several configurations. This means that one should consider many terms in configuration interactions, which rapidly increases the computational costs. Due to these limits, the direct evaluation of eq 8.3 is rarely applied in singlet energy transfer while most of the methods proposed so far try to solve the integral in eq 1.3. These methods are summarized in the following subsections.

\subsection{Singlet-singlet energy transfer (SET)}

\subsubsection{Methods based on transition densities}

In principle any quantum chemical method that gives access to transition densities can be used to compute the Coulomb component of the SET coupling through eq 1.3. As a matter of fact, in most applications the transition density is not directly used but an approximated form in terms of transition dipoles or transition atomic charges is instead adopted. As a result, the electronic coupling reduces to a classical dipole-dipole interaction energy or an electrostatic interaction among point charges.

The point dipole approximation (PDA) is the formulation most widely used, given that the values of the transition dipoles can in principle be extracted from absorption spectra of the involved pigments. The potential interaction between two dipoles can be found by considering the energy of one dipole in the field produced by the other one. The final expression is: 


$$
V_{i j}^{P D A}=\left|\mu_{i}\right|\left|\mu_{j}\right| \frac{\kappa}{R_{i j}^{3}}
$$

where $\left|\mu_{\mathrm{i}}\right|$ and $\left|\mu_{\mathrm{j}}\right|$ are the dipole lengths, $R_{i j}$ is the distance between the two dipoles and $\kappa$ accounts for the relative orientation between the dipoles (see scheme 1 ) and it is a pure geometrical factor defined as:

$$
\kappa=\hat{\boldsymbol{\mu}}_{i} \cdot \hat{\boldsymbol{\mu}}_{j}-3\left(\hat{\boldsymbol{\mu}}_{i} \cdot \hat{\mathbf{R}}_{i j}\right)\left(\hat{\boldsymbol{\mu}}_{j} \cdot \hat{\mathbf{R}}_{i j}\right)
$$

where the hat notation indicates the unit vector.

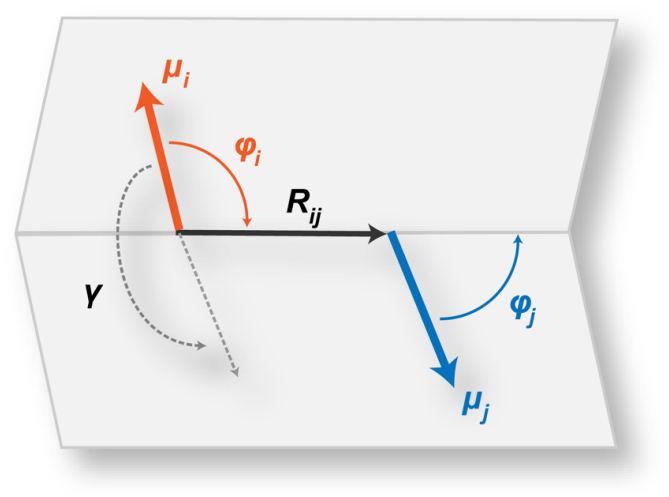

Scheme 1

The PDA formulation requires at least two fundamental data: i) orientation and magnitude of transition dipole moments, ii) the point of application of such dipoles, the so called "chromophore center". The magnitude of the transition dipole moment can be obtained experimentally from the absorption spectra. The experimental determination of the dipole orientation with respect to the molecule geometry is not easy and in general it can be obtained by QM calculations or using the symmetry rules. A mixed approach is also often used by using the QM dipole orientation and rescaling its magnitude on the basis of the experimental values.

The main limit of PDA is that the dipole approximation works well only for interchromophoric distances much larger than the dimensions of the chromophores. At short distances PDA may give largely over-estimated couplings. ${ }^{13}$ Moreover, when the two transition dipoles are close to perpendicular, the geometrical factor, $\kappa$, approaches zero and consequentially also the PDA coupling goes to zero, while in the case of the full transition densities it does not happen. On the other hand, in the range of reliability of the PDA approximation, eq 9.3 is very useful as it gives a simple interpretation of a coupling in terms of dipole strengths, their relative orientation and the distance.

A more cost-effective and accessible method of modeling the Coulomb coupling is to decompose the transition density into point charges localized on the atoms of the 
involved pigments; this approach has been often indicated as "transition monopole approximation" (TMA) method. ${ }^{114-116}$

Different approaches exist to calculate atomic charges on the basis of the density or transition density. One method, similar to the Mulliken population analysis, uses the density to obtain populations centered on the atomic nuclei and converts these populations into charges, called transition charges. ${ }^{117,118}$ The density can be obtained by a variety of QM levels, either semiempirical or ab initio. Atomic charges can be obtained by other methods besides population analysis. A quite popular method, which has been used extensively to determine charges for the ground state, involves a fitting of the electrostatic potential around the molecule. This method has been extended to transition charges, and is also known as TrEsp (transition charge from electrostatic potential) method. ${ }^{119}$

Within the transition monopole approximation, the Coulomb coupling reduces to:

$$
V_{i j}^{T r E s p}=\sum_{K, L} \frac{q_{k}(i) q_{L}(j)}{\left|\mathbf{R}_{K}-\mathbf{R}_{L}\right|}
$$

where $K$ and $L$ run on the atoms of the chromophoric units involved in the excitation $i$ and $j$, respectively, and $\mathbf{R}_{X}$ are the corresponding spatial positions.

Regardless of the approximate nature of the transition charges, they can be very useful in studies of aggregates, where other methods are too expensive.

More general methods have been proposed which use the "full" definition reported in eq 1.3. These methods mainly differ in the numerical solution used to calculate the volume integral. Earlier works made use of semi-empirical Hamiltonians..$^{33,34,120,121}$ With ab initio methods, a full account for the Coulomb coupling was originally calculated by first recording the transition density in three-dimensional (3D) grids, followed by a numerical integration. This approach, known as Transition Density Cube (TDC), ${ }^{122,123}$ reformulates the integral in eq 1.3 by expressing the transition density as an array of finite-sized volume elements (the transition cubes)

$$
M_{i}(\mathbf{r})=\int_{z}^{z+\delta z} \int_{y}^{y+\delta y} \int_{x}^{x+\delta x} \rho_{i}^{T}(\mathbf{r}) d x d y d z
$$

where $\mathrm{V}_{\delta}\left(\mathrm{V}_{\delta}=\delta \mathrm{x} \delta \mathrm{y} \delta \mathrm{z}\right)$ is the element volume needed as a practical means of converting charge density per unit volume into charge density per element. Finally, the Coulomb interactions between all the elements of each cube are summed to get the final coupling:

$$
V_{i j}^{T D C}=\sum_{k, l} \frac{M_{i}(k) M_{j}(l)}{\left|\mathbf{r}_{k}-\mathbf{r}_{l}\right|}
$$

The accuracy of eq 13.3 is limited by the number of elements in the TDC, the size of 
each element, and the quality of the quantum chemical wavefunctions. Particularly, the finite size of density cubes results in an underestimation of charge-penetration effects due to the exponential decay and diffuse character of the transition charge densities. Another consequence of the grid is the problem of residual charge. That is, the sum of the charge over all cube elements is not zero, as it should be. This residual charge can significantly affect the calculated coupling because it provides spurious charge-charge and charge-dipole interactions between the transition densities. To compensate for this residual charge $\mathrm{q}^{\mathrm{R}}$ in a TDC with $\mathrm{N}$ elements, a quantity $\mathrm{q}^{\mathrm{R}} / \mathrm{N}$ is subtracted from each element in the TDC, such that the residual charge is largely reduced. Upon evaluating eq 13.3, one must also remove singularities that arise when cube elements of the donor TDC overlap with those of the acceptor TDC. This can be amended by imposing appropriate cutoff distances, but such a procedure clearly affects the accuracy. The TDC method is also quite demanding computationally and becomes impractical for large chromophores because the number of grid points can easily exceed hundreds of thousands. This difficulty is amended in the TrESP scheme where the number of distributed sites is equal to the number of atoms. However, this inevitably leads to a loss of accuracy, which is particularly pronounced at smaller interchromophore distances. ${ }^{124,125}$ In particular, it was shown that the TrESP method can substantially underestimate the couplings, and the values obtained by using TDC and TrESP schemes can differ substantially. Recently, an extension of TrESP protocol was proposed ${ }^{26}$ in which the atomic transition charges (C) are augmented with the atomic dipoles (D) and quadrupoles $(\mathrm{Q})$ derived from electrostatic potential fitting (TrESP-CDQ). The TrESPCDQ approach has shown improvements with respect to the original TrESP method; however, its accuracy still strongly depends on the selection of the grid to fit the transition multipoles and the fitting procedure itself. In order to search for a more general solution, independent of the fitting procedure, a new scheme based on the distributed multipolar expansion has been proposed. ${ }^{127}$ Also this scheme (called TrCAMM) is based on the distributed multipolar expansion of the one-particle transition densities obtained from ab initio or TD-DFT calculations, but it adopts the cumulative atomic multipole moments scheme (CAMM) ${ }^{128}$ up to hexadecapole. 


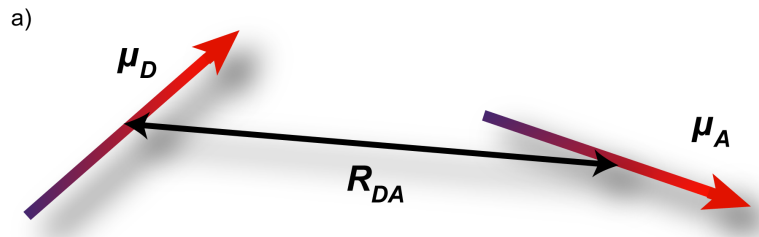

b)

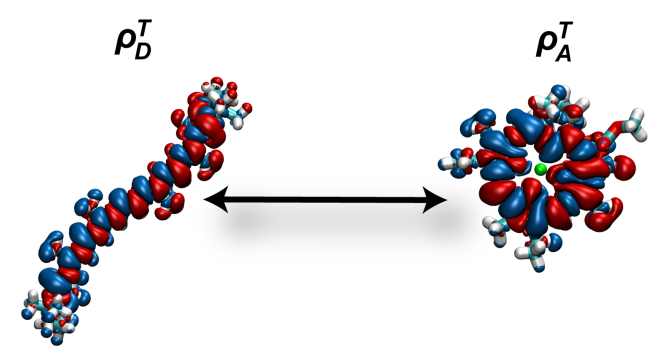

c)

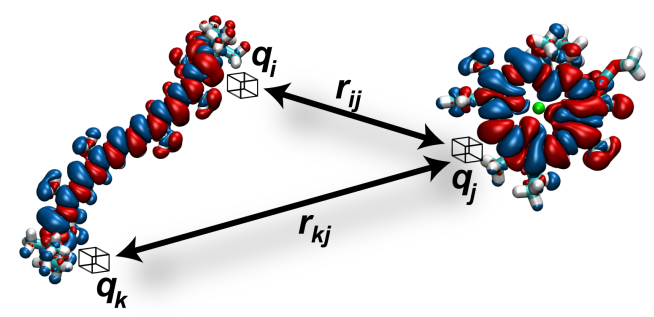

Figure 1. Graphical representation of transition densities obtained for a chlorophyll and a carotenoid molecule and different schemes used to compute their interaction. a) Point dipole approximation (eq 9.3). b) Full interaction between transition densities (eq 1.3). c) Methods based on the discretization of the transition density over atomic centers (TrEsp method, eq 11.3) or over grid points (TDC method, eq 13.3).

More accurate and numerical stable formulations have been proposed by directly solving the integral (1.3) through the efficient grid-based integration methods used in DFT $^{129}$ without the need of introducing any multipolar expansion neither a cube grid, as illustrated in Figure 1. This method has been applied to many different QM methods, ${ }^{130}$ from CIS and TDDFT to CASSCF and Coupled Cluster descriptions. ${ }^{63}$ Moreover it has been coupled to polarizable classical methods to include environment effects (see below).

\subsubsection{Environment effects}

The presence of an environment affects the Coulomb coupling in two ways. The first one is exactly the same leading to changes in the site energies: namely the environment can change both the geometrical and the electronic structure of the interacting chromophores thus modifying their transition properties, i.e. transition dipoles and transition densities. These changes will be reflected in different couplings. Secondly, if the environment can polarize, the direct Coulomb interaction between excitations in 
different chromophores will be "screened" by the environment. These screening effects were traditionally taken into account in the PDA coupling in terms of an effective dielectric constant through a $1 / \varepsilon_{\text {eff }}$ scaling term, an approximation already present in the early formulation due to Förster. ${ }^{131}$ The same scaling factor has also been adopted in TrEsp and TDC approaches to scale their respective electrostatic formulations of the coupling.

Attempts to improve the Förster scaling term still keeping its simplicity have exploited the concept of local field factors.

To explain this concept we have to consider an average electric field $\mathbf{E}$ representing the incident light in the medium which acts on the molecular transition moment $\mu$. The resulting interaction $\boldsymbol{\mu} \cdot \mathbf{E}$ may be rewritten as $f \boldsymbol{\mu}_{0} \cdot \mathbf{E}$ where $\mu_{0}$ is the transition dipole in vacuo. This interaction can be thought in two ways, as either $\left(f \boldsymbol{\mu}_{0}\right) \cdot \mathbf{E}$ or $\boldsymbol{\mu}_{0} \cdot(f \mathbf{E})$. In the former case, one has the transition moment $\boldsymbol{\mu}=f \boldsymbol{\mu}_{0}$ that interacts with the average field. In the latter case, one has the vacuum transition moment $\mu_{0}$ interacting with an effective field $\mathbf{E}_{\text {eff }}=f \mathbf{E}$, evoking the more classical view that $f$ is an "effective field" factor.

The concept of local field was established by Lorentz at the beginning of the last century. He showed that for atoms or nonpolar molecules with well-localized bound electrons the local field factor $f_{l}$ can be written as:

$$
\mathbf{E}_{l o c}=f_{l} \mathbf{E}=\frac{2+\varepsilon}{3} \mathbf{E}
$$

The validity of this approach rests on the hypothesis that the polarization within the dense medium is uniform.

A refinement to the Lorentz model is due to Onsager. In this framework, the local field correction has to be evaluated by considering the field inside an empty spherical cavity when subject to a homogeneous external field (often this contribution is called the "cavity field"). In this case, we have

$$
f_{O}=\frac{3 \varepsilon}{2 \varepsilon+1}
$$

In the context of screening effects, these factors are introduced to describe the change in effective dipole strengths of the chromophores due to the boundary with the surrounding dielectric. ${ }^{131}$ 


\subsubsection{QM/classical models}

In hybrid $\mathrm{QM} / \mathrm{CD}$ formulations the screening due to the environment can be selfconsistently obtained by an explicit "environment" term to the Coulomb coupling ${ }^{129,132}$ which naturally comes out when the proper effective Hamiltonian is used (see eqs 6.27.2). In particularly, by using the PCM framework such term can be written as ${ }^{129}$

$$
V_{i j}^{P C M}=\sum_{k} \int d \mathbf{r}^{\prime} \rho_{i}^{T^{*}}\left(\mathbf{r}^{\prime}\right) \frac{1}{\left|\mathbf{r}^{\prime}-\mathbf{s}_{k}\right|} q_{k}^{P C M}\left(\rho_{j}^{T}\right) d \mathbf{r}^{\prime}
$$

where $q^{\mathrm{PCM}}$ are the charges induced on the cavity surface by the electronic transition in the chromophore $\mathrm{j}$. These charges, which are determined assuming a nonequilibrium response, allow describing a screening that depends not only on the electronic transitions involved but also on the inter-pigment distance and orientation.

Within this framework, it is still possible to dissect the screening effect by defining an "effective" screening factor and a related effective permittivity as: ${ }^{133,134}$

$$
s=\frac{1}{\varepsilon_{\text {eff }}}=\frac{V_{s}+V_{\text {expl }}}{V_{s}}
$$

An analysis over 100 chromophore pairs, including chlorophylls, bilins and carotenoids, taken from structural models of photosynthetic pigment-protein complexes, unveiled a strong exponential attenuation of screening effects at distances below $20 \AA$, which turns to be the range of distances where relevant interactions in photosynthetic complexes occur. This behavior occurs when the chromophores approach each other, thus excluding the environment (solvent or protein residues) from the intermolecular region. On the other hand, at large separations screening factors approach the values predicted by Förster. The results were fitted with a distance-dependent screening function, averaged over multiple chromophores, shapes and orientations, the resulting expression is:

$$
s=A \exp (-\beta R)+s_{0}
$$

where the pre-exponential factor is $A=2.68$, the attenuation factor is $\beta=0.27 \AA^{-1}$, and $s_{0}=0.54$ is the asymptotic value of $s$ at large distances. These results were obtained using a $n^{2}=2$ value for the optical dielectric permittivity of the environment. In addition, static dielectric properties were simulated using a permittivity $\varepsilon=15$. 
Renger and co-workers ${ }^{17}$ have also examined this issue in detail for several pigmentprotein complexes, including the FMO protein, photosystem I (PSI) and the lightharvesting complex II (LHCII), using the model outlined in section 2.2.2.

Two formulations have been used; in the simplest one the TrEsp expression of the coupling (eq 11.3) is used in combination of a fixed scaling factor $f$ to implicitly take into account screening and local field effects by the dielectric environment. In the second, and more refined, formulation, also known as Poisson-TrEsp method, the influence of the dielectric environment on the excitonic coupling is modelled explicitly. A Poisson equation is solved for the potential $\phi_{m}(\mathbf{r})$ of the transition charges of pigment $\mathrm{m}$ placed in a molecule-shaped cavity that is surrounded by a homogeneous dielectric with dielectric constant $\varepsilon$ equal to the square of the refractive index, which represents the optical polarizability of the protein and solvent environments. The excitonic coupling of pigment $\mathrm{m}$ with pigment $\mathrm{n}$ is then obtained as $^{86}$

$$
V_{m n}=\sum_{J} \phi_{m}\left(R_{J}\right) q_{J}^{(n)}
$$

where the transition charges $\mathrm{q}(\mathrm{m})(0,1)$ of the pigments are obtained as described above for the TrEsp method (see section 3.1.1).

By comparing Poisson-TrEsp couplings obtained with $\varepsilon=2$ and $\varepsilon=1$ (corresponding to unscaled TrEsp) it was found that the screening and local field corrections of the Coulomb coupling can be well approximated by a constant factor $f$, which varies between 0.7 and 0.8 for the investigated complexes. However, this strategy implies that larger errors are introduced in small couplings. As the authors point out, this leads to negligible effects on predicted spectra, whereas it may have larger implications regarding energy transfer rates.

If now we substitute the continuum description of the environment with an atomistic one using the polarizable $\mathrm{MM}$ approach described in section 2.2.1, the explicit contribution to the coupling due to the intervening effect of the polarizable environment becomes: ${ }^{135}$

$$
V_{i j}^{M M P o l}=-\sum_{l} \int d \mathbf{r}^{\prime} \rho_{i}^{T^{*}}\left(\mathbf{r}^{\prime}\right) \frac{\left(\mathbf{r}^{\prime}-\mathbf{r}_{l}\right)}{\left|\mathbf{r}^{\prime}-\mathbf{r}_{l}\right|^{3}} \cdot \mu_{l}^{M M p o l}\left(\rho_{j}^{T}\right) d \mathbf{r}^{\prime}
$$

where the induced dipoles are now determined by the transition density corresponding to the excitation $\mathrm{j}$.

The two polarizable formulations, QM/PCM and QM/MMPol, have been shown to give very similar results not only for homogeneous environments like common solvents but also for more complex matrices such as proteins. ${ }^{135,136}$ In these cases, however, the similarity is true at an average level while the two models can lead to quite different results when different pairs of pigments placed in different positions within the protein matrix are analyzed: due to molecular differences in the local environment around each 
pigment (which obviously imply differences in the polarizability) the $\mathrm{V}^{M M P o l}$ term can change significantly from one pair to the other. ${ }^{136-139}$

\subsubsection{QM/QM descriptions}

Another approach to include environment effects in the coupling is through the FDE described in section 2.2.3.

As reported before, FDE has been successfully applied to describe excitations in large multichromophoric systems by using a two-step approach. ${ }^{98,140,141}$ First, the uncoupled FDE excitation energies are calculated; i.e., local excitations of all constituent fragments are obtained, embedded in an environment formed by all other fragments. These calculations employ the ground-state FDE embedding formalism discussed above in which the environmental density is obtained as a sum of all other fragments' densities. To obtain optimum subsystem densities, freeze-and-thaw cycles are iteratively applied to all subsystems, so that effectively a variational subsystem density functional theory treatment is performed. In a second step, delocalized excited states of the entire aggregate are calculated by coupling these local excitations. A typical criterion that was used in the original implementation to determine states that have to be included was the energy difference between a particular excited state and the reference states, since highlying excited states only have a minor effect on the excitonic states. When modeling solvent effects, however, the situation is somewhat more complicated. ${ }^{142}$ The solvent screening can, in a linear-response TDDFT framework, be understood as a cumulative effect caused by many excited states of the solvent system. Consequently, a very large number of excited states would have to be included, which considerably increases the effort for FDE calculations.

To reduce such a computational cost, a two-step strategy has been introduced. ${ }^{142}$ In order to achieve a reasonable representation of the solvent response, one first determines how many excited states are necessary to represent the (isotropic) polarizability of a solvent molecule to a good accuracy in terms of the sum-over-states (SOS) expression. Once these excitations are calculated for all (embedded) solvent molecules, they are sorted according to their contribution to the SOS polarizability expression in descending order. From this list of states, one chooses the first $k$ states, where the number $k$ is determined in such a way that the cumulative contribution of these states is larger than a preselected threshold percentage of the full SOS polarizability.

Another approximation that was examined consists in the neglect of inter-solvent couplings, so that solvent excited states are only coupled to the dye molecules' excited states. In that study, a rather good agreement was found between SET couplings and environment screening effects obtained either from FDE and QM/MMpol calculations in a solvated perylene diimide dimer, with discrepancies between the two approaches being on the order 6 to $10 \%{ }^{142}$ 


\subsubsection{Bridge effects, through-bond and charge transfer}

In the previous analysis of the coupling, we have implicitly assumed that the two interacting units were not chemically bonded. In many important applications of EET, instead, covalently linked arrays are used where a semirigid linker is generally introduced between the donor and acceptor chromophores to keep a well-defined and rigid structure and maintain some properties of the isolated chromophores, so that the resulting complex has predictable characteristics, while imparting efficient electronic communication channels among the chromophores

Among possible effects arising from the presence of a bridge between the chromophores, one is represented by the so-called "superexchange" interaction operating beyond actual orbital overlap region and usually thought to be mediated by electronic coupling of the interchromophore bridge orbitals. ${ }^{143}$

A thorough theoretical analysis of such a mechanism has been done by McConnell ${ }^{144}$ for electron-transfer phenomena showing that intramolecular transfer mediated through a $\sigma$-bonded bridge should decrease exponentially with the bridge length. Successively, the superexchange mechanism (often indicated as simply through-bond mechanism) has been extended to EET using a perturbative approach. Within this framework, an effective coupling is introduced which accounts for the perturbation of the donor wave function due to the coupling to the bridge states: ${ }^{145}$

$$
V=V_{D A}+V_{D-B-A}
$$

with

$$
V_{D-B-A}=\sum_{i} \frac{V_{D-B^{i}} V_{A-B^{i}}}{\left(\Delta E_{D}-\Delta E_{B^{i}}\right)}
$$

where $V_{x: B i}$ denotes the electronic coupling between the donor $(X=D)$ or acceptor $(X=$ $A)$ transition, respectively, and the bridge $i$ th transition with the vertical electronic transition energy $\Delta E_{B} ; \Delta E_{D}$ is the vertical electronic emission energy of the donor.

A further aspect to consider in donor-bridge-acceptor systems, besides superexchange, is the charge-transfer (CT) contribution. Such a term has been introduced in the QM modelization by Harcourt et al. ${ }^{146}$ by using a configuration interaction approach including locally excited donor and acceptor configurations as well as "bridging" ionic configurations. The important issue introduced in such a model is a new orbital overlapdependent interaction (indicated as through-configuration interaction) which involves successive, virtual, one-electron transfers mediated via the ionic bridging configurations. The original version of the model starts by defining a reduced chromophore-localized basis set. Specifically, the HOMO and LUMO orbitals localized on each chromophore are included. For two equivalent chromophores, this four orbital 
basis leads to four singly excited configurations: $\psi_{1}=\left|C^{*} C\right\rangle, \psi_{4}=\left|C C^{*}\right\rangle, \psi_{2}=\left|C^{+} C^{-}\right\rangle$, and $\psi_{3}=\left|C^{-} C^{+}\right\rangle$. The first two of these are the "covalent" configurations, which are usually considered in the theory of excitonic states. The last two are "ionic" or chargetransfer configurations, and they lead new contributions to the coupling, which can be rewritten as:

$$
T_{R P} \approx T_{14}-\left(T_{12} T_{24}+T_{13} T_{34}\right) / A
$$

where $A$ is the energy difference between 2 and 1 (or 3 and 4) and $T_{i j}=\left\langle\psi_{i}\left|H-E_{1}\right| \psi_{j}\right\rangle$.

Examination of eq 23.3 shows that the primary terms which promote donor-acceptor EET are: (a) A direct exciton resonance interaction, $\mathrm{T}_{14}$, which is associated with a two-electron transfer between the donor and acceptor configurations 1 and 4. (b) Through-configuration exciton resonance interactions, $-\mathrm{T}_{12} \mathrm{~T}_{24} / A$ and $-\mathrm{T}_{13} \mathrm{~T}_{34} / A$, which are associated with two equivalent one-electron transfer processes mediated via the ionic bridging configurations, namely 1-2-4 and 1-3-4. The same authors also showed that the direct interaction is primarily Coulombic (even if it also contains exchange integrals corresponding to the terms seen in the Dexter formulation), whereas the throughconfiguration interaction is associated with overlap-dependent terms.

The Harcourt model, in its generalized version allowing for larger basis sets not limited to the HOMO-LUMO on each chromophore, and for nonequivalent chromophores, has been also extended to include solvent effects ${ }^{47}$ using the PCM approach described in section 2.2.1. Within this framework, the requirement needed to incorporate solvent effects in the through-configuration interaction term is automatically fulfilled by using the effective Hamiltonian expression defined in eq 7.2. The only specificity to take into account is that we have to know the CI density matrix of the state of interest. Such nonlinear character can be solved through an iterative procedure; at each iteration the environment-induced component of the effective Hamiltonian is computed by using the environment response obtained with the first-order density matrix of the preceding step. We note that this procedure is valid for ground and excited states fully equilibrated with the solvent but, by introducing some refinements, the inclusion of the nonequilibrium can be obtained.

Another possible effect of the bridge is that its polarizability can explicitly affect the Coulomb interaction between the D/A moieties ${ }^{148-152}$ This effect can be accounted for both using QM descriptions of the bridge or polarizable embedding schemes in QM/MM formulations. In the latter case the bridge is treated as a set of polarizable MM sites and additional polarization effects due to the external environment can be also added through a continuum description. ${ }^{152}$ 


\subsubsection{Beyond the Coulomb coupling: the diabatization strategy}

In singlet energy transfer, the Coulomb coupling should be complemented with two (minor) terms representing the Dexter exchange coupling that accounts for the indistinguishability of the electrons in many-electron wave functions, and a term arising from the overlap of donor-acceptor electronic transition densities:

$$
V=V_{\text {coul }}+V_{\text {exc }}+V_{\text {over }}
$$

where $V_{\text {exc }}$ and $V_{\text {over }}$ have the same meaning discussed for triplet transfer. We expect that in the case of singlet energy transfer, the non Coulomb terms become important for short-range transfers.

A proposal to account for non Coulomb terms but still maintaining a separation of the chromophoric units as independent QM fragments was made by Hsu et al. within the TDDFT description of a pair of donor $D$ and acceptor $A$ molecules having a resonant excitation frequency $\omega_{0}$ when there is no interaction between them. ${ }^{132}$ The final expressions state:

$$
\begin{aligned}
& V_{D A}^{x c}=\int d \mathbf{r} \int d \mathbf{r}^{\prime} \rho_{D}^{T^{*}}\left(\mathbf{r}^{\prime}\right) g_{x c}\left(\mathbf{r}^{\prime}, \mathbf{r} ; \omega_{0}\right) \rho_{A}^{T}(\mathbf{r}) \\
& V_{D A}^{\text {over }}=-\omega_{0} \int d \mathbf{r} \rho_{D}^{T^{*}}(\mathbf{r}) \rho_{A}^{T}(\mathbf{r})
\end{aligned}
$$

where $g_{x c}$ is the exchange-correlation kernel of the selected DFT functional.

A more general approach to go beyond the Coulomb approximation to the coupling is by introducing a picture in terms of diabatic states. ${ }^{153}$ The main problem is that there is not a unique prescription for defining "exactly" the diabatic states, ${ }^{154}$ i.e. the initial and final electronic states of a physical system undergoing electron transfer or excitation energy transfer. The goal is to determine the adiabatic-diabatic transformation matrix by imposing some reasonable criterion generally based on chemical intuition. Different diabatization schemes have been successfully derived to estimate the total excitonic/electronic couplings (short- and long-range contributions). These schemes can be mainly divided into two groups: those based on the wave-function and those based on molecular properties. Diabatic states can also be produced directly using constraints on the electronic density such as in the previously recalled C-DFT approach. ${ }^{155,156}$

In the context of electron or excitation energy transfer, the diabatization schemes based on molecular properties have become more popular. The main advantage of these diabatization schemes is that they can, in principle, be used independently of the level of theory. A particularly frequent choice among these diabatization schemes is the 
Generalized Mulliken-Hush (GMH) method, ${ }^{157}$ which is based on its predecessor method (Mulliken-Hush model) and identifies the transformation that diagonalizes the adiabatic dipole moment matrix. When the transformation is applied to the adiabatic (diagonal) Hamiltonian, the diabatic Hamiltonian is obtained and the electronic couplings correspond to its off diagonal elements.

Still within the context of molecular property-based diabatic transformations it is worth here mentioning the fragment excitation difference (FED) scheme. ${ }^{158}$ The FED is a generalization to energy transfer of the "fragment charge difference" (FCD) method developed by Voityuk et al. ${ }^{159}$ for determining electron transfer couplings.

In a single excitation model, the difference density between two states in the molecular orbital basis contains a negative occupied-occupied block and a positive virtual-virtual block, the absolute value of the former representing the detachment, or the "hole" population, and the latter the attachment, or the "electron" population: ${ }^{160}$

$$
\Delta \rho(\mathbf{r})=\rho^{a t t}(\mathbf{r})-\rho^{d e t}(\mathbf{r})
$$

The state-to-state transition density, $\rho_{m n}^{e x}$, can be also expressed in terms of the molecular orbital basis and it is similarly composed of an occupied-occupied and a virtual-virtual block. We can thus define the hole transition density as the negative value of the occupied-occupied block, while the electron transition density is defined as the virtual-virtual block:

$$
\rho_{m n}^{e x}(\mathbf{r})=\rho_{m n}^{h o l e}(\mathbf{r})+\rho_{m n}^{e l e}(\mathbf{r})=\rho_{m n}^{\mathrm{det}}(\mathbf{r})+\rho_{m n}^{a t t}(\mathbf{r})
$$

Starting from these matrices it is possible to define the FED matrix $\Delta \boldsymbol{x}$ as a measure of the excitation localization,

$$
\Delta \mathbf{x}=\left(\begin{array}{cc}
\Delta x_{m m} & \Delta x_{m n} \\
\Delta x_{m n} & \Delta x_{n n}
\end{array}\right)
$$

where its matrix elements $\Delta x_{m n}$ are defined as:

$$
\Delta x_{m n}=\int_{\mathbf{r} \in D} \rho_{m n}^{e x}(\mathbf{r}) d \mathbf{r}-\int_{\mathbf{r} \in A} \rho_{m n}^{e x}(\mathbf{r}) d \mathbf{r}
$$

The excitation-localized states are thus the states that diagonalize the FED matrix while the coupling can be obtained as:

$$
V_{m n}^{F E D}=\frac{\left(E_{n}-E_{m}\right)\left|\Delta x_{m n}\right|}{\sqrt{\left(\Delta x_{m m}-\Delta x_{n n}\right)^{2}+4\left(\Delta x_{m n}\right)^{2}}}
$$


In the symmetrical situation (identical donor and acceptor), the coupling becomes onehalf of the energy gap $\left(E_{m}-E_{n}\right)$, as expected.

Since eigenstate properties are used, the resulting EET coupling will reflect the full coupling under the level of the theory employed for the eigenstates. No further constrains on the excitation are required, and FED coupling can be used for general systems, regardless of their symmetry. The FED scheme has been formulated within CIS and the Tamm-Dancoff framework of TDDFT for a two-state model.

A more general approach which allows one to generate diabatic states applicable both to ET and EET processes, has been proposed by Subotnik and co-workers: ${ }^{161,162}$ the diabatization routine distributes charge or exciton automatically and does not require to define donor and acceptor fragments. The method can be applied to multi-state systems with any wavefunction type. The use of this method to EET may be restricted, however, for some molecular systems by the difficulty of localizing transition densities. A general Fragment Transition Density (FTD) approach has been introduced by Voityuk ${ }^{163,164}$ to treat multi-chromophore systems. The FTD scheme has no limitation on the type of the excited-state wavefunction and can be applied in combination with different quantum mechanical methods.

Very recently, a novel and general diabatization scheme, based on dipole and quadrupole moments (called the DQ method), has been developed by Hoyer et al. to compute electronic couplings between different electronic states. ${ }^{165}$

\subsection{Triplet-triplet energy transfer (TET)}

As reported in eq 4.3, the triplet coupling may be seen as a sum of exchange and overlap contributions. Both terms require a nonzero superposition of donor and acceptor orbitals, and therefore present an exponential decay with the donor-acceptor separation. However the interaction is expected also to be highly sensitive to molecular shape, size, and orientation.

Early experimental studies by Closs and coworkers ${ }^{166}$ on intramolecular triplet transfer suggested a close correspondence between TET and electron transfer. In this picture, TET is a double electron transfer involving LUMOs and HOMOs: as a result, the probability of TET becomes proportional to the product of the probabilities of the electron transfer between the LUMOs (ET), and the electron transfer between the HOMOs (hole transfer, HT) of donor and acceptor:

$$
k_{T E T}=C k_{E T} k_{H T}
$$

where the constant $C$ depends on the particular system under study. The same experiments, in addition, revealed an exponential attenuation of TET couplings with donor-acceptor separation consistent with the superexchange model originally 
developed by McConnell for electron transfer. The mediation of charge-transfer configurations in determining the triplet transfer orbital overlap-dependent interactions was later confirmed by various theoretical analyses. ${ }^{4}$

\subsubsection{Fragment spin density (FSD)}

The experimentally observed and theoretically proved parallelism between TET and ET has allowed recent theoretical and computational work to calculate energy transfer rates via the Marcus formula ${ }^{167}$ for electron transfer rates. ${ }^{168-170}$ This has been made possible by realizing that diabatic states can be calculated for TET just as for ET and one can use a localized diabatization scheme to determine the parameters that enter into the Marcus rate equation.

An example of this strategy is the fragment spin difference (FSD) scheme ${ }^{171}$ which can be seen as the generalization of the FED scheme described in section 3.1.4 to triplet states. In FED, a $2 \times 2$ excitation difference matrix is calculated for the two eigenstates of interest, and the diabatic states are defined as the linear combination of the two states that give rise to the maximum difference excitation population. For TET, spin population can be used to replace the excitation population in FED, which is the difference between one-electron $\alpha$ and $\beta$ electron density functions,

$$
\sigma_{m n}(\mathbf{r})=\rho_{m n}^{\alpha}(\mathbf{r})-\rho_{m n}^{\beta}(\mathbf{r})
$$

where $m=n$ denotes a spin density function of the $n$th excited state and $m \neq n$ denotes a state-to-state transition spin density function between the $n$th and the $m$ th states.

Following the FED strategy, the diabatic initial and final states are defined as the states that maximize the spin difference between donor and acceptor, and can be computed as the eigenvectors of the operator $\Delta \mathrm{s}$ :

$$
\Delta s_{m n}=\int_{\mathbf{r} \in D} \sigma_{m n}(\mathbf{r}) d \mathbf{r}-\int_{\mathbf{r} \in A} \sigma_{m n}(\mathbf{r}) d \mathbf{r}
$$

In order to calculate the FSD matrix element $\Delta \mathbf{s}_{m n}$, the Mulliken population analysis has been originally used for the densities together with a sum over the atomic contributions in each fragment.

Once the diabatic states are found, one writes the electronic Hamiltonian in the diabatic basis, where the off-diagonal element represents the triplet coupling:

$$
V_{m n}^{F S D}=\frac{\left(E_{n}-E_{m}\right) \Delta s_{m n}}{\sqrt{\left(\Delta s_{m m}-\Delta s_{n n}\right)^{2}+4\left(\Delta s_{m n}\right)^{2}}}
$$


From eq 35.3 it comes out clearly that the FSD coupling becomes a half-energy gap when the system is symmetric.

For a spin-restricted case, the FSD coupling is essentially a generalization of FED to triplet states. However, for spin-unrestricted cases, the reference state may have a spin population, and therefore the FED scheme does not apply. The FSD scheme has also shown that for many cases, the coupling values are much larger than the conventional exchange integral, therefore indicating that the overlap contribution is important in TET coupling.

The FSD and the FED schemes have been applied to study triplet energy transfer processes in both natural light-harvesting complexes, ${ }^{172}$ DNA $^{173}$ and artificial systems. ${ }^{10,174}$ More recently the FSD scheme has been generalized to calculate the singlet fission coupling. ${ }^{175}$

\subsubsection{Environment effects}

For TET, the inclusion of environment effects is more involved than in SET as the previous formulations based on the response of the environment to the transition density, here cannot be applied. In fact there is no such environment effect because there is no Coulomb coupling. What is expected is that the intervening medium allows a better electron tunneling, and the one-electron orbitals are with longer exponential penetration. In this case, the environment should be explicitly considered in the quantum chemical calculations. In this way, the intervening solvent can be treated as a nonresonant bridge and the superexchange theory can be applied.

This picture has been confirmed for the role of water in the TET process between chlorophyll and peridinin in PCP complex by a combined spectroscopic-computational investigation. ${ }^{176}$ Another QM/MD study ${ }^{177}$ based on FED has shown that an intervening solvent can modify the distance dependence of TET in a stacked perylene dimer. By computing electronic coupling values by adding solvent molecules in a sequential manner, the authors were able to dissect the effect of individual solvent molecules, thus illustrating its constructive or destructive interference effect on the coupling values.

Another computational study on the effects of the polar environment on the TET coupling was focused on triplet exciton migration in polyA-polyT DNA sequences. ${ }^{178}$ By combining MD to account for conformational flexibility of the donor and acceptor sites and CIS-PCM calculations of $\mathrm{A}-\mathrm{A}$ and $\mathrm{T}-\mathrm{T} \pi$ stacks, it was shown that the polarization contribution to the coupling can considerably modulate its values in individual conformations. This effect can be traced to the perturbation of molecular orbitals of each fragment and thus to a change of their overlap upon solvation, which translates into a new electronic coupling value. However, when an average over many conformations is considered, an overall cancellation of the polarization effects is found. This findings when combined with the observation that $\mathrm{A}$ and $\mathrm{T}$ nucleobases (and their 
stacks) are very polar species seems to suggest that also in other TET systems, the triplet exciton mobility should not be very sensitive to the polarization effects of the environment.

This study, however, also confirms the important role of another (less direct) "environment" effect, namely the temperature-dependent conformational disorder. Due to the high sensitivity of the TET to distances through both exchange and overlap interactions in fact, it is of great importance to properly include the effects of structural fluctuations both within the single interacting units and their relative arrangement, as well as to account for a proper sampling of the solvent conformational space.

The fundamental role that structural fluctuations have on all energy transfer processes is exactly the subject of the next section.

\section{SPECTRAL DENSITIES, DISORDER AND CORRELATIONS}

The dynamic properties of the environment (or bath) play an important role in the quantum dynamics of open quantum systems. In electronic energy transfer processes, this role can easily be understood examining Förster's formulation of the rate based on the golden rule of quantum mechanics: ${ }^{179,180}$

$$
k_{E E T}=\frac{2 \pi}{\hbar} V^{2} J
$$

where $V$ is the electronic coupling among the donor and acceptor electronic states, and $J$ is the spectral overlap factor between normalized donor emission and acceptor absorption spectra. The $V^{2}$ term indicates that the chromophores must be electronically coupled for the transfer reaction to occur. The $J$ term, in turn, arises from the need to have resonant energy levels thus ensuring energy conservation in the process. Because typically the average energy levels of the donor and acceptor molecules are not in resonance, the resonance condition is fulfilled thanks to the dynamic fluctuations in energy levels due to electronic-vibrational coupling among electronic states and the nuclear degrees of freedom of the system. Such fluctuations are the origin of the broadening in the spectral line shapes of the molecules, so Förster theory determines the probability that the energy levels are in resonance from the spectral overlap of optical line shapes.

Because the spectral line shapes of the donor and the acceptor are typically extracted from experiments on the individual chromophores, Förster theory introduces two approximations in the determination of the spectral factor. A first approximation is the assumption of independent baths for the donor and the acceptor. However, the eventual presence of shared modes among the molecules could substantially impact the predicted rates. ${ }^{181}$ The other approximation is related to the treatment of static disorder. In 
principle, the spectral overlap should be obtained from the homogeneous donor and acceptor line shapes, and then be averaged over inhomogeneous broadening caused by static disorder, which arises from very slow fluctuations in the energy levels of the chromophores. ${ }^{148}$ In practice, though, the spectral overlap is obtained from line shapes individually averaged over static disorder.

In other theories tackling EET dynamics beyond the weak-coupling regime, like modified Redfield theory or the hierarchical equations of motion (HEOM) method, the information on how the electronic states couple dynamically with the many vibrations in the system also plays a key role. ${ }^{20,182}$ Indeed, increased attention to the dynamical characteristics of the exciton-phonon coupling has arisen from 2D electronic spectroscopy experiments performed in recent years on several photosynthetic pigmentprotein complexes and in conjugated polymers. ${ }^{183-200}$ These experiments have in fact unveiled unexpected long-lived quantum coherence effects in the energy migration process, an indication that EET occurs in an intermediate coupling regime. While the actual consequences of quantum coherence in the light-harvesting efficiency of these systems is still subject of a heated debate, understanding the molecular basis sustaining coherence effects have far reaching consequences, e.g. related to quantum computing technologies. ${ }^{201}$

So far, different hypotheses have been proposed to explain the molecular basis sustaining quantum coherence. First, it was proposed that the protein environment can protect coherence by sharing vibrational modes with several chromophores, thus leading to correlated fluctuations of the chromophore's energy levels. ${ }^{10,13,184,194,202-225}$. As we will describe later, this hypothesis has lost momentum after some theoretical works based on molecular simulations that suggest that such correlations are negligible in the light-harvesting complex 2 (LH2), the Fenna-Mathews-Olson complex (FMO) and the phycoerythrin 545 (PE545) antenna complex. ${ }^{124,226-229}$ Some correlations have been identified, however, arising from slow protein motions not captured by molecular simulations. $^{230}$ An alternative explanation gaining importance suggests that the structured-nature of the environment could mediate coherence effects through quantized vibrations coupled to the electronic states that commensurate the energy difference among exciton states. ${ }^{231-234}$

From this discussion it emerges that the dynamical interplay among electronic states and nuclear vibrations are key in order to understand the subtleties of energy transfer and light harvesting. In quantitative models of energy transfer and optical spectra of multichromophoric systems, typically the dynamical characteristics of the environment are condensed in the so-called spectral density function, $J_{i}(\omega)$, which describes the frequency-dependent system-bath coupling strength of the selected excitation of chromophore $i{ }^{235}$ An example of the spectral density of the $C h l b$ pigment of the watersoluble chlorophyll binding protein (WSCP) predicted from QM/MM excited-state calculations performed along classical and QM/MM MD trajectories, and compared to difference fluorescence line-narrowing (DFLN) data, is shown in Figure 2. ${ }^{139}$ 

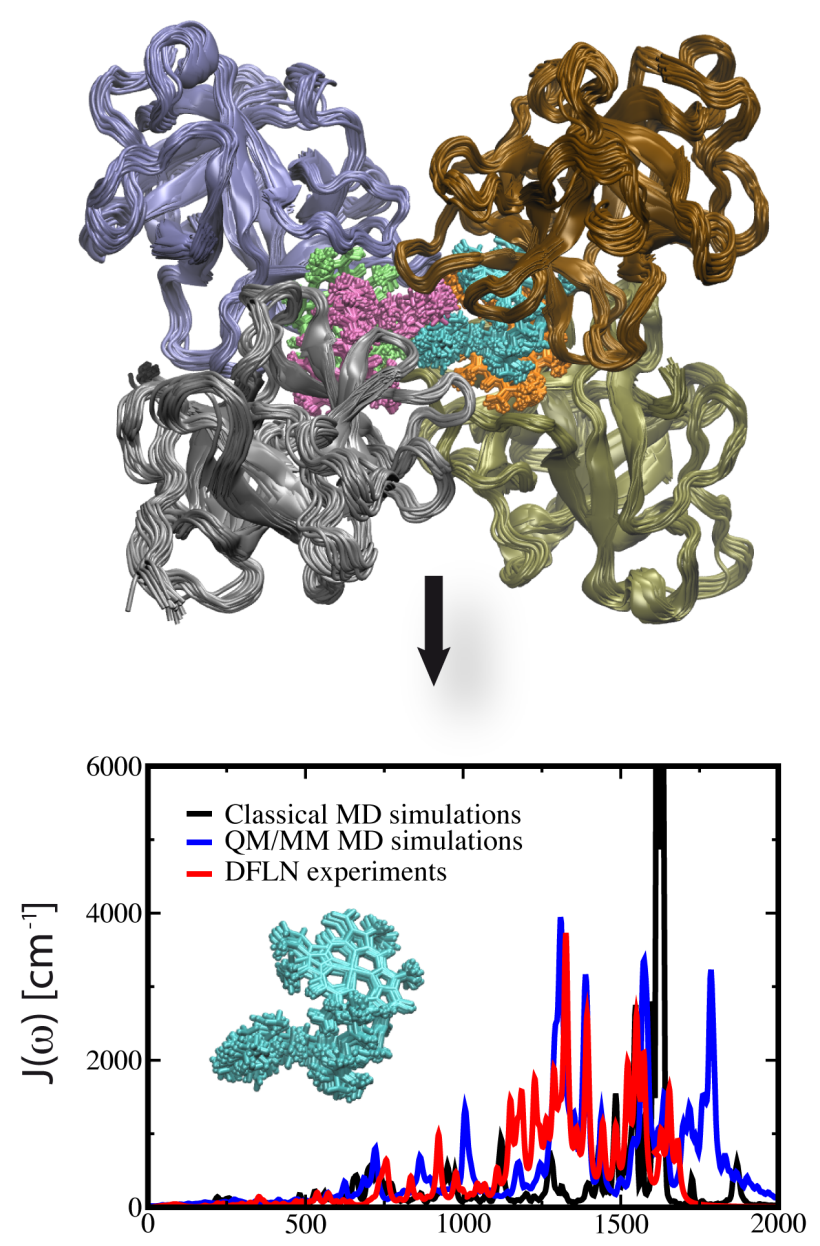

$\hbar \omega\left[\mathrm{cm}^{-1}\right]$

Figure 2. Spectral density of the $C h l b$ pigment of the water-soluble chlorophyll binding protein (WSCP) predicted from $\mathrm{QM} / \mathrm{MM}$ excited-state calculations performed along classical and $\mathrm{QM} / \mathrm{MM} \mathrm{MD}$ trajectories and compared to difference fluorescence line-narrowing (DFLN) data $^{139,236}$

For practical reasons, the spectral density is usually modeled as a sum of two terms:

$$
J_{i}(\omega)=J_{0, i}(\omega)+J_{v i b, i}(\omega)
$$

In eq $2.4, J_{0, i}(\omega)$ encapsulates the coupling to a continuum of low-frequency damping modes due to the environment, for example, the protein and the solvent surrounding a photosynthetic chromophore. In turn, the $J_{v i b, i}(\omega)$ term accounts for the coupling to high-frequency discrete modes. These latter arise mostly due to intramolecular vibrations of the chromophores, but also due to the environment, for instance, when the excited state is coupled to the surrounding environment through a hydrogen bond.

Different expressions are often used to describe $J_{0, i}(\omega)$, and recently more refined expressions have been proposed based on the modelling of delta fluorescence linenarrowing spectra of several photosynthetic complexes. In particular, Kell and coworkers $^{237}$ have shown that commonly used expressions like the 
Drude-Lorentz/constant damping Brownian oscillator describe a qualitatively wrong behavior when compared to experiment, specially at low frequencies, whereas a lognormal distribution exhibits the desired attributes for a physically meaningful phonon $J_{0, i}(\omega)$. On the other hand, the $J_{v i b, i}(\omega)$ term is typically described as a collection of harmonic oscillators.

Because of the importance that the structured-nature of the spectral density could have on the coherent characteristics of EET, much effort has been devoted in recent years in developing both experimental and theoretical strategies able to determine it. From an experimental point of view, the spectral density can be fit to different optical spectra, for example, in order to reproduce the fluorescence line shape or the dynamic fluorescence Stokes shift. ${ }^{238,239}$ This strategy however is not free from ambiguities, and more accurate determinations can be obtained from site selective spectroscopies, like spectral hole burning or delta fluorescence line-narrowing, ${ }^{23,237,240-244}$ which allow to directly estimate the exciton-phonon coupling constants, the Huang-Rhys factors, for each discrete vibration. An important limitation of the experimental techniques, however, is the inability to resolve the fine-tuning exerted by the local protein environment in the spectral densities of different chromophores in a given biological complex, as typically the data corresponds to an average of the low-energy chromophores that fluoresce. Indeed, experiments show that the shape of $J_{i}(\omega)$ varies among different pigment-protein complexes. ${ }^{236,242,243}$ Such fine-tuning could be important in order to understand if natural evolution has favored quantum coherence effects in photosynthesis, an indication that indeed coherence benefits the overall function of the photosynthetic machinery.

In order to overcome the limitations of experimental techniques, several research efforts have been recently directed toward the theoretical estimation of spectral densities based on two different strategies. The most common one relies on the exploration of the structural fluctuations of the system through classical MD simulations of the chromophore embedded in its environment (protein, solvent, etc), and the following evaluation of site energy autocorrelation functions by performing calculations of the site energy fluctuations along the classical trajectory using the methods described in section 2 . $^{124,227,245-263}$ The Fourier transform of the autocorrelation function then gives access to the spectral density of the chromophore including both exciton-vibrational interactions arising from intramolecular motions or from pigment-environment interactions. Because state-of-the-art MD simulations are presently limited to timescales of miliseconds, though, this strategy can not be used to estimate the very slow components in $J_{0, i}(\omega)$, thus precluding the possibility to relate low frequency modes in the spectral density to static disorder.

Alternatively, the spectral density can be obtained by first estimating the vibrational modes in the system through a normal mode analysis (NMA), and later evaluating the impact on the site energies along each normal mode. ${ }^{230,256,264}$ In this case, however, the contributions arising from intramolecular modes are calculated separately from those arising from the environment, so the coupling among the chromophore and the 
environment motions can not be included. ${ }^{256}$ An important advantage of this strategy, however, is the possibility to estimate static disorder by determining the low frequency modes in the spectral density, for example, through a NMA analysis of the protein motions in a pigment-protein complex. ${ }^{230}$

In the following, we discuss these two approaches as well as their main advantages and shortcomings. In addition, we also discuss the insights provided by these complementary techniques in order to understand the degree of correlation among site energies, and also eventual correlations involving electronic couplings, as these can significantly affect the quantitative modelling of light harvesting phenomena.

\subsection{MD-based approaches}

A natural strategy to estimate the spectral density of a chromophore embedded in a biological environment relies on the computer simulation of the site energy fluctuations arising from environmental vibrations. The size and complexity of biosystems, for example a photosynthetic pigment-protein complex solvated in water or located in a lipid membrane, precludes at present the possibility to perform such simulations using accurate quantum chemical methods for the description of the complete system. Thus, recent efforts in this direction have been based on classical MD simulations, in which the energy of the system is described by empirical functions called force fields, calibrated and parameterized by comparison to a plethora of experimental and theoretical data. MD techniques are a well-established technique, routinely used in studies of drug design, enzymatic catalysis or molecular recognition. Once the structural fluctuations of the system are obtained, usually corresponding to the ground state, the corresponding trajectories are used to perform a posteriori calculations of the site energy fluctuations. These are typically based on the methodologies described in section 2, based either on mixed QM/MM calculations or more simple and efficient electrostatic shift expressions, given the need to account for the impact of the environment on the energy shifts. As it will be discussed later, the mismatch among the QM/MM level of theory used for the excited state calculations and the classical geometries determined by the classical force field is an important problem that can introduce significant errors.

As said, the spectral density can be computed from the Fourier transform of the site energy autocorrelation function as: $:^{247,252,254}$

$$
J_{i}(\omega)=\frac{\beta \omega}{\pi} \int_{0}^{\infty} C_{i}^{c l}(t) \cos (\omega t) d t
$$

where $\beta=\frac{1}{K_{B} T}$ and $C_{i}^{c l}(t)$ is the classical autocorrelation function of the fluctuation of the site energies. As it was recently clarified by Valleau and co-workers, ${ }^{254}$ eq 3.4 includes a classical prefactor to nullify the temperature dependence of the correlation function, given that $J_{i}(\omega)$ is obtained from a classical autocorrelation function. 
By evaluating the site energy fluctuations along the MD trajectory, the autocorrelation function can be obtained using the following discrete expression:

$$
C_{i}^{c l}\left(t_{j}\right)=\frac{1}{N-j} \sum_{k=1}^{N-j} \Delta E_{i}\left(t_{j}+t_{k}\right) \Delta E_{i}\left(t_{k}\right)
$$

where the superscript in $C_{i}^{c l}\left(t_{j}\right)$ indicates that it is a classical autocorrelation function of chromophore $i$, and $N$ is the number of temporal data containing the site energy fluctuations.

The practical application of these expressions to the computation of the spectral density deserves some discussion. Because in principle one should be interested in sampling all relevant timescales of the system, ranging from high-frequency intramolecular modes of the chromophores to slow motions of the protein that cause static disorder, the autocorrelation function should be determined along sufficiently long simulation times. Moreover, such autocorrelation functions derived from simulations have to be computed many times in order to be able to obtain the correct statistical average. In order to reduce the computational cost associated to the huge amount of QM calculations of site energies, such calculations are typically performed over discrete time steps of 2-5 femtoseconds, in order to capture the fastest oscillations in the system coupled to the excitation energies due to bond stretchings among heavy atoms. ${ }^{124,227,247,248,252-263}$ Still, the considerable cost of QM calculations precludes the sampling of long time scales in the evaluation of eq 4.4, typically performed over a few picoseconds, where the main features of the autocorrelation function decay. Thus, the slowest motions of a protein complex characterized by frequencies below $\sim 50-100 \mathrm{~cm}^{-1}$, can still not be captured by this approach, as these modes oscillate in the picosecond or longer timescales. An interesting alternative, in this case, was recently proposed by Renger and co-workers based on a normal mode analysis of a protein complex, ${ }^{230}$ as it will be described in next section.

One of the main advantages of MD-based approaches, in any case, is the possibility to simultaneously determine the continuous (phonon), $J_{0, i}(\omega)$, and the vibrational, $J_{v i b, i}(\omega)$, contributions to the spectral density. Thus, the modulation of intramolecular chromophore vibrations by the surrounding environment can be fully taken into account, although described through approximate classical force fields. This coupling has been shown to introduce important differences, for example, in the low and medium frequency features of the spectral density of dihydrobiliverdin (DBV) and phycoerythrobilins (PEB) pigments in the PE545 main light-harvesting antenna complex of cryptophyte algae both in terms of vibrational frequencies and coupling strengths. ${ }^{259,261}$ Thus, whereas such low-frequency features have been shown to arise from slow intramolecular motions of the bilin pigments (in contrast, for example, to the chlorophylls of the FMO complex, where they arise from intermolecular interactions with the protein environment $)^{259}$ the local environment that embeds the pigments still considerably modulates the intramolecular vibrations of the bilins in PE545, ${ }^{261}$ probably 
owing to the flexible nature of bilin pigments characterized by a linear tetrapyrrole structure.

One way to investigate how the protein scaffold modulates the pigments' vibrations is by comparison of modes obtained either by a standard normal mode analysis (NMA) or from a quasi-harmonic analysis $(\mathrm{QH})$ of the MD trajectory. The NMA analysis probes the vibrations around a single minimum, while the $\mathrm{QH}$ analysis aims at characterizing the global extent of the configurational space accessible to the system during an MD simulation. ${ }^{265}$ This is achieved by diagonalizing the mass-weighted covariance matrix of atomic positions in Cartesian coordinates, and is typically used to determine configurational entropies of biomolecules. The corresponding eigenvectors describe motional modes (quasi-harmonic modes) around the average system configuration. In order to characterize the features in the spectral density of individual pigments, the $\mathrm{QH}$ analysis can be applied to the MD trajectory containing only the coordinates of the selected pigment. The comparison between the NMA and QH modes allows assessing the impact of the protein and the solvent environment in the intrinsic vibrations of the pigments. In the pigments of the PE545 complex, such comparison showed that the accumulation of modes around 1000 and $1500 \mathrm{~cm}^{-1}$ observed for the single pigments was considerably smoothed out when their vibrations are coupled to the protein motions. ${ }^{261}$ In addition, comparison of predictions obtained for the isolated or solvated complex showed that the solvent can also considerably redistribute the vibrational frequencies in the spectral density.

The modulation of low-frequency features by the environment - together with the tuning of the site energies of the pigments - could potentially be involved in the optimization of quantum coherence effects in photosynthetic complexes, as suggested by recent theoretical work. ${ }^{223,231-234}$ However, the considerable uncertainty regarding the accurate calculation of both spectral densities and site energies precludes at present a robust assessment of this hypothesis.

The application of the MD-based technique to the determination of the spectral density of several photosynthetic pigment-protein complexes, in fact, has shown that the results depend quite significantly on the QM model chemistry used and the force field selected to describe the system in the MD simulation. This is particularly evident in predictions of the spectral density of the FMO complex, recently studied by several research groups using a variety of approaches, including TD-DFT, ZINDO and different classical force fields like CHARMM or AMBER. ${ }^{227,229,252-254,257-259,262}$

In this case, two careful analyses performed independently by Chandrasekaran et al. ${ }^{262}$ and by Wang et al. ${ }^{229}$ seem to indicate that the choice of force field is the most delicate one in predictions of the FMO spectral density. Further insights in this issue have emerged from a study by Kim and co-workers, ${ }^{263}$ who investigated the effect of the mismatch among the classical potential energy surface (PES) used in MD simulations and the QM level of theory adopted for a solvated coumarin-153 chromophore by 
deriving accurate force fields able to describe the QM PES. Interestingly, their study indicates that the adoption of approximate force constants in the force field terms have a significant impact on the spectral density, although the approximate equilibrium constants have also a significant effect. Thus, further improvement on the quantitative accuracy of spectral densities derived from these approaches could be based on the development of specialized force field able to accurately describe the QM PES of the chromophore, or in QM/MM MD simulations where the chromophore is described at a QM level. Indeed, this latter strategy has been shown to provide an drastically improved description of the spectral density for the $C h l a$ and $C h l b$ chromophores of the watersoluble chlorophyll binding protein, although low-frequency features seem to degrade somewhat at the QM/MM level due to inaccuracies in the treatment of the interactions among the QM and MM regions, as illustrated in Figure 2. ${ }^{139}$ All these approaches, however, still rely on classical MD simulations, even if the PES is described at the QM/MM level, and a further source of errors in the prediction could arise from the neglect of quantum effects in the dynamics.

\subsection{NMA-based approaches}

A strategy based on normal mode analysis has been recently proposed for the evaluation of spectral densities by several authors, with the aim of overcoming the main limitations of MD-based approaches. Namely, the mismatch among the levels of theory used in the MD simulation and the excitation energy calculations, ${ }^{256,264}$ or the inability to sample very slow frequency motions of biomacromolecules responsible for static disorder. ${ }^{230}$ In this strategy, however, the calculation of the phonon, $J_{0, i}(\omega)$, and the vibrational, $J_{v i b, i}(\omega)$, contributions to the spectral density are uncoupled and computed separately, an effect which has been shown to impact the low-frequency region of the spectral density. ${ }^{261}$

When applied to the calculation of the intramolecular contributions to the spectral density, this strategy allows to perform the NMA analysis and the assessment of the impact of the chromophore vibrations on the excitation energies at accurate QM levels of theory, thus minimizing or eliminating the geometry mismatch problem discussed in the previous section for classical MD-based strategies. Thus, TDDFT methods have been recently applied to the estimation of the electronic-vibrational coupling of the $B C h l a$ pigments in the reaction center of purple bacteria ${ }^{256}$ and a perylene bisimide chromophore. ${ }^{264}$ This strategy has been shown to provide more realistic coupling strengths for the high-frequency modes of $B C h l a$, typically severely overestimated in MD-based approaches, and provide overall vibrational frequencies and Huang-Rhys factors in reasonable agreement with experiments.

The NMA approach has also been applied by Renger and co-workers to the prediction of the electron-phonon coupling, i.e. the contributions arising from the continuum of 
slow modes due to the environment surrounding the chromophores. ${ }^{230}$ In this case, a minimization and NMA analysis of the FMO pigment-protein complex was performed using a classical force field, while keeping the intramolecular pigment modes frozen, and then the modulation of the electronic transition energies along each mode coordinates were estimated using the electrostatic shift method (see section 2.2.2). The important advantage of this strategy is the possibility to assess the impact of very slow protein motions on the spectral density, not accessible from MD-based approaches due to the limited MD times that can be simulated. Encouragingly, this study has shown that the strategy is capable of estimating the spectral density of electron-phonon coupling with a reasonable agreement with experiments. A potential drawback of the method, however, is the assumption of a single minimum in the NMA analysis. Indeed, protein energy landscapes are typically characterized by multiple minima and this could impact the predicted spectral densities.

\subsection{Correlations}

There has been much speculation on the potential protection of quantum coherence effects in photosynthetic light harvesting by the protein environment, through a mediation of the correlated fluctuations on the site energies of the pigments if they are coupled to shared protein vibrations. ${ }^{10,13,184,194,202-225}$ Renger and co-workers introduced a simple expression describing an exponential decay for such correlations with the interpigment distance, $\exp \left\{-R_{m n} / R_{c}\right\}$, where $R_{m n}$ is the separation between molecules $m$ and $n$, and $R_{c}$ is a phenomenological correlation radius. A value $R_{c}=5 \AA$ was estimated from simulations of time-resolved pump-probe data of photosystem II reaction centers ${ }^{266}$ and found to explain also decay constants for the heterodimer of the water-soluble chlorophyll binding protein (WSCP). ${ }^{267}$

Recent investigations based on the MD-based strategies described in section 4.1, however, have not found any significant correlations among the site energy fluctuations of the pigments in the photosynthetic complexes LH2, ${ }^{124} \mathrm{FMO}^{226,227,256}$ and PE545. ${ }^{228}$ Recently, Wang and co-workers found significant correlations in the FMO complex due to pigment-protein interactions estimated using electrostatic shift calculations. ${ }^{229}$ Those, however, were then overwhelmed by the fluctuations due to the chlorophylls internal vibrations described by ZINDO calculations. In DNA, in contrast, significant correlations of 0.7 and 0.4 between direct neighbors and second neighbors have been found, ${ }^{268}$ correlations that should strongly affect exciton dynamics. ${ }^{269}$ In addition, significant mixed correlations involving site energies and electronic couplings have been predicted for the PE545 complex, ${ }^{228}$ which have been suggested also to impact quantum coherence and energy transfer dynamics. ${ }^{217,219}$ For the FMO complex, also cross-correlations between energies and couplings have been predicted when the latter are computed using the PDA. However, when the coupling is obtained taking into account the shape of the transition densities using the TrEsp method, the correlations turned out to be negligible. ${ }^{226}$ The more flexible structure of the bilin pigments in 
PE545, characterized by a linear tetrapyrrole arrangement, could explain this difference compared to chlorophylls, as rotation of a torsion among pyrrole rings could simultaneously modulate the site energy and the transition dipole orientation, thus also changing the couplings with other chromophores.

As suggested by Jing and co-workers, ${ }^{256}$ site energy correlations in the FMO complex could be hidden in the inhomogeneous broadening due to the limited MD simulation times. Indeed, it is quite intuitive that slow protein motions in timescales larger than those sampled in these studies, typically a few hundred of picoseconds, could couple simultaneously to several pigments. This possibility was explored by Renger and coworkers using the NMA-based strategy described in the previous section, which allows assessing the impact of very slow protein motions on energy correlations. ${ }^{230}$ Interestingly, that study indeed showed that strong correlations are found for very low frequency modes. However, their impact on exciton relaxation was found to be minor, suggesting they don't have any relevant biological role. In any case, these correlations have been shown to be important in order to interpret two-dimensional electronic spectra. ${ }^{270-272}$

\section{APPLICATIONS}

\subsection{Artificial light harvesting systems}

The energy transfer phenomena of natural LH systems gave great inspiration for the design in artificial photofunctional devices, including photovoltaic, electroluminescent, and biomedical devices. ${ }^{273-282}$

Promising candidates for artificial light harvesting should transfer excitation energy with very high efficiency and have a clear antenna effect, namely the ratio of the emission intensity of the acceptor upon excitation of the donor to that of the direct excitation of the acceptor should be significantly larger than 1. To achieve these parameters, the chromophores need to have proper spatial organization (favorable spacing and orientation) and distinct spectroscopic properties. The former is key to minimizing energy loss (for example, by avoiding self-quenching), and the latter is required to form so-called energy gradients that facilitate the energy flow from antennas to energy sinks.

Obviously, the first example of artificial device one can think of is that exploiting the intramolecular EET in molecular dyads of covalently linked donor-acceptor moieties. However, with the evolution of energy-transfer systems toward increasingly large and more sophisticated assemblies, the reliance on covalent linking has become not feasible. Noncovalent interactions, including van der Waals, hydrophobic, $\pi-\pi$, dipole, and metal ligation, become thus a powerful alternative as giving an infinite number of possibilities 
to construct self-assembled architectures for various applications without timeconsuming synthesis.

Self-assembly of a chromophore-derived macromolecule is indeed an extremely valid strategy for artificial light harvesting. ${ }^{283}$ Dendrimers in particular have been widely used due to their well-defined tree-like structures, ${ }^{284}$ providing predetermined sites for grafting chromophores. ${ }^{285}$ Moreover, recent studies demonstrated the utility of biological materials, ${ }^{279}$ including proteins and DNA, as templates to guide nanoscale organization of covalently or electrostatically bound pigments and obtain hybrid biomaterials with interesting potentials in achieving highly efficient light harvesting.

Despite their very interesting properties the cost of these hybrid biomaterials may hamper their scale up and widespread applications in artificial light-harvesting systems. Recently, organic-inorganic hybrid materials attracted considerable attention for their low costs and excellent mechanical properties, which are required for device applications. Within this area, large interest has been focused on quantum dots ${ }^{286}$ and plasmonic nanostructures. ${ }^{287}$

In the following, a selection of studies on different classes of artificial supramolecular light-harvesting systems is reviewed. The necessarily limited number of reported examples has been focused on those which are based on the quantum-chemical strategies we have described in the previous sections.

\subsubsection{Bridged dyads and dendrimers}

Intramolecular EET in molecular dyads ${ }^{288}$ has been studied in great detail over several decades in order to learn about the underlying mechanisms, especially in terms of how the rate varies with distance and orientation. In this context, structures where the EET units are linked by rigid linkers (also called "molecular wires") are to be preferred to flexibly-linked molecular dyads. Systems containing perylene imide chromophores, metal and metal-free porphyrins as well as boron dipyrromethene (Bodipy) dyes have been largely used for their specific characteristics as both light absorbers and EET donors/acceptors. In particular porphyrins are ideal chromophores for artificial light harvesting because they are structurally similar to photosynthetic pigments and have advantageous photophysical properties, such as photostability, visible-light absorption, long-lived excited states, rapid excitation energy exchange, and high molar extinction coefficients, which can be easily tuned by metal coordination.

As described in section 3.1.3, the presence of a molecular linker (bridge) between the $\mathrm{D} / \mathrm{A}$ pair in the dyad can induce specific through-bond (TB) effects involving either superexchange or polarization effects or a combination of the two. In particular, the presence of $\pi$-conjugated bridges consisting of covalently connected identical repetitive molecular motifs has been largely studied for their characteristics which make them 
promising candidates for mediating charge/energy transfer over long distances, namely their rigidity and rod-like structure and more importantly their high degree of electron delocalization.

Recently, an analysis based on the QM/MMPol approach described in section 3.1.2.1 has been used to dissect the different contributions to the TB effect in dyads of the type D-bridge-A. Three different models were introduced (see scheme 2): (a) model M0, a full-QM approach which neglects the bridge; (b) model Mc, a full-QM approach, which includes the bridge at QM level by adding it (fully or partially) to the D or A fragments; and (c) model MMPol, a hybrid QM/MM approach where the bridge is described using a polarizable MM description.

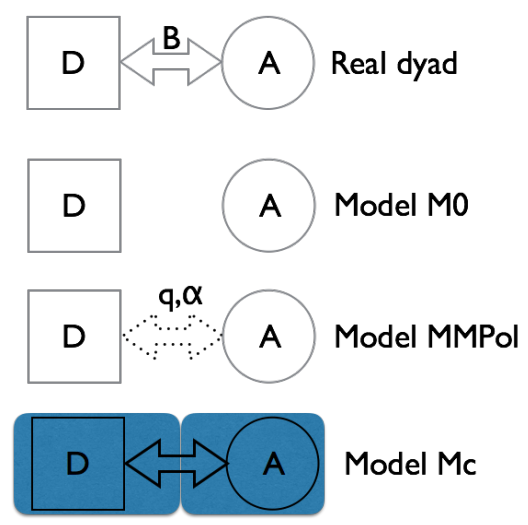

Scheme 2

In all models, hydrogens were used to saturate the free valences of the various fragments whereas the effect of the solvent was introduced through a PCM description (see section 3.1.2.1). This analysis has been applied to different dyads: a perylene diimide chromophore (PDI) linked via a triphenylene bridge to a terrylene diimide (TDI) chromophores, ${ }^{148}$ a perylene monoimide (PMI) bridged to a Ph-TDI through a ladder-type pentaphenylene $(\mathrm{pPh})$ spacer $^{151}$ and various dimers formed by zinc- and free-base- porphyrins with a diaryl-ethyne linker. ${ }^{152,289}$ In all cases a TDDFT description was used for the calculation of the electronic couplings through a direct use of the full transition densities (see eqs 1.3, 16.3 and 20.3). The comparison of the three models allows a double analysis: (i) by comparing M0 and MMPol, the polarization effects of the bridge which can enhance or screen the coupling can be quantified and (ii) by comparing Mc and MMPol the superexchange effect can be estimated. It was found that not only the TB contribution largely varies with the nature of the D-bridge-A system but also that the same type of bridge can act in very different ways when changing the linking D and A moieties.

For example in the case of porphyrin-based systems, ${ }^{289}$ a purely screening effect leading to a reduction of the EET rate was found for a given choice of the bridge and the porphyrin pair while this effect represented only a small contribution of the full TB 
effect when the same bridge was linked to two differently substituted porphyrins. In the latter case, the main TB effect was better interpreted as a QM superexchange. To quantify it, the so-called "mediation rate constant" introduced in previous analyses ${ }^{290}$ and defined as the difference between the full rate and the through-space (TS) contribution, was calculated as $V_{m e d}^{2}=V_{M c}^{2}-V_{M M P o l}^{2}$. By using bridges of different lengths, $V_{\text {med }}^{2}$ was fitted versus the $\mathrm{D} / \mathrm{A}$ distance $(\mathrm{R})$ with an exponential function $A \mathrm{e}^{-\beta \mathrm{R}}$ and a very good fitting was found when the parameter $\beta$ (the so-called "attenuation factor") was made solvent-dependent. Indeed, several studies have shown that the use of the $\beta$-value as a simple descriptor of "conduction" is rather limited as this is not a bridge specific parameter, but instead a donor-bridge-acceptor ensemble parameter. Effects of the fluctuations on the relative orientation of the D-bridge and bridge-A were also investigated through a scan of the corresponding dihedral angles. It was found that classical contribution to the coupling is favored by the configuration, where the two porphyrins are coplanar and perpendicular to the bridge plane, which probably corresponds to the smallest screening induced by the bridge. Conversely, the superexchange contribution, favored by the communication between the $\pi$ orbitals of the porphyrin and those of the bridge, tends to its maximum when the whole system lies on the same plane. Finally, the variation of the zinc-porphyrin angle with the bridge affects the coupling more than the corresponding variation of the free-porphyrine.

A related study on the TS and TB contributions to the EET rate was recently presented on BODIPY-anthracene dyads separated by conjugated bridges of different lengths. ${ }^{291}$ The through-space contribution to the EET rates was calculated either in the PDA or from monomer transition densities using a DFT/MRCI description. Furthermore, the influence of the nuclear motion was taken into account based on short ab initio Born-Oppenheimer MD trajectories. For all dyads in which the transition dipole moments of donor and acceptor are oriented in a parallel manner, EET rates were found in the order of the temporal resolution of the experiment when a fragmentation model in which the bridge is included in the donor moiety (e.g. something similar to Mc model) is used. If the simpler fragmentation model completely neglecting the bridge (e.g. M0) is applied, the EET rates become significantly too small. For dyads, in which the transition dipole moments are oriented in a perpendicular manner, dynamic effects play an important role, increasing the computational EET rate by up to 10 orders of magnitude, compared to the static model.

The dynamic properties of the bridge giving rise to different conformations are indeed known to be extremely important in determining the EET rate especially when involving triplets. Among the first to show experimentally the influence of the bridge conformation on the donor-acceptor electronic coupling in D-B-A systems were Harriman and co-workers. ${ }^{292}$ They probed the effect of torsional angles within the bridge structure on the rate of intramolecular triplet excitation energy transfer $\mathrm{k}_{\text {TET }}$ in a series of ruthenium(II)/osmium(II) (tpy)2 donor-acceptor complexes linked by 
ethynylene-substituted biphenyl bridges. Another approach to investigate how bridge conformation affects the flow of excitation energy or electrons between the donor and acceptor in D-B-A systems is to use temperature as a way to tune the Boltzmann distribution of conformations. Albinsson and co-workers have investigated the temperature dependence of TET, both experimentally and theoretically, in a series of zinc(II), and free-base porphyrin separated by 2-5 oligo-p-phenyleneethynylene units. $^{293}$
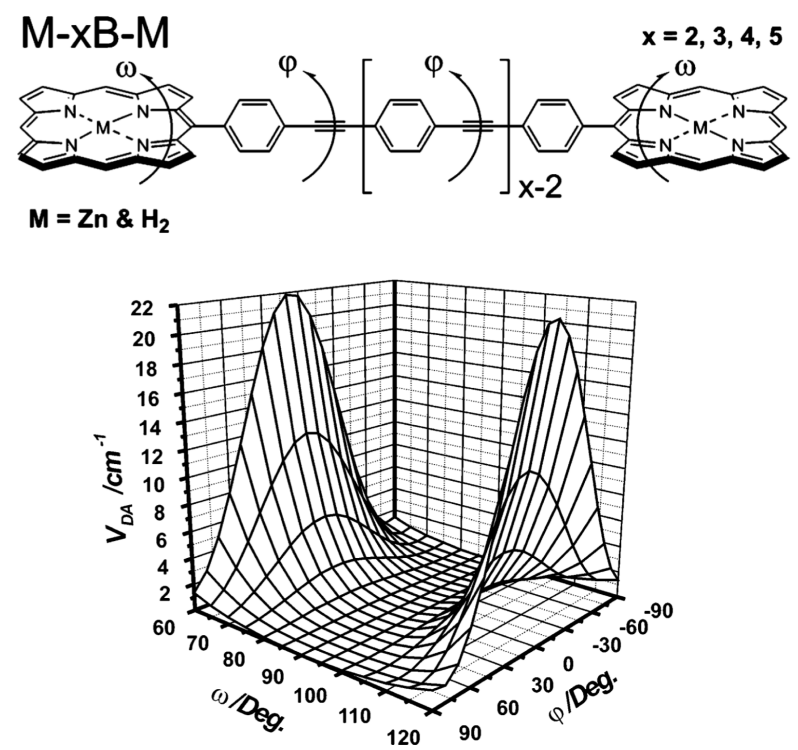

Figure 3. The porphyrin-bridge $(\omega)$ and internal bridge $(\varphi)$ dihedral angles used to model the conformational dynamics and the calculated electronic coupling, $V_{D A}$, for combinations of various dihedral angles. Reprinted and modified with permission from Ref [293]. Copyright 2006 American Chemical Society.

In that study, the transfer rates showed exponential distance dependence for the entire temperature range, but more interestingly the attenuation factor, $\beta$, was found to be temperature dependent. In parallel to these experiments, a theoretical model was derived to calculate the total electronic coupling, based on a Boltzmann distribution of the bridge conformations. The procedure involved DFT calculations of the potential energy surfaces as a function of the porphyrin-bridge angle rotation $(\omega)$ and the bridge unit rotations $(\varphi)$ (see Figure 3 ) followed by calculation of the total electronic coupling $V_{D A}$ for each conformational distribution derived from half the triplet excitation energy difference (calculated at TDDFT level) between the two lowest triplet excited states at the avoided crossing geometry. The average electronic coupling for the $\mathrm{M}-\mathrm{xB}-\mathrm{M}$ systems was then rewritten as $\left\langle V_{D A}\left(\omega, \varphi_{1}, \varphi_{2} \ldots \varphi_{x-1}\right)\right\rangle=V_{x}\langle V(\omega)\rangle\langle V(\varphi)\rangle^{x-1}$

From this equation it can be seen that the factor containing the porphyrin-bridge angle, $\mathrm{V}(\omega)$, will only shift the coupling by the same amount for all compounds in the series and will, thus, not affect the distance dependence. The distance dependence will only be 
governed by the constant factor, $\mathrm{V}_{\mathrm{x}}$, and the factor describing the bridge angle dependence of the electronic coupling, $V(\varphi)$. Comparison of the theoretical and experimental results suggests that the bridge conformation, described by the dihedral angles between the individual phenyl groups of the bridge, can be considered to be totally randomized on the time-scale of the transfer process.

Besides their important role in furthering fundamental knowledge, small (dyads and triads) systems are not especially useful light-harvesting units since they rarely collect more than $30 \%$ or so of the visible spectral range. There are additional problems relating to self-absorption and chromophore density and, in almost all cases, molecular dyads fail to fulfill the essential requirements inherent to a viable sensitiser for solar cells. Larger arrays are needed that overcome these issues: a possible strategy is that of using the layered structures inherent to most classes of dendrimers. ${ }^{294}$

Key to their success is a rapid transport of electronic excitation energy from peripheral antennas to photochemical reaction cores. Such energy transfer is conventionally attributed to either dipole-dipole interactions (Förster mechanism) or the overlapping of donor and acceptor electronic wave functions (Dexter mechanism). In dendrimeric structures, however, a fundamental role is played by the geometry which can take the branches from the weak coupling to the strong coupling regime.

This behavior was seen in a series of small phenylacetylene dendrons. ${ }^{295}$ By using ab initio CASSCF and CASPT2 calculations, the presence of large electronic coupling in the emitting geometry that is not seen for the absorbing geometry of the same molecules was evidenced and understood in terms of a variable excitonic coupling between the phenylacetylene branches. The origin of this geometry-dependent coupling was investigated through the interpretation of the calculations in terms of Förster, Dexter, and through-bond charge-transfer interactions. To dissect these effects a modified Harcourt model (see section 3.1.3) was used. It was found that the first Coulomb-like term of the Harcourt eq 23.3 remains nearly constant as the geometry relaxes, while the charge-transfer term increases from the absorbing to emitting geometries, despite the absence of large changes in charge distribution. A comparison of meta- versus parasubstituted phenylacetylenes was also presented showing that the assumption that metaconjugation effectively prevents electronic communication between dendrimer arms is only true at the ground state geometries. The fact that the absorption spectrum undergoes little change as more segments are added is due to a fortuitous cancellation of coupling terms and not, as previously assumed, due to an intrinsically small coupling between the arms. When this balance is perturbed by nuclear relaxation on the excitedstate potential energy surface, strong excitonic couplings are found.

The role of nuclear relaxation and dynamics in energy transfer processes of dendrimers has been further analyzed through nonadiabatic dynamics simulations. In particular the nonadiabatic excited-state molecular dynamics (NA-ESMD) framework developed by 
Tretiak and coworkers ${ }^{296}$ has shown to achieve an efficient and accurate description of ultrafast unidirectional energy transfer in dendrimers and in other extended conjugated molecular systems. The method combines the CIS formalism with a semiempirical Hamiltonian and treats quantum transitions among multiple adiabatic excited state potential energy surfaces through the fewest-switches surface hopping (FSSH) algorithm. ${ }^{297}$ Excited-state calculations are performed using the collective electronic oscillator (CEO) approach. ${ }^{298}$ The main finding of these studies is that in dendrimers with a dense manifold of excited states (such as in $\pi$-conjugated systems), strong exciton-phonon coupling and thermal disorder are the principal contributors to the ultrafast dynamics. More specifically, the electronic energy-transfer mechanism involves the ultrafast collapse of the photoexcited wave function due to nonadiabatic electronic transitions. The localization of the wave function is driven by the efficient coupling to high-frequency vibrational modes leading to ultrafast excited-state dynamics and unidirectional efficient energy funneling. ${ }^{299}$

\subsubsection{Conjugated polymers and organic crystals}

Energy gradients, or cascades, play a central role in energy transport. In most organic materials, energy is readily exchanged between electronic and vibrational degrees of freedom, with the consequence that excitons very effectively follow downhill energy gradients. This mechanism through which excess energy is converted into vibrations suppresses back transfer, while permitting further down-hill transport.

Cascade effects have been observed in conjugated polymers (CP). Upon photoexcitation, the initially created exciton can be delocalized both along and between polymer chains. The energy landscape for these excitons is governed by intra- and interchain conformations as well as dynamics.

In the idealized sense, the conjugated network of a CP may be formally extended over the entire polymer chain. However, in reality torsions and energetic disorder along the chain as well as chemical defects limit the persistence length of a given $\pi$-conjugated domain. We can imagine to cut a large conjugated polymer chain into a series of linked "chromophoric" units in which the electronic coupling between domains can be both through-space via Coulomb coupling and through-bond via $2 p$ orbitals of the carbon atoms of nearest neighbor segments. This implies that the diffusion and mobility of an excitonic state through a conjugated polymer material will be determined by strong and weak electronic couplings. Therefore, a Förster hopping model may not adequately describe energy transfer in these materials. A possible approach to calculate the coupling in polymers is to adopt the so-called "line-dipole", approximation. ${ }^{300}$

The idea is exactly that of partitioning the transition density of the whole system into corresponding subunits and to define a fractional transition dipole moment for each of these subunits. A numerically convenient way to decompose the total transition dipole 
moment into fractional dipoles is according to the sum rule

$$
\mu_{i A}=\sum_{a} \mu_{i A}^{(a)}, \quad \mu_{i A}^{(a)}=\mu_{i A} \psi_{i A}(a)
$$

where $\mu_{i A}^{(a)}$ is the transition dipole of the subunit $a$ and $\psi_{i A}(a)$ represents a discrete wave function defined on the basis of the subunits. The Coulomb integral for a dimer of two straight polymers yields approximately as

$$
V_{i A j B} \approx \sum_{a=1}^{L_{A}} \sum_{b=1}^{L_{B}} \psi_{i A}(a)\left(\frac{\mu_{i A} \mu_{j B}}{\left|\mathbf{r}_{a b}\right|^{3}}-3 \frac{\left(\mu_{i A} \mathbf{r}_{a b}\right)\left(\mu_{j B} \mathbf{r}_{a b}\right)}{\left|\mathbf{r}_{a b}\right|^{5}}\right) \psi_{j B}(b)
$$

This approximation of the excitonic interaction is called the "line-dipole" approximation, as the fractional transition dipoles follow the "line" of the polymer chain. In order to use the "line-dipole" approximation for bent polymers one has to consider the changing orientation of the subunit dipoles by a transformation which maps the straight polymer chain to the curved real polymer chain. For strongly bent or even kinked polymers, the wave function is partitioned at the kinks, and each segment between two kinks is assumed to be an independent spectroscopic unit.

Alternatively to the "line dipole" approximation also a monopole approximation (similar to that used in the TrEsp approach described in section 3.1.1) has been used where the atomic charges are here fitted so to reproduce the total transition dipole corresponding to the selected excitation. ${ }^{301}$ This approximation has been used to investigate the relative efficiencies of interchain versus intrachain energy-transfer processes in conjugated polymers by using semiempirical Hamiltonians in combination with a CIS description of the excited states. ${ }^{16,302,303}$ The calculations predict faster intermolecular energy transfer as a result of larger electronic matrix elements and suggest a two-step mechanism for intrachain energy transfer with exciton hopping along the polymer backbone as the limiting step. Successive studies based on the same monopole approximation for the electronic coupling have been extended to TDDFT calculations combined with trajectories from classical molecular dynamics simulations and applied to study the exciton migration dynamics in amorphous polymeric semiconductors. ${ }^{304}$ To reproduce the electrostatic environment of each polymer chain, the polarization of the QM subsystem by the surrounding charges residing on the MM atoms of the neighbor chains were incorporated (the values of the point charges were the same used for the force field). The main finding was that the interchain excitonic couplings for amorphous MEH-PPV are usually small suggesting that the transport mechanism across chain can be described by incoherent hopping.

Another aspect that has been investigated by combining quantum chemical methods and the monopole approximation of the transition densities is the validity of the nearest- 
neighbor approximation (NNA). This approximation considers only the interaction between the two adjacent molecules in a one-dimensional (1D) stack (four in a twodimensional (2D) layer) and thus reduces the task to a single "dimer" problem. The NNA is still widely used due to the extensive reduction of the computational effort. Calculating the exciton couplings in idealized 1D assemblies of stacked oligomers with different repetition units, it has been demonstrated that long-range interactions with remote non-nearest neighbor molecules become of importance for long oligomers and polymers, and cannot be neglected in the calculation of exciton couplings in molecular assemblies. ${ }^{305}$ In the same study, the impact of the self-screening of the molecules in the crystal has been also investigated by weighting the excitonic couplings by a distancedependent $s$ factor as that proposed within the PCM scheme (see eq 18.3). The results showed the expected decrease in the stack exciton coupling after correction for screening compared to the bare interactions, even if the coupling peaks at approximately the same length with or without the correction. This was explained by the fact that the shift in the permittivity going from the dimer to the full 1D array is primarily due to the next nearest neighbor interactions (at intermediate distances) that are only weakly affected by the screening function.

Among the materials for exciton transport, organic crystals are among those which have been more largely investigated from the theoretical point of view. ${ }^{306-308}$ Their favorable electronic properties arise from delocalized and highly polarizable $\pi$-electrons that support mobile charge carriers. The theory of exciton transport in organic semiconductors relies on two contributions. The first is the magnitude of the electronic coupling, which depends on the relative arrangement of the molecules in the crystal, whereas the second is the geometric relaxation of the molecule and its surroundings (reorganization energy).

While it has been traditionally assumed that low-energy excitations in organic semiconductors are of single-molecule character (Frenkel type) due to weak solid-state screening and intermolecular interactions, there is experimental and computational evidence for charge-transfer (CT) excitons in organic solids such as in oligoacene crystals. In this context, a diabatization scheme capable of computing the total excitonic coupling (short- and long- range) between multiple excited states (e.g., between FE and CT states) should be preferred. A general diabatization scheme to compute the excitonic couplings between multiple excited states in molecular dimers has been proposed by Arago and Troisi ${ }^{309}$ using an algebraic procedure to find the diabatic states. By applying this scheme at the TDDFT level by using a long-range corrected density functional to the tetracene crystal dimer, the authors have shown that the excitonic couplings computed within the three-state approximation present a continuous behavior and do not suffer from a breakdown as it is the case when only two states are considered. Additionally, by combining the scheme with the PCM model in its standard linear response implementation (nonequilibrium) (see section 2.2.1), they have shown that the 
coupling between one Frenkel state and a charge-transfer state is increased when augmenting the relative dielectric constant of the solvent. This suggests that, in the crystal environment, a larger mixture between FE and CT states than in vacuum should be expected.

A similar diabatic approach has been used $^{310}$ to show that the excitonic coupling in molecular crystals of anthracene and tetracene undergoes a very large fluctuation at room temperature as a result of the combined thermal motions of the nuclei. In this case the unitary matrix which defines the relation between the diabatic and adiabatic Hamiltonian matrix is the one which minimizes the difference between the transition dipole moments from the ground state of the diabatic states and the transition dipole moments from the ground state of two isolated molecules in the same geometry. By combing this diabatization scheme within a TDDFT description with classical MD, they could investigate the effects of dynamics in the excitonic coupling in molecular crystals of anthracene and tetracene. The results of such an investigation was that the excitonic coupling undergoes very large fluctuations at room temperature as a result of the combined thermal motions of the nuclei, thus significantly affecting the description of exciton transport in the organic crystals. To analyze in more detail the excitonic coupling dynamics, the Fourier transformation of the autocorrelation function of the fluctuations of the coupling has been computed (see Figure 4)
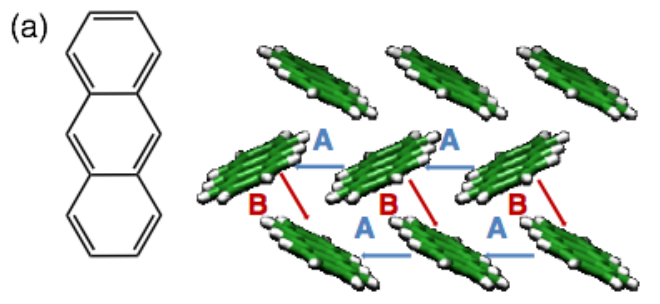

(b)

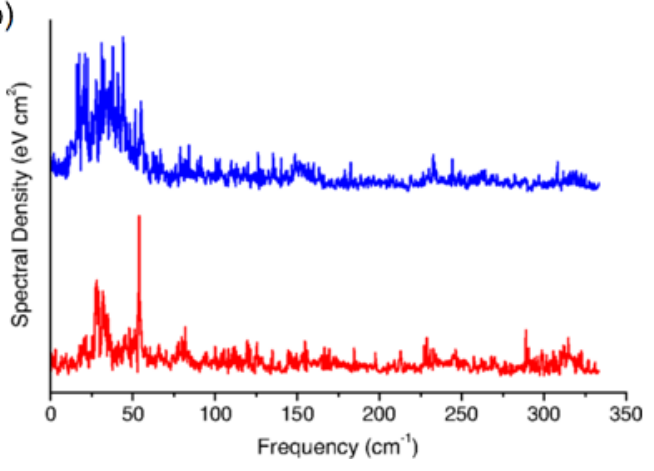

Figure 4. (a) Chemical structure and crystal arrangement for anthracene with the labeling of the nonequivalent nearest neighbors (molecular dimers A and B). (b) Fourier transform of the autocorrelation function of the excitonic coupling between the first excited states of the neighboring molecules for anthracene dimers A (top) and B (bottom), at $300 \mathrm{~K}$. Reprinted and modified with permission from Ref [310]. Copyright 2015, American Physical Society. 
The spectral density reported in Figure 4 reveals that low-frequency vibrations below 75 $\mathrm{cm}^{-1}$ for both pairs $\mathrm{A}$ and $\mathrm{B}$ in anthracene are responsible for the large modulation of the excitonic coupling whereas vibrations above $200 \mathrm{~cm}^{-1}$ seem to hardly contribute to the modulation of the coupling. The peaks in the spectral density correspond to low frequency phonon modes with the largest exciton-phonon coupling. If only the Coulombic interaction is used, the exciton coupling fluctuation is much reduced, suggesting that short-range interaction is the main player for the modulation of excitonic couplings in molecular crystals.

\subsubsection{Quantum dots and plasmonic nanoparticles}

Recently, organic-inorganic hybrid materials attracted considerable attention in applications as artificial light-harvesting systems for their low costs and excellent mechanical properties, which are required for device applications.

Many hybrid complexes incorporate colloidal semiconductor quantum dots (QDs), also known as nanocrystals (NCs) which offer several advantages over traditional organic chromophores. These include intense photoluminescence, large Stokes shift with narrow, tunable emission (based on QD diameter or composition), excellent resistance to photobleaching, and recent advances in functionalization of the QD surface. For light harvesting applications, many of these properties favor the use of QDs as the primary antenna for electromagnetic radiation. QDs are most frequently used as FRET donors paired with fluorescent dyes, dark quenchers, or fluorescent proteins as acceptors. ${ }^{286}$ The advantages of QDs as donors include the flexibility to efficiently excite the QD while minimizing direct excitation of the acceptor, the ability to optimize the spectral overlap between the QD and acceptor without introducing problematic crosstalk between their emission, and the ability to further optimize FRET efficiency by arraying multiple acceptors per QD.

Quantum-chemical methods, such as DFT, are able to describe bonding, QD-ligand interactions, and the electronic structure with a reasonable level of accuracy. Unfortunately, DFT is numerically expensive, limited to maximum QD sizes of very few $n m .^{281}$ As compared to the TDDFT treatment of organic molecules, where simulations of systems with several hundred atoms are feasible, in QDs computational cost rises due to two factors: the presence of transition metals and heavy elements (such as $\mathrm{Cd}, \mathrm{Pb}, \mathrm{Te}$ ) requiring extended basis sets and the necessity of computing many excited states to simulate the optical properties of interest. Equivalently, the modeling of NC-molecule EET requires the consideration of a huge number of excited NC states (the excitons), and every exciton couples in a different way to the molecule, resulting in a specific distance dependence of the EET. Due to these still computationally challenging aspects, simplified QM approaches (such as semiempirical tight-binding 
approaches) are commonly used to compute EET between NCs and both single molecular dyes ${ }^{311}$ or dye aggregates. ${ }^{312}$

Another critical specificity of the EET modeling involving NCs is that the distances are typically in the same range as the size of the NCs, that is, few nm: extrapolating what commonly found for $\mathrm{D} / \mathrm{A}$ molecules, the dipole approximation is expected to fail. A theoretical analysis of this issue has been reported in various works ${ }^{313-315}$ and unexpectedly it was found that the dipole approximation works surprisingly well when the donor and/or acceptor is a spherical QD, even at contact DA separations, as shown in Figure 5.

An explanation for this unexpected behavior was proposed on the bases of electrostatic considerations for DA systems made of two CdSe QDs (3.9 nm size). ${ }^{314}$ By using the semiempirical pseudopotential method to calculate the atomistic single-particle wave functions, it was shown that the transition density of the QD is built up from two quasispherical clouds with opposite sign. As a result, the potential generated by the QD transition density can be approximated as the sum of the potentials generated by two point charges located at the centers of such oppositely signed clouds. For the QD considered, such centers of charge are separated by $\sim 7 \AA$ : the QD transition density can therefore be described as a transition dipole defined by two charges separated by such distance, which is clearly smaller than the center-to-center separations accessible to donors and acceptors.
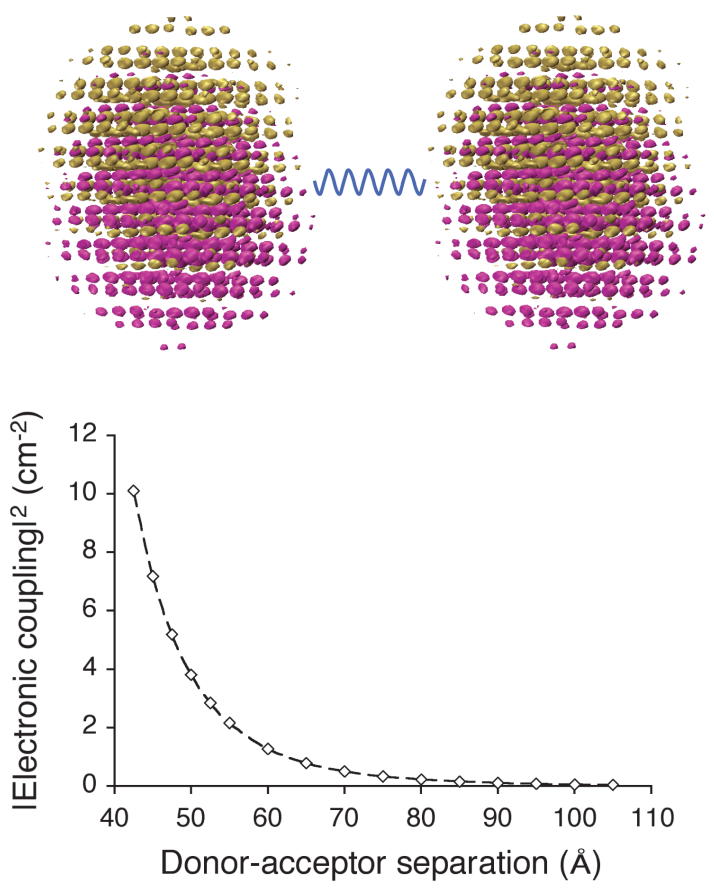

Figure 5. Squared electronic couplings predicted by the full 3D expression (diamonds) and by the point dipole approximation (dashed lines) between two $3.9 \mathrm{~nm}$ CdSe QDs as a function of their center-to-center separation. Reprinted and modified with permission from Ref $\left[{ }^{314}\right]$. 
Copyright 2008 American Chemical Society.

Energy transfer from a CdSe NC coupled to a single tetrapyrrole-type molecule has been successively studied by means of a tight-binding model combined with a CI characterization of the Coulomb-correlated electron-hole pairs. ${ }^{311}$ A replacement of the transition densities by atomic-centered partial transition charges was introduced for both the NC and the molecule. A spherical NC was compared with a similar large NC of pyramidal and hemisphere shape. Because of the different exciton energies and wave functions, the transfer rates differ somewhat for the three types of $\mathrm{NC}$, however, for all of them the Coulomb correlation essentially determines the magnitude of the transfer coupling and the exciton energy.

Still following a Coulomb scheme, the role of a complex dielectric environment (and external electric fields) in the coupling of QD has been also investigated. ${ }^{316}$ A selfconsistent solution of the Schrödinger and Poisson equations was introduced to calculate the Förster coupling between nanoparticles of arbitrary size, shape, and orientation, embedded in an arbitrarily complex electrostatic environment of positiondependent dielectric constant $\varepsilon(\mathrm{r})$. The Förster coupling was shown to be either increased or decreased as a function of the nanoparticle shape and of the properties of the dielectric environment. For example, it was shown that by inserting a third dot in between two QDs, the coupling is either suppressed or increased depending on the orientation of the material dipole moment. This can be intuitively explained by image dipoles due to the dielectric presence of the third dot. In the case of parallel dipoles perpendicular to the distance vector, the coupling is decreased because the two dots create opposite image dipoles within the third dot thereby suppressing the effective potential experienced by the other dot. In the case of parallel alignment of the dipole moments to the distance vector, the coupling increased as now, the image dipoles are aligned parallel and effectively increase the potential created by one dot seen by the other. This behavior is exactly equivalent to that expected for two chromophores (represented as dipoles) in a spherical dielectric cavity. ${ }^{132}$

Another mechanism of fundamental interest in the context of artificial LH, is the Localized surface plasmon resonance (LSPR) that exists at a surface of a metal nanostructure which is related to the collective electronic excitation of the conduction electrons in response to an applied electromagnetic field. LSPR can intensify light scattering and enhance light concentration around the nanostructure. The extreme tunability of the LSPR for noble metals nanostructures, especially those made from silver and gold upon subtle changes in size, shape, and dielectric environment, make these plasmonic nanostructures suitable for a variety of applications including surface plasmon enhanced spectroscopies.

The combination of plasmonic effects with energy transfer mechanisms has also gained a strong interest. ${ }^{317}$ 
Energy transfer between donor and acceptor fluorophores is in fact efficiently mediated through intense surface plasmon modes to extend the FRET distance range and improve the fluorescence signal. For example, it was demonstrated that long-distance energy transfer is obtained when donor and acceptor moieties are located on the opposite interfaces of an $\mathrm{Ag}$ film of $30-120 \mathrm{~nm}$ of thickness. ${ }^{318}$ Multilayer samples having a structure substrate-acceptor-Ag-donor (where the donor was a dye and the acceptor an electroluminescent conjugated polymer), have also been tested showing an enhancement of the acceptor luminescence for $\mathrm{Ag}$ thickness ranging from 30 to 150 nm. ${ }^{319}$ In both cases, the environment acts through its LSPR, which can mediate the donor-acceptor interactions. This mediating role however is extremely complex as it involves different photophysical phenomena in addition to the EET process; for example the LSPR can affect the donor absorption process, can enhance or quench the emission of both donor and acceptor moieties, and finally it can act as a competitive energy acceptor. All these SP-dependent processes are also strongly sensitive to the setup conditions, nature and shape of the metal bodies, nature and relative position/orientation of the $\mathrm{D}-\mathrm{A}$ pair, relative position/orientation between the $\mathrm{D}-\mathrm{A}$ pair and the metal, presence of a solvent, etc.

Due to this complexity, a quantum chemical description of the process is a challenge, ${ }^{320,321}$ however by combining quantum chemical descriptions of the D/A pair and an extension of the PCM described in section 2.2.1 to metal nanoparticles (MNP) an efficient strategy has been proposed. ${ }^{322}$ Two are the main aspects of novelty of this strategy if compared to others previously used:323-326 first, the molecular systems involved in the EET process, as well as the EET process itself, are not described classically in terms of (polarizable) point dipoles, but through a full QM approach; secondly the effects of the composite environment (MNPs and solvent) are described using a single QM/classical formulation in which all the components of the system (the D-A pair, the MNPs, the solvent) are mutually polarized. Moreover, the model is not limited to nanoparticles of simple shapes such as planar surfaces and spheres, and does not require assuming a dipolar-response for the metal. For excitonic systems as those represented by stacked dimers, a screening of the electronic coupling is found for all different dimer-MNP arrangements. In contrast, when donors and acceptors are separated by the MNPs, an enhancement of the electronic coupling is found together with a parallel enhancement of the EET quantum yield. However, MNPs do not only affect the excitonic interactions and the EET efficiency but they also give rise to new nonradiative decay channels that compete with the intermolecular EET process. As a result, even if the EET process is increased, the final effect is often a reduction of the emission from the acceptor.

More recently the PCM-plasmonic approach has been combined with a polarizable MM description of the molecular environment. The resulting QM/MMPol/PCM multiscale approach was applied to the modeling of the plasmon induced fluorescent enhancement of pigment-protein complexes such as $\mathrm{PCP}^{327}$ and FMO. ${ }^{328}$ The specificity of such a 
formulation is that no approximations are introduced in the modeling of the cluster of interacting pigments (which are treated quantum-mechanically) and the effect of the protein can be accounted for using a fully polarizable MM description in terms of induced dipoles (see section 2.2.1). The applications done so far have shown the potentials of this multiscale description not only in determining the optimal composition of the biohybrid device (number and type of metal nanoparticle, hotspot configurations) but also in a detailed investigation of the plasmonic effects in the different processes (absorption, energy transfer and emission) that combine to give the resulting fluorescence behavior.

A different strategy to describe MNP-induced local fields is through a microscopic theory of interacting molecule-MNP ${ }^{329}$ systems. Such a theory introduces an appropriate Hamiltonian and treats the Coulomb interaction between the molecules and the MNP nonperturbatively. Then, the complete summation of the coupling accounts for mirror charge effects and local field enhancement due to the presence of the MNP. This approach has been extended to compute the plasmon enhancement of intermolecular excitation energy transfer. The approach is based on a nonperturbative consideration of the Coulomb coupling matrix elements responsible for EET between the molecules and the MNP. ${ }^{330}$ Such a microscopic theory (i) includes the molecular electron-vibrational states in any level of sophistication, (ii) offers a microscopic description of the MNP electrons, and (iii) accounts for the molecule-MNP coupling nonperturbatively. To reduce the huge amount of electronic and vibrational degrees of freedom (DOF) a system-reservoir (environment) separation is introduced. In this approach, a separation of the MNP electron dynamics into a collective motion corresponding to that of the electrons center of mass and into an intrinsic motion relative to the center of mass motion is introduced.

\subsection{Biological light harvesting systems}

In the last decades, much research has been directed toward the development of quantitative models of light harvesting in PPCs able to rationalize their spectral and energy transfer properties based on the crystal structure. ${ }^{2-21}$ In some cases, structural information is only used to estimate the electronic couplings among the participating pigments using simple approaches like the point dipole approximation described in Section 3. Then the site energies in the complex are determined by simultaneous fitting of a variety of steady-state or time-resolved spectroscopic data. Often, like in the LHCII complex of plants, the large number of pigments involved make those fittings ambiguous, so other studies, especially in the last years, attempt also the prediction of site energies using the quantum-chemical methods described in Section 2. Due to the recent discovery of long-lasting quantum coherence effects in several photosynthetic complexes, ${ }^{183,184,188-200}$ which have underscored the importance of the vibrations in the 
energy transfer process, in the last decade some groups have also pursued the prediction of the spectral density of pigment-protein coupling from simulation (see section 4).

In the following sections, we summarize the studies on photosynthetic PPCs performed mostly in the last 20 years by using quantum chemical methods. In particular we shall follow a classification based on the location of the PPC with respect to the photosynthetic membrane and the reaction centers. Integral membrane antennas like LH2 or LHCII cross the lipid bilayer, whereas peripheral membrane antennas like the phycobilisomes of cyanobacteria, or chlorosomes and the FMO complex from green bacteria are linked to one side of the membrane. Moreover, integral membrane antennas can be divided into core antennas, closely associated with reaction centers, like the CP43 and CP47 complexes of RC PSII or the LH1 complex of purple bacteria, and accessory antennas, like LHCII or LH2.

The description of peripheral, accessory and core antennas, will be followed by that of reaction centers; in this case, the focus will be restricted to their excited states and lightharvesting properties rather than their charge transfer characteristics, which are outside the scope of the present review.

\subsubsection{LH1 and LH2}

The light-harvesting antennas of anoxygenic purple bacteria have been characterized in great detail, and they constitute probably the best understood of all antenna systems in photosynthetic organisms. It is constituted by two light-harvesting antenna complexes known as the light-harvesting 1 and 2 (LH1 and LH2). ${ }^{331,332}$ LH2, also called B800-850, is the main accessory antenna complex found in the membrane, characterized by two strong absorption bands at 800 and $850 \mathrm{~nm}$, whereas the core LH1 complex shows a single absorption band at $875 \mathrm{~nm}$ and is closely associated with the RC. In some species a spectroscopic variant of LH2 called LH3 (B800-820) is found when the organism is grown at low-light conditions. In LH3 the $850 \mathrm{~nm}$ band in LH2 is displaced to $820 \mathrm{~nm}$, and this antenna partially or even completely replaces the presence of LH2 in the membrane. ${ }^{333}$ The crystal structure of LH2 was first solved for the bacteria Rhodopseudomonas acidophila ${ }^{334-336}$ and Rhodospirillum molischianum, ${ }^{337}$ and later structures for the LH1-RC complex in Rhodopseudomonas palustris ${ }^{338}$ as well as for the LH3 antenna of Rps. acidophila ${ }^{333}$ were obtained. All three PPCs display a symmetric ring structure based on a common repetitive $\alpha-\beta$ subunit formed by two transmembrane alpha helices, the $\alpha$ - and $\beta$-aproprotein, three BChls and one carotenoid (rhodopin glucoside). In LH2, the size of the ring have ca. $65 \AA$ diameter and can vary among different species, and the crystal structures solved so far describe 8-fold (24 BChls, Rs. molischianum) and 9-fold (27 BChls, Rps. acidophila) symmetries, as illustrated in Figure 6. For LH3, the crystal structure solved contained the same 9-fold symmetry as 
LH2 in Rps. acidophila. In contrast, the LH1 ring has a larger size of ca. $120 \AA$ diameter and can contain up to 16-18 subunits, each one containing two BChls.

a)
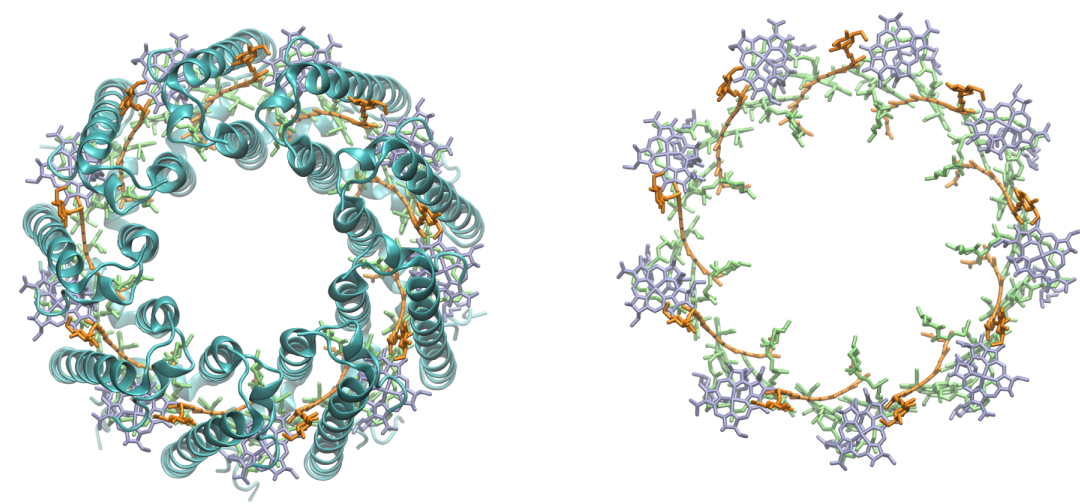

b)
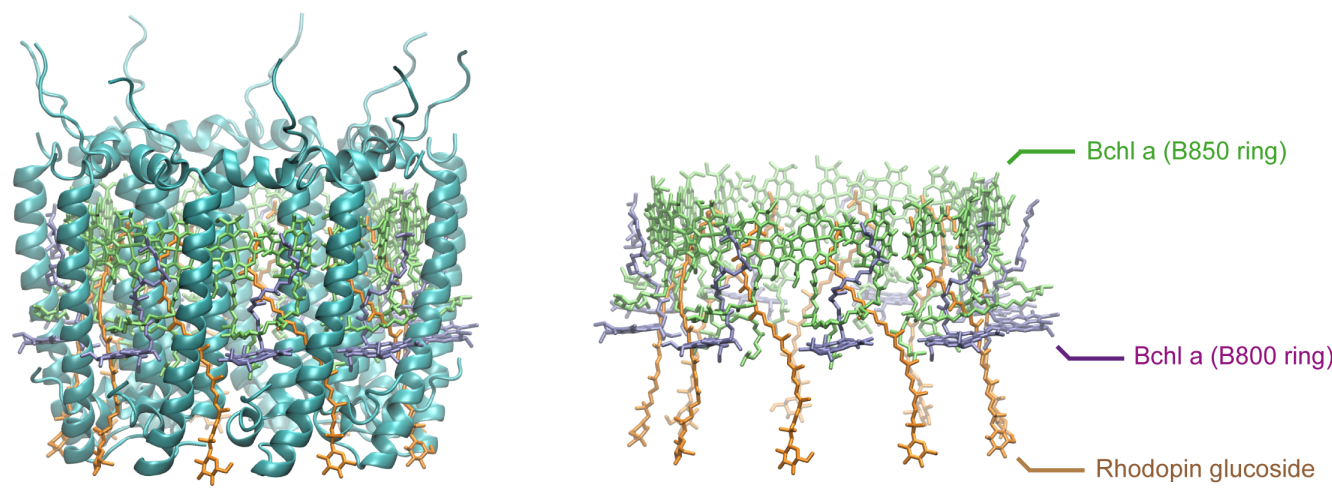

Figure 6. Structure of the LH2 antenna of the purple bacterium Rhodopseudomonas acidophila. ${ }^{339}$ a) Top view. b) Side view.

The impact of excitonic coupling on the excited-states of LH2 is immediately apparent in the two absorption bands observed at 800 and $850 \mathrm{~nm}$, and can be understood based on the double-ring arrangement of BChls in the complex. ${ }^{331,340}$ Thus, the 8-9 BChls that contribute to the $800 \mathrm{~nm}$ feature, which form a ring with the $B C h l$ planes parallel to the membrane plane, are relatively far apart from each other thus showing weak electronic couplings among them. In contrast, the 16-18 BChls that contribute to the $850 \mathrm{~nm}$ band are closely packed (ca. $9 \AA$ center-to-center separation) with their molecular plane almost perpendicular to the membrane plane. The strong electronic interactions in the B850 ring translate into a band of excitonic states, where the oscillator strength is concentrated in states absorbing near $850 \mathrm{~nm}$. Thus, the LH2 antenna is an elegant example on how absorption spectra can be tuned through excitonic interactions, although the axial ligands and the amino acids hydrogen-bonded to the $B C h l$ also modulate the site energies of the pigments, and therefore their optical spectra. ${ }^{248,341-343}$

The strong dependence of the spectral and energy transfer properties of LH2 on the particular values of electronic couplings involving the $B C h l$ pigments in the B850 ring has made it a perfect test system for progressively more and more accurate quantum 
chemical calculations of this important quantity (see section 3.1). Thus, the first calculations based on the crystal structure of LH2 adopted transition-monopole expressions evaluated using the semiempirical QM-consistent-force-field/ $\pi$-electron (QCFF/PI) method, ${ }^{344,345}$ or mixed strategies where interactions among close pigments were derived from supermolecule semiempirical INDO/S-CI calculations, whereas couplings involving well-separated BChls where estimated using the dipole approximation. ${ }^{346-348}$ The coupling strengths estimated in such studies, which allowed rationalizing the spectra of $\mathrm{LH} 2$, clearly indicated a strong delocalization of the excitedstates in the B850 ring. At the same time, it also became evident that static disorder could play an important role on the light harvesting process by redistributing oscillator strength among the exciton states. ${ }^{345,346}$ Despite the absence of a crystal structure for LH1, a geometric model of the LH1-RC assembly was also modeled by the Schulten group, allowing a first estimate of the electronic coupling strengths among pigments of the LH1 antenna and the RC. ${ }^{346}$ Subsequent advances based on the ab initio CIS method allowed the calculation of the Coulomb contribution to the couplings in LH2 through the introduction of the TDC method by Krueger et al. ${ }^{122,349,350}$ This methodology allowed a more realistic account of the 3D shape of interacting transition densities, and indicated that the PDA leads to strong errors in the evaluation of the Coulomb coupling at distances less than $15 \AA$ for $B C h l s$, and even at distances longer than $20 \AA$ for elongated molecules like carotenoids. ${ }^{122}$ Those studies also suggested that the carotenoids in LH2 could mediate the $\mathrm{B} 850 \rightarrow \mathrm{B} 800 \mathrm{BChl}-\mathrm{BChl}$ transfer through superexchange interactions, thus potentially explaining the apparent discrepancy between observed rates and those predicted by Förster theory. ${ }^{350}$ Moreover, the TDC method was used to explore the coupling strength between the $\mathrm{S}_{2}$ (bright) and $\mathrm{S}_{1}$ (dark) states of carotenoids to the $\mathrm{Q}_{\mathrm{x}}$ and $\mathrm{Q}_{\mathrm{y}}$ states of $B C h l$ in $\mathrm{LH} 2$, an interaction that would be zero by definition based on a point dipole picture. That study indicated that the major light harvesting pathway involving carotenoid absorption in LH2 should involve an $\mathrm{S}_{2} \rightarrow \mathrm{Q}_{\mathrm{x}}$ energy ransfer, ${ }^{349}$ a conclusion supported by later calculations performed by the Damjanovic and co-workers based on the PPP Hamiltonian. ${ }^{33}$ Interestingly, that study addressed for the first time both singlet and triplet transfers in LH2 by evaluating the full Coulomb and exchange coupling integrals, thus showing that triplet states in 8 $B C h l s$ of the B850 ring could be efficiently quenched by the carotenoids.

Later studies used QM calculations to provide further insights in the electronic interactions in LH2. For example, calculations including nearby amino acids were used to assess the impact of the environment in the couplings, ${ }^{351,352}$ and comparison of supermolecule and TDC calculations showed that short-range contributions could account for $20 \%$ of the interactions in LH2. ${ }^{341}$ Calculations based on the CEO approach by Tretiak and co-workers explored the impact of dielectric environment effects on the excitonic properties as well as the validity of the exciton model in LH2, and underscored the importance of the predicted transition dipole strength for a given QM model chemistry in order to obtain reliable coupling estimates. ${ }^{353,354}$ A series of studies further examined the role of the carotenoid in the complex, and showed that $S_{1}-Q_{x}$ 
couplings involving the $B C h l$-carotenoid pair could be approximated by scaling the corresponding $\mathrm{S}_{2}-\mathrm{Q}_{\mathrm{y}}$ ones, given that the $\mathrm{S}_{1}$ state gets oscillator strength by mixing to the $\mathrm{S}_{2}$ state. ${ }^{123,355-357}$

Overall, these studies underscored the several aspects that should be considered in order to obtain a reliable QM estimate of electronic couplings in systems where the pigments are closely spaced: 1) the need to account for the 3D shape of the transition densities in the prediction of Coulomb contributions, 2) the inclusion of short-range effects and 3) the eventual correction of the computed couplings by scaling techniques if the transition dipole moment of the pigment is not well described by the QM method of choice. Apart from these important insights, the modeling of the energy transfer rates among the B800 localized and the B850 excitonic states motivated the development of generalized Förster theories, where incoherent hoppings among multichromophoric donor and acceptor states are described. 358,359

Because the spectral features in LH2 are mostly determined by excitonic coupling and static disorder, and thanks to the symmetry of the $B C h l$ molecules in the complex, the site energies of the 3 distinct $B C h l s$ where often taken as adjustable parameters in the studies described above. Several groups however studied how the individual site energies of the $\mathrm{B} 800$ and $\mathrm{B} 850 \mathrm{BChl}$ are tuned by the environment. TDDFT calculations, for example, showed that the similar red shift observed in the B800 BChl a molecules of Rs. molischianum and Rps. acidophila arise in one case due to the axial ligand coordinating the $B C h l$, whereas in the other case the shift originates mostly from a hydrogen-bond. ${ }^{342}$ Later studies based on a combined QM/MD strategy showed that the spectral broadening in the B800 band arises from the polar environment, whereas in the B850 band it is due to excitonic effects. ${ }^{247,248}$ These studies also provided the first prediction of the spectral density of pigment-protein coupling for a pigment embedded in a PPC. Further investigations, however, showed that QM/MM calculations performed either at the ZINDO, CIS or TD-DFT level could lead to quite different site energy fluctuations. Interestingly, though, they suggested that correlations among the energy fluctuations of different $B C h l s$ in LH2 should be negligible. ${ }^{124,360}$ A recent study combining MD simulations and electrostatic shift calculations provided the first simulation of the 2D electronic spectra of LH2 from atomistic simulations, and suggested that the B850 to B800 energy transfer is mediated by intermediate states delocalized over both rings. ${ }^{361}$

Concerning the detailed characterization of the site energies in LH2, it is worth noting the subsystem TD-DFT calculations performed by Neugebauer, ${ }^{343}$ which allowed for the first time to account for large portions of the protein environment at a DFT level. That study in addition provided coupling constants in good agreement with previous empirically-scaled CIS estimates. Semiempirical ZINDO calculations were later presented to study the spectral properties of the wild type and a mutant LH2 and LH3 
antennae of Rhodobacter sphaeroides, suggesting that excited-state absorption is dominated by charge transfer states between adjacent chromophores. ${ }^{362}$ A detailed analysis of the site energies in the LH2 and LH3 complexes was also provided in two recent studies, in which the protonation pattern along the complex was used as a tunable parameter in electrostatic shift calculations in order to describe the spectra of the complexes. Interestingly, those studies showed that in order to explain the spectral changes between LH2 and LH3 not only the site energies of the B850 (B820) ring should be changed, but also a 2-fold decrease in B820 couplings should be taken into account. ${ }^{363,364}$

It is also worth noting the studies performed by the Schulten group, where atomistic simulations on the largest photosynthetic assemblies performed so far, including multiple LH1-RC and LH2 complexes, where performed in order to assess the impact that the spherical chromatophores of Rhodobacter sphaeroides ${ }^{365}$ or the lamellar chromatophore from Rhodospirillum photometricum ${ }^{366}$ have on the overall light harvesting process. Other recent studies have allowed, for example, determination of the DFT molecular orbitals of the complete LH1-RC and LH2 complexes using the EBFMO fragmentation method, ${ }^{367}$ or understanding why the natural sizes for LH2 rings are indeed optimized for light harvesting from combined MD and TD-DFT calculations. ${ }^{368}$

\subsubsection{Chlorosome and FMO}

The photosynthetic apparatus of green sulfur bacteria is characterized by the largest light-harvesting antenna system found in nature, the chlorosome, which contains hundreds of thousands of $B C h l$ pigments inside a lipid monolayer. ${ }^{369}$ The detailed structure of this huge complex is unknown due to its intrinsic large structural disorder. However, a model of the chlorosome structure has recently been obtained by combining solid-state NMR and cryo-EM images of a mutant bacterium that synthetizes a chlorosome mostly containing BChl $d$ and forming regularly packed elements. ${ }^{370}$ The chlorosome is connected to the reaction center by the water-soluble Fenna-MatthewsOlson (FMO) complex. ${ }^{371,372}$ This connection, however, is also mediated by the baseplate separating the chlorosome and the FMO complex, formed by CsmA proteins with a still unknown particular structural arrangement. As already said, the structure of the FMO complex, in contrast, was solved 40 years ago, representing the first structure of a light-harvesting antenna known with atomic detail. ${ }^{1,373}$ That structure revealed the precise disposition of seven $B C h l$ a molecules contained in each monomer of the overall homotrimer structure of the complex. However, recent crystallographic studies on the FMO complex of Prosthecochloris aestuarii and Chlorobaculum tepidum have revealed the presence of an additional eight $B C h l$ a molecule. ${ }^{374}$ 
a)

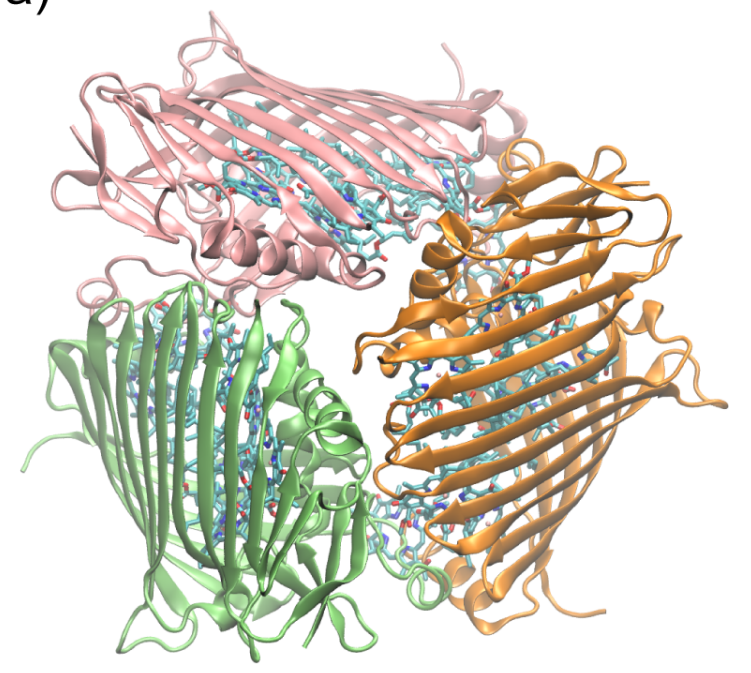

b)

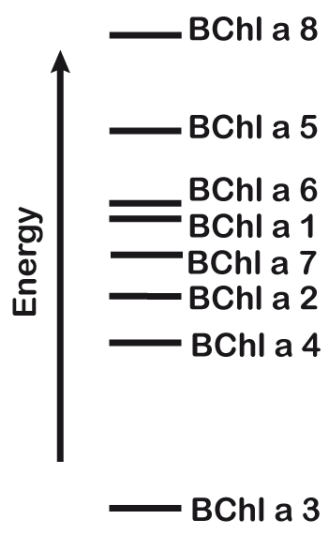

c)
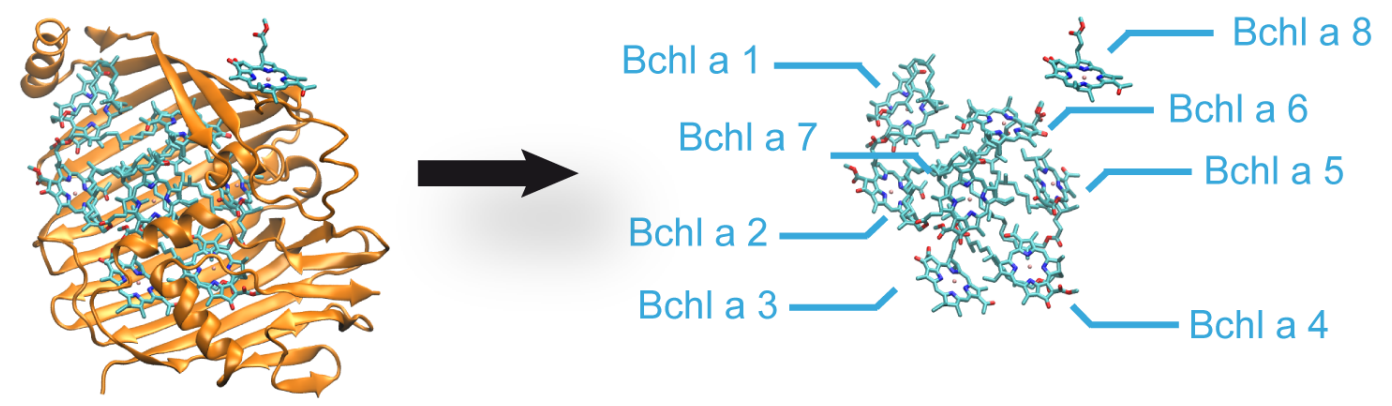

Figure 7. a) Trimeric structure of the FMO complex of the green sulfur bacterium Prosthecochloris aestuarii. ${ }^{374}$ b) Structure-based calculations estimate a site energy range of $\sim 500 \mathrm{~cm}^{-1}$ caused by pigment-protein interactions. ${ }^{375} \mathrm{c}$ ) Arrangement of the eight $B C h l a$ pigments in the monomer unit of FMO.

Since the first structure of FMO was solved, a large number of theoretical and experimental studies have addressed the relation among three-dimensional structure and the optical and energy transfer properties of the complex. In contrast to the LH2 antenna of purple bacteria described in the previous section, where the main features of optical spectra arise due to strong exciton interactions in the B850 ring, the spectral features in FMO arise from the subtle tuning of the individual site energies due to the surrounding protein environment. Thus, whereas LH2 represents an ideal system in order to critically assess the reliability of electronic couplings estimated from QM methods, a large number of studies have tried to determine the relative ordering of the site energies in the FMO complex from structure-based calculations, a problem which has turned to be considerably more challenging than the prediction of couplings. This was clear from an initial study on the spectral properties of FMO, in which the assumption of common site energies for all $B C h l s$, as was similarly done for LH2, was unable to explain its 
spectral features just from the impact of excitonic interactions. ${ }^{376}$ The first study addressing this problem in the early 1990s used ZINDO semiempirical calculations of the $B C h l$ excited states including the presence of axial ligands and charged residues at distances less than $5 \AA$ from the $B C h l s .{ }^{377}$ Whereas the predicted site energies still lead to unsatisfactory spectra, that study underscored the leading role of site energy shifts due to conformational effects or pigment-protein interactions compared to the shifts exherted by excitonic couplings in FMO.

Adolphs and Renger achieved the first agreement between predicted and experimental spectral features in 2006, about 30 years after the first crystal structure of FMO was solved, by including the effect of all charged amino acids in the complex in electrostatic shifts calculations of the site energies based on the changes in dipole moment between ground state and the $\mathrm{Q}_{\mathrm{y}}$ excited state of $B C h l .{ }^{378}$ That study also allowed rationalization of the small magnitude adopted for the $\mathrm{Q}_{\mathrm{y}}$ transition dipole moment in empirical fittings of the FMO Hamiltonian from the impact of dielectric screening effects in the electronic couplings. In that study, $B C h l-3$ was predicted to be the energy sink of the system, suggesting that FMO should be oriented with $B C h l-3$ in close proximity to the RC, a prediction later confirmed by experiments. ${ }^{379}$ These calculations were later refined in a more elaborate approach including the electrostatic effect of all amino acids in the complex, a non-standard protonation pattern in the complex, a more realistic description of the charge distribution in the electrostatic shift calculations, and polarization effects due to the surrounding dielectric environment. ${ }^{85}$ These studies suggested that the major features in the FMO spectra arise from electrostatic pigment-protein interactions, and in particular, mainly from the impact of the backbone of two alpha helices. Because conformational effects were limited to the impact of the torsion angle of the acetyl group on the $B C h l$ energies, the agreement found with experimental spectra suggested that out-of-plane bendings should have small effects $<100 \mathrm{~cm}^{-1}$.

Later a simpler but still effective recipe to compute site energies based on electrostatic shifts (see section 2.2.2) was also proposed, suggesting that the assumption of a standard protonation pattern for the FMO protein leads to a broader spectrum, the largest $B C h l$ energy difference being $495 \mathrm{~cm}^{-1}$ instead of 425 as predicted by the nonstandard protonation pattern. ${ }^{86}$ Inclusion of the eight $B C h l$ recently discovered in FMO in these calculations indicated that such $B C h l-8$ should be the highest-energy site in the complex, suggesting that it acts as the linker to the baseplate, as shown in Figure $7 .{ }^{375} \mathrm{In}$ that study, in addition, the impact of the neighboring baseplate and $\mathrm{RC}$ dielectric environments on the protonation pattern of FMO was predicted to lower the $B C h l-8$ energy by $80 \mathrm{~cm}^{-1}$. Different empirical fits of the site energies in FMO, however, lead to a variety of sets which can still describe its optical properties, ${ }^{372}$ thus the agreement with several experimental spectra can not be taken as a definitive proof of the goodness of the predicted sets of site energies. For example, ZINDO QM/MM calculations performed along MD trajectories of the FMO complex by different research groups lead to contradictory positions for $B C h l-8,{ }^{380,381}$ one of the studies however supporting its position as the highest-energy site in the complex. ${ }^{375,381}$ Interestingly, the $B C h l-8$ 
pigment was found to spontaneously unbind FMO monomers during MD simulations, ${ }^{380}$ suggesting it could be only partially bound to the complex in experiments performed on the monomer.

The still considerable challenge in accurate prediction of site energies relies on the many aspects that can impact the final results. Some groups follow a strategy based on the crystal structure and mostly neglecting the impact of $B C h l$ conformation on site energies, like the studies performed by Renger and co-workers, ${ }^{85,86,375,378}$ whereas other groups account for thermal effects by averaging the computed properties over classical MD simulations. ${ }^{380,381}$ If internal geometries are not accurate enough as described by the classical force field, however, such strategy can also lead to substantial errors. On the other hand, the classical point charge description of the environment has been shown to introduce differences up to $100 \mathrm{~cm}^{-1}$ in site energies compared to linear-scaling DFT calculations of FMO in which the protein environment is described at the QM level. ${ }^{382}$ In that strategy, different calculations are performed for each $B C h l$ in the presence of the environment, and electronic couplings are later computed from the corresponding transition densities. Another interesting strategy allowed determination of the uncoupled excited states of the whole complex from a single calculation based on subsystem TDDFT, which also allows extracting the coupling constants by turning on the coupling among different subsystems. ${ }^{99}$ That strategy has also been extended to allow the separation of bonded units among different subsystems. ${ }^{104}$ In these strategies, however, it is not straightforward to include protein dielectric screening effects in the estimated couplings, which can substantially impact the interpigment interactions. In subsystem TD-DFT, this can be done by activating the couplings among the pigment excited states and those from the environment, but this is at present rather costly. ${ }^{142}$ Another strategy to improve the point-charge description of the environment relies on QM/MM schemes based on polarizable force fields. A unique advantage of this approach is that it allows to account for the impact that a heterogeneous polarizable environment has on excitonic couplings, an effect that has been shown to modulate quite differently the interactions between different pigment pairs, leading to effective dielectric constants in the range 0.9-2.3 for the FMO complex, significantly different from the common assumed value of $2 .{ }^{137}$ Finally, benchmark studies on FMO have shown the rather considerable impact that the adoption of different QM approaches, like ZINDO, CIS, TD-DFT, RI-CC2 and DFT/MRCI, can have on the predicted sets of site energies. ${ }^{51}$

Beyond the challenging determination of site energies, the observation of long-lived coherences in the FMO complex at both low and room temperature ${ }^{183,383,384}$ has also spurred a plethora of studies aimed at understanding the impact that site energy fluctuations have on exciton dynamics. As we describe in Section 4, the objective of such studies has been, first, to assess the significance of correlated fluctuations of site energies, ${ }^{226,227,230}$ and second to characterize the structured nature of the spectral density of electronic-vibrational coupling and its impact on coherent energy transfer. ${ }^{227,230,252,257,258,380,385}$ Regarding the significance of site energy correlations, results based on MD-QM/MM strategies so far predict those to be negligible. ${ }^{226,227,229}$ 
Some cross-correlations were observed involving site energies and couplings estimated using the dipole approximation, however, those disappeared when the more accurate TrESP method was adopted. ${ }^{226}$

However, the limited simulation time in those studies precludes assessing the impact of very slow protein motions. Interestingly, a recent study based on a classical normal mode analysis of the whole FMO motions showed that such slow modes can indeed induce significant correlations in site energies, although such effects were found to have a minor impact on exciton population dynamics and dephasing of coherences. ${ }^{230}$

Regarding the determination of spectral densities, a first prediction was attempted by the Kleinekathöfer group based on ZINDO QM/MM calculations performed over an MD trajectory, showing that the different $B C h l$ pigments in FMO experience a rather similar spectral density. ${ }^{252}$ Such calculations allowed describing exciton dynamics and linear and two-dimensional spectra of the FMO complex from a time-dependent exciton Hamiltonian, indicating that coherent oscillations show be larger in the FMO trimer compared to the monomer, although those were found to be small at room temperature. ${ }^{380}$ A later study based on open quantum system approaches and TD-DFT QM/MM calculations, performed again along classical MD trajectories, found coherent beatings lasting for $400 \mathrm{fs}$ at $77 \mathrm{~K}$ and $200 \mathrm{fs}$ at $300 \mathrm{~K}, 227$ in agreement with experiments, despite the absence again of site energy correlations. ${ }^{183,383,384}$ An alternative strategy based on a semiclassical simulation of exciton transfer in FMO allowed incorporation of protein relaxation effects in excited-state non-adiabatic simulations, effects neglected in standard ground state MD simulations. ${ }^{253}$ In this case, ground and excited state energies were obtained from the potential energy of the classical force field parameterized both for the GS and $\mathrm{S}_{1}$ states. The results indicated that pigment vibrations tend to diminish coherence effects at the ensemble level, although such effects were found to be much larger at the single-complex level. The study also remarked the importance of coupling fluctuations in coherent energy transfer. Further studies examined, for instance, the impact that the water-glycerol mixture adopted in experiments has on the spectral density as compared to a water solution, ${ }^{258}$ or showed that water-accessible pigments can experience dissipative environmental interactions much larger than pigments buried inside protein. ${ }^{257}$ A very recent study, on the other hand, parameterized the effect due to the pigment vibrations based on ab initio DFT molecular dynamics simulations of the pigments in gas phase. ${ }^{385}$

The above-mentioned studies allow prediction of the spectral densities at frequencies over $\sim 100 \mathrm{~cm}-1$, as the spectral densities are typically derived from $B C h l$ autocorrelation functions computed for trajectories in the picosecond timescale. The impact of very low frequencies would need to extend MD simulations to much larger timescales. An interesting alternative was proposed by Renger and co-workers based on a classical normal mode analysis of protein motions. ${ }^{230}$ Assessment of the coupling of site energies along the normal modes allowed prediction of the low-frequency features of the FMO spectral density, showing good agreement with experiments. 
Recently, the Aspuru-Guzik group has also presented a series of detailed studies on the light harvesting processes in the chlorosome, ${ }^{260,386,387}$ based on the model structure recently proposed, ${ }^{370}$ by combining TD-DFT calculations and MD simulations in order to derive the excitonic Hamiltonian and the spectral density of the system. Then, a subsequent study has allowed modeling the whole photosynthetic unit of green sulfur bacteria including the chlorosome, the baseplate, the FMO complex and the RC. ${ }^{388}$

\subsubsection{Phycobilisome and phycobiliproteins}

Compared to land plants, the available light that algae can harvest in marine environments is significantly reduced. For that reason, marine photosynthesis often uses special antenna complexes called phycobiliproteins (PBP) able to harvest sunlight in the 450-640 nm spectrum, where chlorophyll absorption is minimal. Such PBP complexes contain special linear tetrapyrrole pigments called bilins, which are covalently attached to the protein scaffold through single or double cysteine bonds. The remarkable variety of bilin types then allows algae to adapt the spectral cross-section of their antenna complexes to the particular needs in a given habitat. In cyanobacteria and red algae, PBPs are organized in large multiprotein complexes anchored to the stromal face of the thylakoid membrane called phycobilisomes (PBS), ${ }^{389}$ which can contain different types of PBPs, namely, phycoerythrin (PE), phycoerythrocyanin (PEC), phycocyanin (PC) and allophycocyanin (APC). Such PBPs are organized as $(\alpha \beta)_{3}$ trimers of a basic $\alpha \beta$ monomer unit. In cryptophyte algae, however, the organization of the photosynthetic machinery is substantially different. These algae display a greater spectral diversity, although each species contains a single PE or PC antenna protein. In addition, such PBPs are located in the thylakoid lumen and are organized as an $\alpha_{1} \alpha_{2} \beta \beta$ dimer.

Since the first structures of PBPs were solved, a variety of experimental and theoretical studies have attempted to rationalize their spectral and energy transfer properties in terms of the underlying pigment-protein structure. In the LH2 and FMO complexes described in previous sections, exciton coupling or pigment-protein interactions mainly dictate the features of their absorption spectra, respectively, as they contain a single type of chlorophyll pigment. In PBPs, however, different types of bilins can be found in the same complex, and the possibility to tune their protonation states or their rather flexible conformation by the protein environment provides further mechanisms to adapt the spectral cross-section for light-harvesting. Scharnagl and Schneider performed the first investigation on the relation among structure and light-harvesting properties of a PBP based on QM calculations by studying the impact that interactions with nearby residues had on the excited state properties of the three phycocyanobilin (PCB) pigments in the $\alpha$ - and -subunits of C-phycocyanin (C-PC) from the cyanobacterium Mastigocladus laminosus. ${ }^{390,391}$ A subsequent study further investigated the properties of the phycoviolobilin (PVB) chromophore in the $\alpha$-subunit of phycoerythrocyanin (PEC) from the same cyanobacterium by combining semiempirical ZINDO calculations with MD simulations. ${ }^{392}$ In those studies, it was assumed that all pyrrole rings in the 
pigments are protonated, as suggested by a coordinating Asp residue found in the crystal structures. This was later supported by Kikuchi and co-workers from ZINDO calculations performed on the PCBs of the C-PC complex isolated from the cyanobacterium Fremyella diplosifhone, which confirmed the protonated state of the bilin pigments by comparison to experimental spectra. ${ }^{393}$ Further studies based on TDDFT calculations coupled to the PCM solvation model, but explicitly including the effect of the coordinating Asp residue, further supported the protonated state of the pigments in C-PC and allowed the rationalization of its spectral and energy transfer properties in terms of the underlying structure, ${ }^{394,395}$ as well as underscored the large impact that conformational flexibility can have on the PCB excited state properties. ${ }^{396}$ Womick and co-workers later used spectroscopic experiments to develop a model for the energy transfer properties of other PBPs, in which QM calculations were used in order to derive transition dipoles moments for coupling calculations in the R-PE complex, ${ }^{397}$ or to study the impact of vibronic non-Condon effects in the spectroscopy and dynamics of C-PC by evaluating the impact of vibrational modes on QM calculated transition dipoles. ${ }^{398}$ A more recent theoretical study allowed elucidation of the energy transfer pathways in trimeric and hexameric aggregation states of the cyanobacterial CPC based on TD-DFT, CIS and ZINDO calculations. ${ }^{399}$

Regarding cryptophyte light-harvesting antennae, the first study relating lightharvesting properties to the underlying structure of the complex was performed by Doust and co-workers, in a study that presented the first high-resolution structure of a cryptophyte PBP, the PE545 complex from Rhodomonas CS24. That study showed that both ZINDO, CIS and TD-DFT calculations in vacuum were able to correctly predict the two 15,16-dihydrobiliverdins (DBV) as the lowest energy pigments, although the relative order of the other six phycoerythrobilins (PEB) varied depending on QM method used. ${ }^{400}$ In a subsequent study, the PE545 excitonic Hamiltonian and energy transfer model was refined by computing excitonic couplings using the TDC method using CIS transition densities, and it was also extended to the PC645 complex of the alga Chroomonas CCMP270, which structure was also determined showing the precise location of the two phycocyanins (PCB) and the dihyrobiliverdin (DBV, b50/b61) bound on each $\beta$-subunit as well as the mesobiliverdin (MBV) pigment bound on each $\alpha$-subunit. ${ }^{401}$ Later, the electronic coupling values in PE545 and PC645 were improved by taking into account the impact of dielectric screening effects as predicted from the PCM continuum solvation model. ${ }^{133,134}$ Those couplings were first used to refine the modeling of the light-harvesting dynamics in PC645 by combining experiments and generalized Förster theory, ${ }^{402}$ and later used to establish a model for the site energies and energy transfer pathways in PE545 by combining experiments and modified Redfield theory. ${ }^{238}$ A problem in these models, however, was that several models of site energies were able to explain steady-state spectra and energy transfer kinetics observed through transient absorption experiments. This problem was addressed in later studies, where a consensus model for the site energies of PE545 was established by combining spectral modeling and CIS polarizable QM/MM calculations performed along an MD 
simulation. ${ }^{136,403}$ Interestingly, such consensus model based on experimental spectra and structure-based calculations indicated rather similar energies for the pseudo-symmetric

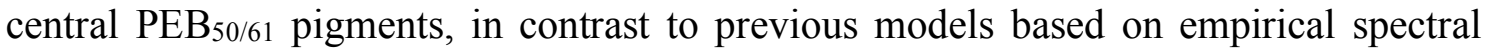
fittings, as shown in Figure 8. In contrast to chlorophyll-binding complexes, the results in these studies suggested that site energy tuning in PBPs is achieved mainly by constraining the conformation of the bilin pigments in the protein scaffold rather than through electrostatic pigment-protein interactions. In addition, the development of such polarizable QM/MM model allowed a detailed analysis on the impact that the heterogeneous polarizable environment surrounding the pigments in PE545 has on electronic couplings and energy transfer rates. The results showed that the heterogeneous dielectric properties of the complex can tune by a factor up to $\sim 4$ the energy migration rates compared to the common assumption of a homogeneous dielectric environment. ${ }^{136}$ Another study showed that exciton delocalization can considerably impact interprotein energy transfer among densely packed PE545 proteins, as found in the thylakoid lumen. ${ }^{404}$

a)
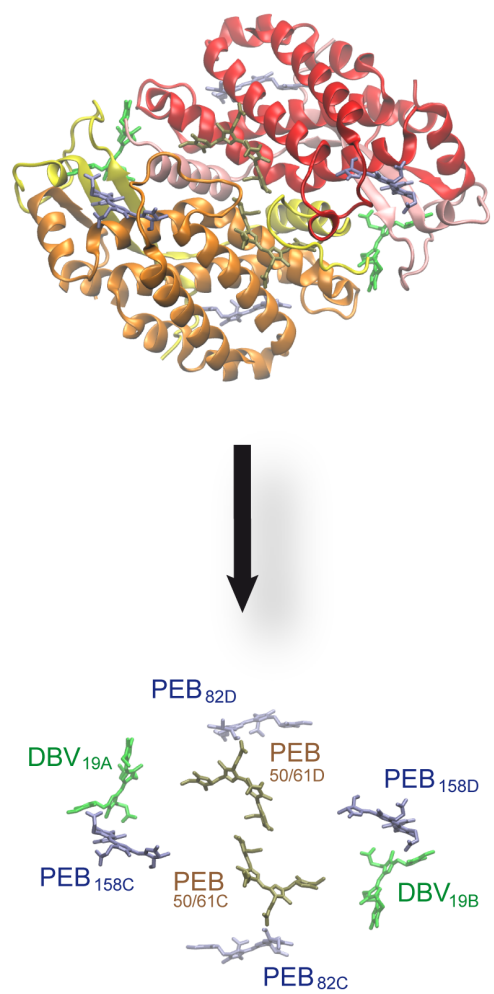

b)

Fitted site energies (several possible models)

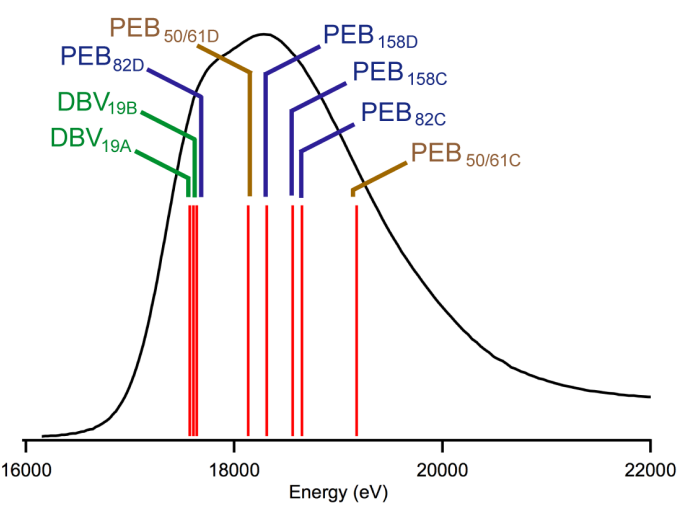

Consensus model based on QM/MMpol energies

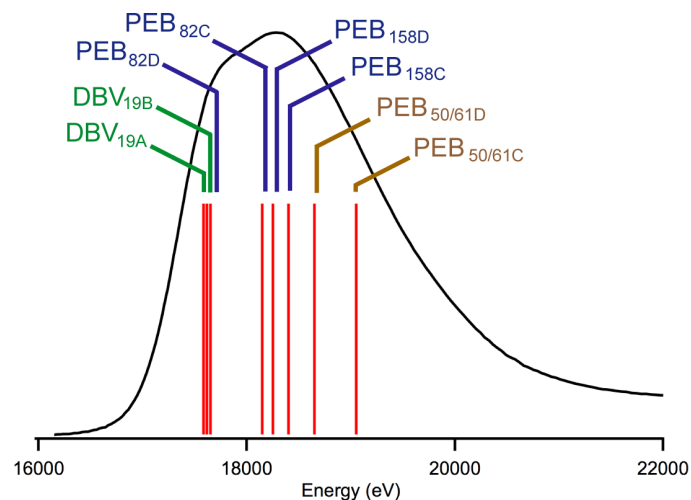

Figure 8. a) Structure of the PE545 principal antenna of cryptophyte alga Rhodomonas CS24. 400 b) Absorption spectra of PE545 and approximate position of the site energies of the eight bilins as determined by an empirical spectral fitting ${ }^{238}$ and by structure-based polarizable QM/MM calculations. ${ }^{403}$ Reprinted and modified with permission from Ref. $\left[{ }^{403}\right]$. Copyright 2013 American Chemical Society. 
In 2010, for the first time long-lived coherences were observed at room temperature for the PC645 and PE545 antenna complexes by Collini and co-workers. ${ }^{188}$ That finding lead to an increased interest in the interrelation among protein vibrations and energy transfer dynamics in those complexes. Viani et al. studied the significance of correlated fluctuations in PE545 from combined MD-QM/MM calculations, ${ }^{228}$ suggesting negligible site energy correlations that could eventually explain the persistence of the coherence effects observed. Interestingly, though, significant cross-correlations among site energies and couplings were observed, in contrast to what found previously for the FMO complex, probably owing to the more flexible nature of bilin pigments compared to $B C h l$. Such correlations have also been suggested to play a role in coherences and energy transfer rates. ${ }^{207,208,219}$ The same MD-QM/MM strategy was later used to predict the spectral densities of PE545, suggesting that individual spectral densities can considerably vary among the complex, especially for the DBV pigments, which display an overall larger coupling at low frequencies compared to PEBs. This suggests that the "structured" nature of the spectral density and its impact in coherence can be obscured if they are averaged out over the pigments in the complex, as often happens in the experimental derivation of spectral densities. In addition, the coupling among protein and pigment motions was shown to considerably impact the intramolecular vibrational modes of the pigments in the protein environment, and thus, the spectral density. ${ }^{261}$ Another interesting study relying on MD-QM/MM calculations compared the spectral densities for the PE545 and FMO complexes, showing that low frequency features in the spectral densities of FMO BChls are mostly due to environmental fluctuations, whereas in PE545 bilins they arise due to intramolecular modes. ${ }^{259,405}$ Finally, the recent determination of the crystal structures of the PC612 and PE555 complexes isolated from different algal species has shown a dramatically different quaternary structure, where the two $\alpha \beta$ monomers are rotated by $\sim 73^{\circ}$ to an open configuration compared to the "closed" configuration in other cryptophyte PBPs like PC645 and PE545, a change induced by the steric effects exerted by a single amino acid. ${ }^{200}$ Rather interestingly, experiments and CIS TDC coupling calculations showed that such "open" conformation leads to a dramatic decrease of excitonic coupling between the two central chromophores and leads to the suppression of the electronic and vibrational coherences observed in the "closed" forms. ${ }^{200}$

\subsubsection{PCP and FCP}

To capture the sunlight available in marine environments, some algae use special lightharvesting antennae containing carotenoids instead of bilins. Diatoms, which are responsible for up to $25 \%$ of total carbon fixation, ${ }^{406}$ contain the membrane-bound fucoxanthin chlorophyll-a/c protein (FCP), which shares some structural and sequence homology with the plant LHCII complex, although it carries a larger number of carotenoids per chlorophyll. ${ }^{407}$ Fucoxanthin is able to absorb blue-green light, which is 
able to reach the longest distances in water. Several techniques have been used to characterize the light harvesting properties of FCP, including two-dimensional spectroscopy. ${ }^{408-414}$ However, the absence of a three-dimensional structure of FCP has precluded structure-based calculations on this complex, although quantum chemistry has been used either to characterize the excited-states of fucoxanthin ${ }^{415}$ or its arrangement in FCP by comparison to LHCII. ${ }^{416}$

a)

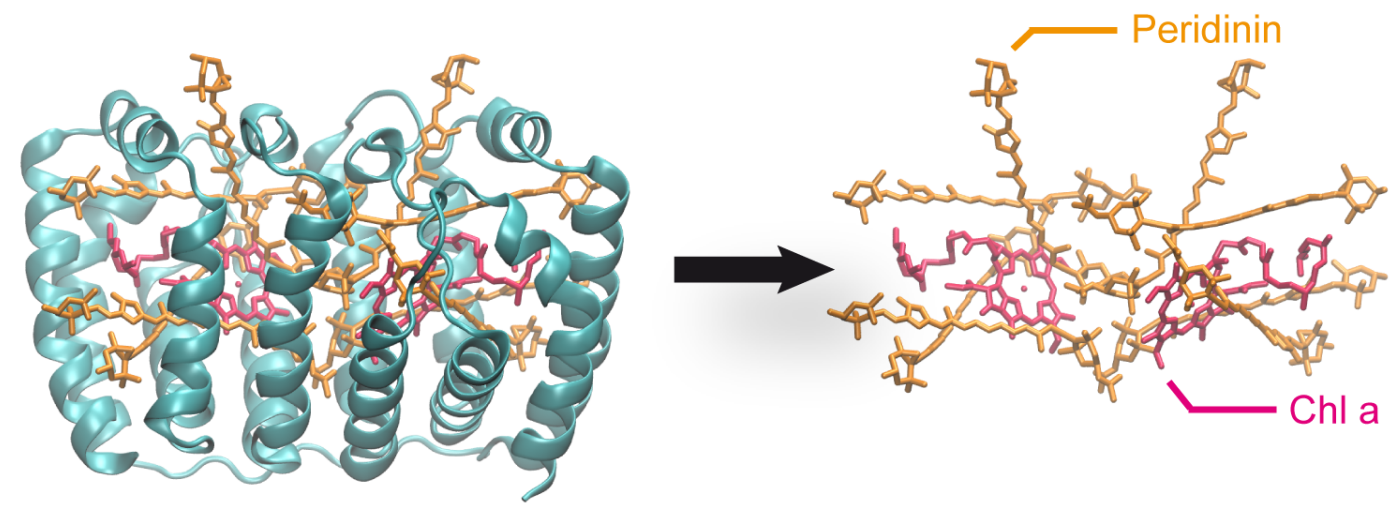

b)

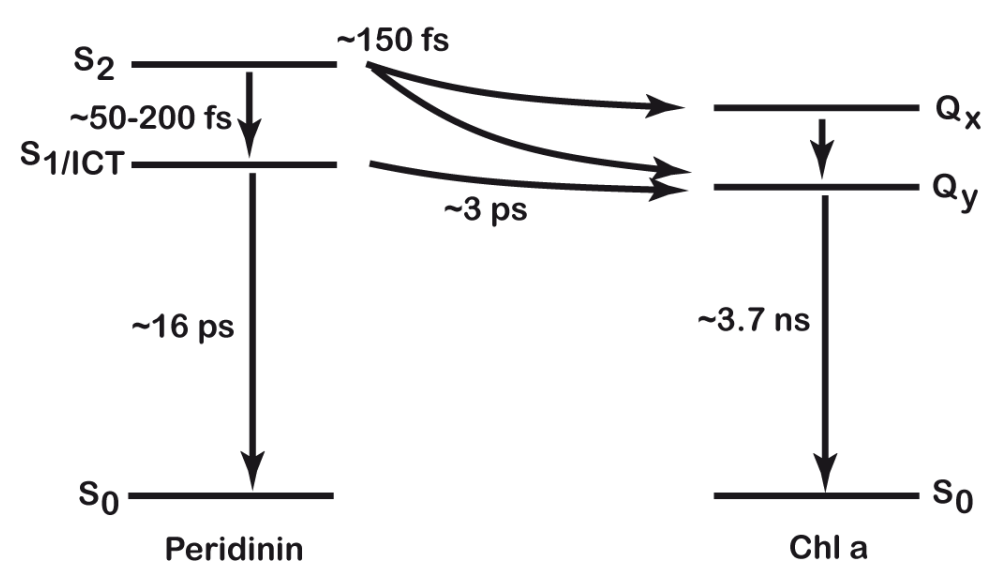

Figure 9. a) Structure of the peridinin chlorophyll-a protein monomer of dynoflagellate Amphidinium carterae. ${ }^{417}$ b) Scheme of excited states and energy transfer pathways in the complex. $^{418-420}$

Many dynoflagellates, in contrast, contain the water-soluble peridinin chlorophyll-a protein (PCP) located in the lumen as a trimer. ${ }^{418}$ The structure of the PCP complex of Amphidinium carterae, solved 20 years ago, ${ }^{417}$ showed that each monomer folds into two domains, each one containing four peridinin carotenoids and a single $C h l a$, as illustrated in Figure 9. The presence of peridinin allows dynoflagellates to absorb light in the 470-550 nm region, where chlorophylls cannot harvest light. Over the years, several studies have tried to rationalize the light-harvesting properties of PCP. Carbonera and co-workers provided a first structure-based characterization of the excitonic Hamiltonian by calculating electronic couplings from the transition monopole approximation and then fitting the site energies to spectral properties. ${ }^{421}$ The electronic interactions among peridinin and Chl $a$ were studied by Damjanovic and co-workers 
accounting both for Coulomb and exchange terms based on the semiempirical PariserParr-Pople self-consistent-field configuration interaction (PPP-SCF-CI) method. ${ }^{34,357}$ The results showed that the $\mathrm{S}_{2}$ excitations of peridinins contribute a delocalized exciton, which is however weakly coupled to the chlorophyll $\mathrm{Q}_{\mathrm{x}}$ excitation, concluding that the major energy transfer pathway $\mathrm{S}_{1} \rightarrow \mathrm{Q}_{\mathrm{y}}$ occurs after fast internal conversion to the $\mathrm{S}_{1}$ state of peridinin. Recent estimates of electronic couplings estimated using the TDC method from transition densities derived from semiempirical configuration interaction calculations, in addition, suggest that the $\mathrm{S}_{1} \rightarrow \mathrm{Q}_{\mathrm{y}}$ major energy transfer pathway may be complemented by a nonnegligible $\mathrm{S}_{2} \rightarrow \mathrm{Q}_{\mathrm{y}}$ transfer. ${ }^{419,420}$ Another study based on semiempirical calculations indicated that the charged residues within $8 \AA$ from the pigments control the spectroscopic properties in the complex. ${ }^{422}$ The study by Damjanovic et al. also suggested that triplet energy transfer to peridinins mediated by electron exchange efficiently protects the chlorophylls against photo-oxidation, ${ }^{34,357}$ and it has been proposed that a water molecule mediates the triplet energy transfer among chlorophyll and peridinin. ${ }^{176,423}$ Accurate estimates of the electronic couplings among triplet states have been predicted using the recently proposed fragment spin difference scheme, supporting the general photoprotective role of carotenoids in photosynthesis. ${ }^{172}$ In contrast to other carotenoids, it is worth noting that the presence of polar groups in peridinin has been shown to lead to excitations with a remarkable charge-transfer (CT) character, ${ }^{424}$ and early semiempirical calculations suggested the $\mathrm{S}_{1}$ state involved in the $\mathrm{S}_{1} \rightarrow \mathrm{Q}_{\mathrm{y}}$ major light-harvesting pathway in PCP had a considerable CT nature. ${ }^{425}$ Later calculations based on the TDA formulation of TDDFT, in contrast, indicated that the $\mathrm{S}_{1}$ state energy is relatively insensitive to environment polarity, whereas an additional nearby distinct CT state was identified that could explain the short lifetime of peridinin in polar solvents. ${ }^{426} \mathrm{~A}$ more recent study addressed the nature of the CT state by using semiempirical, and correlated SAC-CI and EOM-CCSD calculations, and concluded that such state arises via mixing of the $\mathrm{S}_{2}$ and $\mathrm{S}_{1}$ states. ${ }^{427}$

\subsubsection{LHC family}

The light-harvesting complexes (LHC) family includes different membrane-intrinsic chlorophyll-binding proteins present in algae and plants. Such complexes share a similar structure based on three transmembrane $\alpha$-helix regions and several highly conserved Chl-binding residues. The major antennas of this family are named LHCI and LHCII, depending on whether they are associated to the photosystem I (PSI) or photosystem II (PSII) reaction centers. The LHCI complex involves four subunits, arranged as a heterodimer of two heterodimers creating a half-moon-shaped belt on one side of the PS1 core. A recent crystal structure of the PSI-LHC1 supercomplex of Pisum sativum (pea) solved at $2.8 \AA$ resolution has revealed the detailed position and number of pigments associated to LHCI, including $45 \mathrm{Chl} a, 12 \mathrm{Chl} \mathrm{b}$ and 13 carotenoids. ${ }^{428} \mathrm{On}$ the other hand, the LHCII complex is the most abundant membrane protein in the biosphere, and is organized as a trimer, each one containing $8 \mathrm{Chl} a, 6 \mathrm{Chl} b$ and three 
to four carotenoids (see Figure 10). ${ }^{429,430}$ In addition to LHCII, the antenna apparatus of PSII contains the minor peripheral antenna complexes CP24, CP26 and CP29, ${ }^{431}$ and the core antennas CP43 and CP47 bound to PSII. The three-dimensional structures of LHCII, ${ }^{429,430} \mathrm{CP} 29^{432}$ and PSII, the latter including the CP43 and CP47 core antennae, ${ }^{433}$ have been solved by X-ray crystallography, allowing a detailed examination of their spectral and energy transfer properties in terms of the underlying pigment composition. Beyond their light-harvesting antenna function, these complexes are believed to play a key role in the nonphotochemical quenching (NPQ) mechanism that allows the dissipation of the excess energy absorbed in high light conditions as heat. $^{434}$

a)

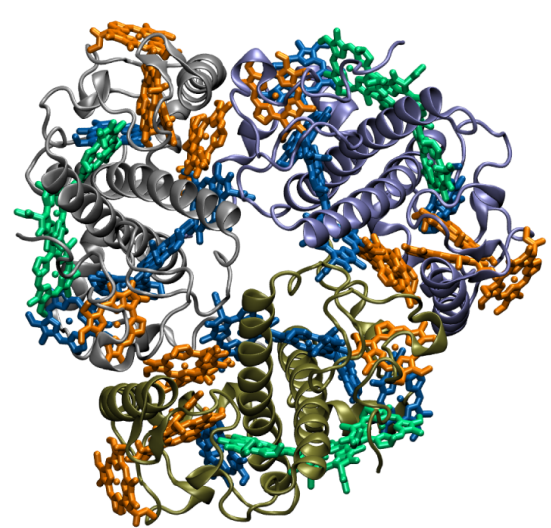

b)

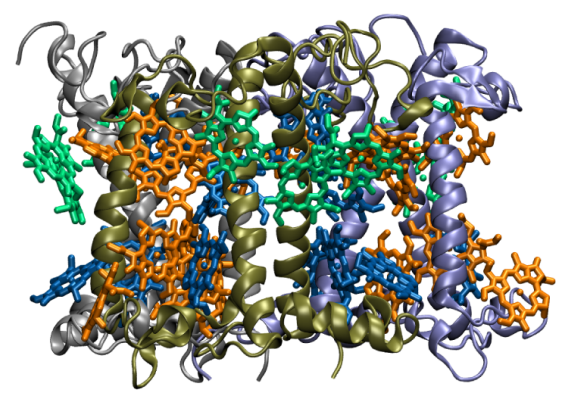

c)

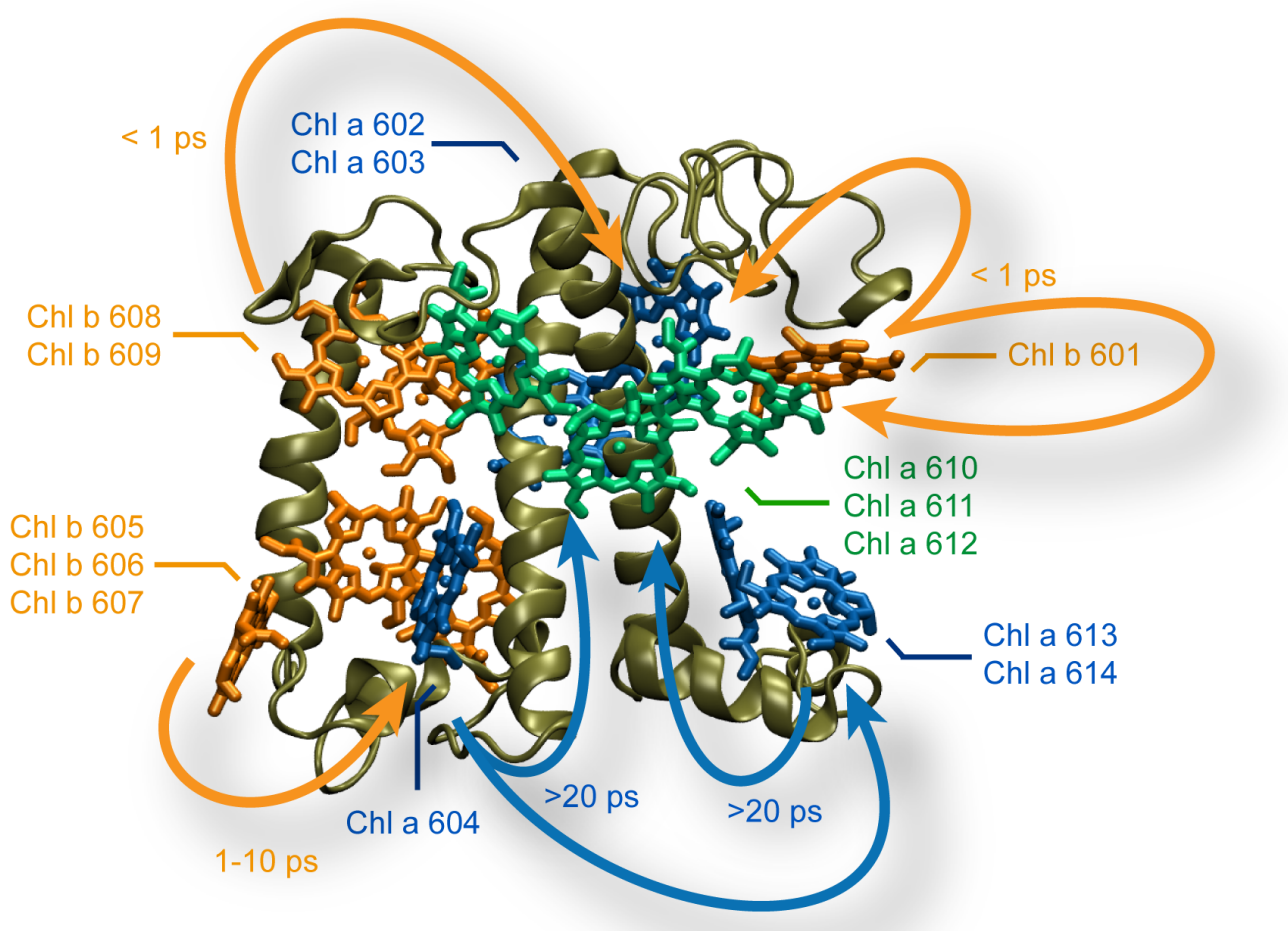

Figure 10. Structure of the LHCII major light-harvesting complex of pea ${ }^{430}$ (Chl a molecules contributing to the final energy acceptor state in green, other $C h l a$ in blue, $C h l b$ in orange). a) Top view from the stromal side. b) Side view. c) Schematic picture of energy transfer pathways in a single monomer of LHC-II indicating the approximate time scales for energy transfer. ${ }^{435}$ 
In LHC complexes, the spectral properties are determined by the degree of delocalization among chlorophylls arising from excitonic coupling, but also from the tuning exerted by pigment-protein interactions as well as the different intrinsic properties of $C h l a, C h l b$ and the contained carotenoids. Thus, both accurate estimates of electronic couplings and site energies are needed in order to rationalize their properties. In terms of estimated couplings, it was early realized that in such densely packed aggregates, the point dipole approximation could severely break down. Application of the TDC method to the LHCII complex indeed showed that the point dipole approximation can introduce important deviations at interpigment distances $<25$ $\AA .{ }^{436}$ On the other hand, several groups have attempted the determination of the site energies in LHCII based on its structure, studies often supported by the availability of spectral information for mutants that provided hints on the spectral position of some chlorophylls. ${ }^{437-442}$ Such studies included semiempirical ZINDO calculations including the effect of nearby amino acids, ${ }^{437}$ electrostatic shift calculations including the effect of the protein amino acids, ${ }^{438,440,441}$ subsystem TD-DFT calculations based on a full DFT description of the complex, ${ }^{439}$ and an analysis based on the chlorophyll macrocycle deformations observed in the crystal structure. ${ }^{442}$ However, the assignment of the site energies in the complex still remains controversial. Dreuw and co-workers, for example, have argued that geometry re-optimizations of the pigments in the crystal are needed in order to obtain reliable QM estimates not only of the site energies, but also for the correct description of the coupling among excited states. ${ }^{443}$ Analysis of the mixing of the complex excited states obtained from subsystem TD-DFT calculations further suggest that some pigments can act as bridges mediating the electronic couplings between other pigments. ${ }^{439}$ The electrostatic shift model (see section 2.2.2) so far has provided the best description of the wild type and the different mutant spectral properties, ${ }^{438,440}$ suggesting that the energy sink at cryogenic temperatures is located on Chl $a \quad 610$ in the stromal layer of pigments, although the authors note that thermal effects could modify the nature of the terminal emitter domain (Chl a 610/611/612). Encouragingly, different studies seem to agree in the location of the energy sink in this domain, including site energies derived from 2D spectroscopy, ${ }^{444}$ empirical fitting strategies, ${ }^{445}$ and structure-based calculations based on the impact of pigment-protein interactions ${ }^{438}$ or chlorophyll macrocycle deformations. ${ }^{442}$ In addition, the spectral changes between trimers and small lamellar aggregates have also been characterized from electrostatic shift calculations, suggesting that the energy sink remains the same in both states. ${ }^{441}$

Structure-based calculations either based on electrostatic shift calculations or MDQM/MM schemes have also been presented for the minor CP29138,446 and the core CP43 antenna complexes, suggesting for example that the energy sink in CP29 is different to that in LHCII due to the mutation of an amino acid, ${ }^{446}$ or illustrating the impact that the heterogeneous polarizable environment of the protein has on the interpigment couplings. ${ }^{138}$ On the other hand, a first attempt to estimate the site energies in the CP43 
complex from semiempirical ZINDO calculations where considered unreliable, given that different values were found for the two monomers of CP43 in the PSII dimer. ${ }^{447}$ By adopting electronic couplings computed using the TrESP method, Raszewski and Renger successfully derived a set of site energies both for the CP43 and CP47 core antennae of PSII. ${ }^{448}$ Later, the site energies of CP43 were refined from structure-based calculations based on electrostatic shift calculations, suggesting different mutations of potential interest that could shed further light in the assignment of the excited states in the complex. ${ }^{449,450}$

Several quantum chemical studies have also addressed the role of the xanthophyll carotenoids in the light harvesting capability of LHCII, ${ }^{451-453}$ indicating for example that carotenoids can provide a shortcut for the chlorophylls Soret-to-Q bands energy flow. ${ }^{453}$ Moreover, the role of the xantophylls is believed to be central to the ability of LHCII to dissipate the excess energy through NPQ. Several quantum chemical studies have investigated the molecular basis of the predominant NPQ component, the rapidly reversible energy quenching triggered by the $\mathrm{pH}$ difference across the thylakoid membrane. It is known that this mechanism is controlled by the PsbS protein, as well as the interconversion of different carotenoids through the xantophyll cycle, ${ }^{434}$ but the molecular basis of the process is still a subject of intense debate. Several mechanisms have been proposed, such as the generation of a zeaxanthin-to-chlorophyll chargetransfer state proposed from QM calculations ${ }^{454-460}$ and detected later spectroscopically in thylakoid membranes, ${ }^{461}$ energy transfer to the $S_{1}$ dark state of luthein ${ }^{462}$ or zeaxanthin, ${ }^{455}$ excited state mixing between $C h l$ and carotenoid, ${ }^{463}$ or the formation of dark $C h l$ dimers arranged as an $\mathrm{H}$-aggregate, ${ }^{464}$ but the issue still remains unsolved.

\subsubsection{Reaction centers}

The reaction centers (RC) perform the key charge separation events that allow converting the absorbed sunlight into a form that an organism can use to sustain and power its life processes. To avoid charge recombination, such charge transfer reactions need to be ultrafast. In contrast to antenna complexes, which have evolved into a variety of structures depending on the available sunlight in different habitats, reaction centers are highly conserved. The first anoxygenic photosynthetic organisms use a single type of RC, type II in purple bacteria and type I in green sulfur bacteria, as well as different electron donors, whereas oxygenic bacteria, algae and plants use a more sophisticated machinery composed of both type I and type II RCs, called photosystems I (PSI) and II (PSII). ${ }^{7}$ The two types of RCs differ in their ability to reduce either quinones (type II) or FeS centers (type I) as terminal acceptors. Despite significant differences in their

protein sequence, the X-ray crystal structures of both types of RCs clearly show a common evolutionary origin, where the pigments are organized in two branches related by a pseudo- $\mathrm{C}_{2}$ symmetry. 
The purple bacterial reaction centers (pBRC) contain four bacteriochlorophylls $(B C h l)$, two bacteriopheophytin $(B P h)$, one metal ion (in most cases $\mathrm{Fe}^{2+}$ ), two quinones and, in most cases, one carotenoid. ${ }^{465}$ The PSII complex has a somewhat different pigment composition, involving six chlorophyll a, two pheophytin a and two $\beta$-carotenes, where two of the chlorophylls are bound near the periphery of the complex. In its oxidized form, PSII is able to oxidize water and generate $\mathrm{O}_{2}$, and serves as inspiration for the development of artificial photosynthetic devices. ${ }^{466}$ In contrast to pBRC and PSII, the central pigments in the pseudo-C2 symmetric branches of PSI are surrounded by a collection of additional pigments, approximately 100 chlorophylls and 12-14 $\beta$ carotenes. All RCs, nevertheless, are characterized by a strongly coupled chlorophyll dimer called the special pair, which serves as the primary electron donor, as illustrated in Figure 11. The charge-transfer reactivity of RCs is out of the scope of the present paper. Here, we rather focus on the quantum chemical characterization of the excitedstates and the energy transfer processes that allow excitation of the special pair.

In contrast to PSI and PSII, in the pBRC the spectral bands arising from the special pair, the accessory BChls and the $B P h s$ are well resolved. ${ }^{467}$ This bands show a strong redshifted absorption due to the special pair BChls, and before the crystal structure of the bRC of purple bacterium Rhodopseudomonas viridis was solved in $1984,{ }^{468,469}$ quantum chemical studies first addressed the origin of such red-shifted band from semiempirical calculations performed on $B C h l$ dimers, suggesting an interpigment distance of less than $4 \AA .{ }^{470}$ Once the crystal structure was solved, several groups attempted the characterization of the pBRC spectral properties from QM calculations. Hanson and coworkers, for example, rationalized the spectral changes observed in the picosecond time scale following excitation of the bBRC by predicting the effects that the charge separation could have on spectral properties of the special pair estimated using the ZINDO method. ${ }^{471}$ Semiempirical calculations indicated that the special pair excitations could not be described using an exciton model, ${ }^{472}$ and Parson and Warshel showed that CT transitions among the BChls of the special pair significantly impact the lowest energy absorption band. ${ }^{473,474}$ Indeed, a later study based on an exciton Hamiltonian where electronic couplings were computed based on the transition monopole approximation indicated that the large splitting among the excitons of the special pair could not be explained only by exciton coupling, so that CT states or a large protein solvatochromic shift on site energies should also contribute. ${ }^{475}$ Later ZINDO calculations confirmed the similar impact of excitonic and charge transfer interactions on the red shift. ${ }^{476}$ An extension of that study to the complete pBRC including the dielectric effects exerted by the protein environment through a continuum solvation model showed the importance of the pigment-protein interactions in stabilizing CT states. Interestingly, the calculations showed that the CT states in the photoactive L branch are lower than those in the $\mathrm{M}$ branch. However, the energy of the lowest CT state, where an electron is transferred from the special pair to the $B P h$ in the $\mathrm{L}$ branch, was predicted to be $1200 \mathrm{~cm}^{-1}$ above the lowest exciton in the special pair, so it was 
proposed that structural relaxation effects could lower further its energy. ${ }^{477} \mathrm{~A}$ subsequent study explicitly incorporated the effect of five adjacent amino acids described at the QM level as well as the impact of the reminder of the protein using a polarizable MM description in the ZINDO calculation of the special pair excited states. The results showed that while formation of the $B C h l$ dimer is enough to capture the redshifted band, protein polarization was needed in order to capture the charge transfer asymmetry in the excitations observed from the experimental Stark effect. ${ }^{478} \mathrm{Hu}$ et al. also studied theoretically the energy transfer from the LH1 antenna to the pBRC, suggesting that the accessory BChls in the reaction center are critical for the LH1 $\rightarrow$ pBRC transfer. ${ }^{346,479,480}$ Hasegawa and co-workers, on the other hand, presented a detailed assignment of the spectral properties of the pBRC from correlated SAC-CI calculations on the pigments accounting for the effects of the environment through point charges. ${ }^{481-483}$ Later, fragment molecular orbital CIS calculations of the pBRC excitedstates using a full QM description of the protein environment indicated an asymmetry due to pigment-protein interactions stabilizing the excitations on the $\mathrm{L}$ branch. ${ }^{90}$ More recent semiempirical ZINDO calculations applied to the wild type and a mutant pBRC of Rhodobacter sphaeroides suggested that the lowest CT state in the complex involves not only the central pair, but also the accesory $B C h l$ of the photosynthetically active electron transfer branch. ${ }^{362}$

The observation of long-lived quantum coherence effects in the pBRC from twodimensional spectroscopy, ${ }^{184}$ initially suggested to arise from correlated fluctuations on the pigments excited states induced by the protein environment, lead to a detailed study on the pigment-protein coupling of the $\mathrm{Q}_{\mathrm{y}}$ states in the pBRC by combining MD simulations with ZINDO and electrostatic shift calculations. ${ }^{256}$ In that study, the intramolecular contribution to the spectral density of electronic-vibrational coupling was quite accurately obtained from a QM normal mode analysis, which showed that the "geometry mismatch" problem previously observed in calculations combining classical MD simulations and QM calculations can lead to a strong overestimation of the electronic-vibrational coupling.

In contrast to the $\mathrm{pBRC}$, the PSII complex displays a highly-congested spectrum that greatly complicates the characterization of its underlying excited-states. ${ }^{484}$ Several groups have postulated a multimer model describing the lowest excited-state in the complex as an exciton delocalized over several pigments, both due to a smaller coupling in the special pair and an increased interaction with the other pigments compared to the pBRC. ${ }^{485-488}$ The increased coupling in the special pair of pBRC compared to PSII was initially thought to arise from a smaller interpigment distance. However, the analysis of the structures of PSII and pBRC indicates that the main difference arises from a tilt rather than from an increased separation. Detailed analyses of the Coulomb and shortrange contributions to the interactions of these special pairs indicated that the increased interaction in the pBRC arises from short-range contributions to the coupling, as 
illustrated in Figure 11. ${ }^{489}$ The determination of the site energies in PSII remains still controversial, and has been attempted from both empirical spectral fittings ${ }^{486,487,490}$ and more recently from structure-based calculations based on TD-DFT calculations performed on DFT-optimized geometries, ${ }^{491} \mathrm{QM} / \mathrm{MM}$ or electrostatic shift calculations performed along an MD simulation in order to account for protein dynamics ${ }^{249,492}$ and CIS supermolecule calculations on the complete chlorophyll hexamer. ${ }^{493}$ Recently, the crystal structure of the PSII complex of Thermosynechococcus vulcanus has been refined at $1.9 \AA$ resolution, allowing determination of the positions of the atoms involved in the $\mathrm{Mn}_{4} \mathrm{CaO}_{5}$ cluster of the oxygen-evolving complex. ${ }^{433}$ The new structure, in addition, suggests some structural differences among the active and inactive chains, and has been used in more recent structure-based studies by Zhang et al. in order to explore the impact of protein dynamics, explored using MD simulations, on semiempirical QM/MM estimates of the site energies, ${ }^{494}$ and by Frankcombe to compute the excited-states in the complex from a multi-monomer supermolecule calculation performed at the TD-DFT level using the long-range corrected CAMB3LYP functional. ${ }^{495}$ Interestingly, the study by Zhang et al. suggests that protein dynamics preferentially drive the energy transfer along the active chain of PSII.

a)

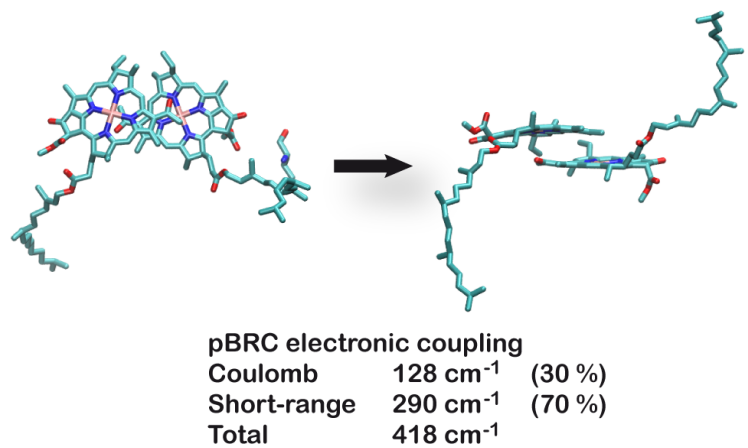

b)

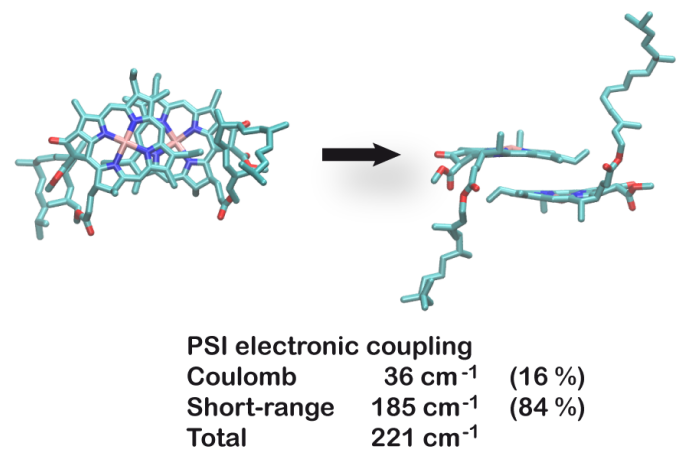

Figure 11. Estimated Coulomb and short-range contributions to the electronic couplings between $\mathrm{Q}_{\mathrm{y}}$ excitations of the chlorophylls in the special pair. ${ }^{489}$ a) $\mathrm{RC}$ of the purple bacterium Rhodobacter sphaeroides. ${ }^{496}$ b) PSI complex of the cyanobacterium Thermosynechococcus elongatus. ${ }^{497}$

The large collection of pigments that surround the pseudo-symmetric branches of pigments in PSI further complicates the characterization of the many excited-states in the complex compared to other RCs. Damjanovic first attempted the characterization of 
the PSI excited-states by performing ZINDO calculations on the 96 chlorophylls in the photosystem I of Synechococcus elongatus, allowing a tentative assignment of the red chlorophylls in the absorption spectrum. ${ }^{498}$ The energy transfer kinetics in PSI were also modeled from electronic couplings derived from the point dipole approximation ${ }^{499}$ or from QM calculations. ${ }^{500,501}$ Later, the calculation of site energies in PSI has been attempted by using DFT and semiempirical calculations including environment effects and long- and short-range contributions to electronic couplings, ${ }^{502}$ suggesting the special pair in PSI appears to be a dimer of dimers, or from electrostatic shift calculations, which suggest a light-harvesting asymmetry arising from the concentration of low energy exciton states in the A-branch. ${ }^{503}$ A further study assessed the role of dielectric screening effects in the electronic couplings in PSI from continuum dielectric calculations based on the Poisson-TrESP method (see section 3.1.2.1) ${ }^{504}$ The results indicated that, in contrast to what found in other photosynthetic complexes, ${ }^{133,134}$ screening effects are not distance-dependent in PSI, but rather depend on the mutual orientation of the pigments, an effect also predicted by PCM calculations. ${ }^{134}$ Finally, it is worth noting that a recent crystal structure of the PSI-LHC1 supercomplex of Pisum sativum (pea) has been solved at $2.8 \AA$ resolution, revealing the detailed arrangement of the pigments in LHCI. This new structure provides a firm structural basis for investigating the energy transfer and photoprotection mechanisms within the PSI-LHCI supercomplex. ${ }^{428}$

\section{CONCLUSIONS AND OUTLOOK}

Light-harvesting processes in natural photosynthetic systems have attracted the interest of experimentalists and theoreticians since many years; it is sufficient to recall here the pioneering studies of Giacomo Ciamician who, in 1912, wrote in Science that "the photochemical processes that hitherto have been the guarded secret of the plants ... will have been mastered by human industry". ${ }^{505}$

More than a century has passed and the sunlight-initiated cascade of steps ultimately producing the charge separation in the reaction centers is now deeply understood. Experimental and theoretical studies have in fact revealed not only the general physical processes but also the detailed molecular mechanisms beyond them. This knowledge however has not yet meant that we can reproduce the same processes with the same characteristics of efficiency, robustness and adaptability in artificial systems. The main difficulty is that many time and length scales are involved and quantum and classical behaviors seem to be present at the same time.

In the last years, the strategy which has shown to be the most effective has been "topdown": experimental observations (mostly of spectroscopic nature) have been used to build up model Hamiltonians from which the quantum dynamics has been derived. Thanks to this strategy, many important aspects of the light-harvesting process have been captured, such as coherence effects and the role of the competition between 
dissipative and free evolution, but the real key elements of the light-harvesting function have still to be exhaustively explained. The reason for this difficulty is that all the advanced theories developed so far are necessarily limited to model systems, thus missing the unique chemical and structural complexity which characterizes the PPCs and which seems to be the main element for a successful realization of their function. A possible new way to look at the problem is "bottom-up" instead that "top-down" and it passes through the use of quantum chemical approaches. As we have shown in this review, these approaches start from the atomistic nature of the systems and from there they build up the model using multiscale tools which allow to selectively switch on the actors into play, the interacting pigments, the protein matrix, the external environment. The strongest point of these methods is however also their main limit. The atomistic nature of the model and the consideration of the many interactions among all the components have so far limited their applications mostly to the simulation of spectroscopic properties ${ }^{21,506}$ due to the unfeasible computational cost that a timedependent simulation of the full system would imply. This limitation is however not inherent in the method but instead strongly connected to the available computational algorithms and facilities. ${ }^{507}$ Some examples showing the potentials of the method have been already presented by combining the unique computational performances of GPUs with highly parallelized computational codes to perform ab initio nonadiabatic dynamics of multichromophore complexes. ${ }^{508}$ We are confident that in the next ten years quantum chemistry combined with accurate embedding models either of classical or quantum or, more probably, of hybrid nature, will allow not only to unveil the real role that the biological complexity has on the light-harvesting function in natural systems but also to design, "virtually" test and ultimately suggest the synthesis of more efficient and robust artificial light-harvesting systems.

\section{Biographies}

Carles Curutchet received his Ph.D. in Theoretical and Computational Chemistry at the University of Barcelona in 2005. After spending several years as a postdoctoral researcher in Pisa (2005), Parma (2006-2007), Toronto (2008-2009) and Girona (20102011), he was awarded a Ramón y Cajal position and joined the University of Barcelona. His research focuses on the theoretical study of resonance electronic energy transfer processes in biosystems, with particular attention to the role played by the molecular environment.

Benedetta Mennucci completed her Ph.D. in Theoretical and Computational Chemistry at the University of Pisa in 1999. Since 2012 she is full professor of theoretical chemistry at the University of Pisa. Her main research interests are the development of polarizable QM/classical methods to describe properties and processes of 
(supra)molecular systems and their applications to problems in chemistry, biology, and materials science.

\section{Acknowledgements}

C. C. acknowledges support from the Ministerio de Economía y Competitividad (MINECO) of Spain (grants CTQ2012-36195 and RYC2011-08918) and the Agència de Gestió d'Ajuts Universitaris i de Recerca from Generalitat de Catalunya (GENCAT) (SGR2014-1189). B. M. acknowledges the European Research Council (ERC) through the Starting Grant proposal n. 277755 (EnLight). 


\section{References}

(1) Fenna, R. E.; Matthews, B. W. Chlorophyll Arrangement in a Bacteriochlorophyll Protein from Chlorobium Limicola. Nature 1975, 258, 573577.

(2) Pullerits, T.; Sundström, V. Photosynthetic Light-Harvesting Pigment-Protein Complexes: Toward Understanding How and Why. Acc. Chem. Res. 1996, 29, 381-389.

(3) Ritz, T.; Damjanovic, A.; Schulten, K. The Quantum Physics of Photosynthesis. Chemphyschem 2002, 3, 243-248.

(4) Scholes, G. D. Long-Range Resonance Energy Transfer in Molecular Systems. Annu. Rev. Phys. Chem. 2003, 54, 57-87.

(5) Cheng, Y. C.; Fleming, G. R. Dynamics of Light Harvesting in Photosynthesis. Annu. Rev. Phys. Chem. 2009, 60, 241-262.

(6) Renger, T. Theory of Excitation Energy Transfer: From Structure to Function. Photosynth. Res. 2009, 102, 471-485.

(7) Collini, E.; Curutchet, C.; Mirkovic, T.; Scholes, G. D. Electronic Energy Transfer in Photosynthetic Antenna Systems. In Energy Transfer Dynamics in Biomaterial Systems; Burghardt, I., May, V., Micha, D. A., Bittner, E. R., Eds.; Springer: Berlin, 2009; pp 3-34.

(8) Beljonne, D.; Curutchet, C.; Scholes, G. D.; Silbey, R. J. Beyond Förster Resonance Energy Transfer in Biological and Nanoscale Systems. J. Phys. Chem. B 2009, 113, 6583-6599.

(9) Novoderezhkin, V. I.; van Grondelle, R. Physical Origins and Models of Energy Transfer in Photosynthetic Light-Harvesting. Phys. Chem. Chem. Phys. 2010, 12, 7352-7365.

(10) Ishizaki, A.; Calhoun, T. R.; Schlau-Cohen, G. S.; Fleming, G. R. Quantum Coherence and Its Interplay with Protein Environments in Photosynthetic Electronic Energy Transfer. Phys. Chem. Chem. Phys. 2010, 12, 7319-7337.

(11) Scholes, G. D.; Fleming, G. R.; Olaya-Castro, A.; van Grondelle, R. Lessons from Nature about Solar Light Harvesting. Nat. Chem. 2011, 3, 763-774.

(12) Ruban, A. V; Johnson, M. P.; Duffy, C. D. P. Natural Light Harvesting: Principles and Environmental Trends. Energy Environ. Sci. 2011, 4, 1643.

(13) Olaya-Castro, A.; Scholes, G. D. Energy Transfer from Forster-Dexter Theory to Quantum Coherent Light-Harvesting. Int. Rev. Phys. Chem. 2011, 30, 49-77.

(14) Fleming, G. R.; Schlau-Cohen, G. S.; Amarnath, K.; Zaks, J. Design Principles of Photosynthetic Light-Harvesting. Faraday Discuss. 2012, 155, $27-41$.

(15) Scholes, G. D.; Mirkovic, T.; Turner, D. B.; Fassioli, F.; Buchleitner, A. Solar Light Harvesting by Energy Transfer: From Ecology to Coherence. Energy Environ. Sci. 2012, 5, 9320-9374.

(16) Pachón, L. A.; Brumer, P. Computational Methodologies and Physical Insights into Electronic Energy Transfer in Photosynthetic Light-Harvesting Complexes. Phys. Chem. Chem. Phys. 2012, 14, 10094-10108.

(17) Renger, T.; Müh, F. Understanding Photosynthetic Light-Harvesting: A Bottom up Theoretical Approach. Phys. Chem. Chem. Phys. 2013, 15, 3348-3371.

(18) Anna, J. M.; Scholes, G. D.; van Grondelle, R. A Little Coherence in Photosynthetic Light Harvesting. Bioscience 2014, 64, 14-25.

(19) Croce, R.; van Amerongen, H. Natural Strategies for Photosynthetic Light Harvesting. Nat. Chem. Biol. 2014, 10, 492-501.

(20) Chenu, A.; Scholes, G. D. Coherence in Energy Transfer and Photosynthesis. 
Annu. Rev. Phys. Chem. 2015, 66, 69-96.

(21) König, C.; Neugebauer, J. Quantum Chemical Description of Absorption Properties and Excited-State Processes in Photosynthetic Systems.

ChemPhysChem 2012, 13, 386-425.

(22) Barbatti, M. Nonadiabatic Dynamics with Trajectory Surface Hopping Method. Wiley Interdiscip. Rev. Comput. Mol. Sci. 2011, 1, 620-633.

(23) Tully, J. C. Perspective: Nonadiabatic Dynamics Theory. J. Chem. Phys. 2012, 137, 22A301.

(24) Persico, M.; Granucci, G. An Overview of Nonadiabatic Dynamics Simulations Methods, with Focus on the Direct Approach versus the Fitting of Potential Energy Surfaces. Theor. Chem. Acc. 2014, 133, 1526.

(25) Tavernelli, I. Nonadiabatic Molecular Dynamics Simulations: Synergies between Theory and Experiments. Acc. Chem. Res. 2015, 48, 792-800.

(26) Subotnik, J. E.; Alguire, E. C.; Ou, Q.; Landry, B. R.; Fatehi, S. The Requisite Electronic Structure Theory To Describe Photoexcited Nonadiabatic Dynamics: Nonadiabatic Derivative Couplings and Diabatic Electronic Couplings. Acc. Chem. Res. 2015, 48, 1340-1350.

(27) Ishizaki, A.; Fleming, G. R. Quantum Coherence in Photosynthetic Light Harvesting. Annu. Rev. Condens. Matter Phys. 2012, 3, 333-361.

(28) Jang, S.; Cheng, Y. C. Resonance Energy Flow Dynamics of Coherently Delocalized Excitons in Biological and Macromolecular Systems: Recent Theoretical Advances and Open Issues. Wiley Interdiscip. Rev. Comput. Mol. Sci. 2013, 3, 84-104.

(29) Plenio, M. B.; Huelga, S. F. Dephasing-Assisted Transport: Quantum Networks and Biomolecules. New J. Phys. 2008, 10, 113019.

(30) Rebentrost, P.; Mohseni, M.; Kassal, I.; Lloyd, S.; Aspuru-Guzik, A. Environment-Assisted Quantum Transport. New J. Phys. 2009, 11, 033003.

(31) Ishizaki, A.; Fleming, G. R. Unified Treatment of Quantum Coherent and Incoherent Hopping Dynamics in Electronic Energy Transfer: Reduced Hierarchy Equation Approach. J. Chem. Phys. 2009, 130, 234111.

(32) Shi, Q.; Chen, L.; Nan, G.; Xu, R. X.; Yan, Y. Efficient Hierarchical Liouville Space Propagator to Quantum Dissipative Dynamics. J. Chem. Phys. 2009, 130, 084105.

(33) Damjanovic, A.; Ritz, T.; Schulten, K. Energy Transfer between Carotenoids and Bacteriochlorophylls in Light-Harvesting Complex II of Purple Bacteria. Phys. Rev. E 1999, 59, 3293-3311.

(34) Damjanovic, A.; Ritz, T.; Schulten, K. Excitation Transfer in the PeridininChlorophyll-Protein of Amphidinium Carterae. Biophys. J. 2000, 79, 1695-1705.

(35) Ridley, J.; Zerner, M. An Intermediate Neglect of Differential Overlap Technique for Spectroscopy: Pyrrole and the Azines. Theor. Chim. Acta 1973, 32, 111-134.

(36) Liu, X.; Subotnik, J. E. The Variationally Orbital-Adapted Configuration Interaction Singles (VOA-CIS) Approach to Electronically Excited States. $J$. Chem. Theory Comput. 2014, 10, 1004-1020.

(37) Veldkamp, B. S.; Liu, X.; Wasielewski, M. R.; Subotnik, J. E.; Ratner, M. A. Molecular Excited States: Accurate Calculation of Relative Energies and Electronic Coupling Between Charge Transfer and Non-Charge Transfer States. J. Phys. Chem. A 2015, 119, 253-262.

(38) Casida, M. E. Time-dependent Density Functional Response Theory of Molecular Systems: Theory, Computational Methods, and Functionals. In Recent Developments and Applications of Modern Density Functional Theory; 
Seminario, J. M., Ed.; Elsevier Science: Amsterdam, 1996; pp 399-402.

(39) Peach, M. J. G.; Williamson, M. J.; Tozer, D. J. Influence of Triplet Instabilities in TDDFT. J. Chem. Theory Comput. 2011, 7, 3578-3585.

(40) Chai, J. Da; Head-Gordon, M. Systematic Optimization of Long-Range Corrected Hybrid Density Functionals. J. Chem. Phys. 2008, 128, 1-15.

(41) Higashi, M.; Kosugi, T.; Hayashi, S.; Saito, S. Theoretical Study on Excited States of Bacteriochlorophyll Ain Solutions with Density Functional Assessment. J. Phys. Chem. B 2014, 118, 10906-10918.

(42) Vydrov, O. A.; Scuseria, G. E. Assessment of a Long-Range Corrected Hybrid Functional. J. Chem. Phys. 2006, 125, 1-9.

(43) Yanai, T.; Tew, D. P.; Handy, N. C. A New Hybrid Exchange-correlation Functional Using the Coulomb-Attenuating Method (CAM-B3LYP). Chem. Phys. Lett. 2004, 393, 51-57.

(44) Jacquemin, D.; Moore, B.; Planchat, A.; Adamo, C.; Autschbach, J. Performance of an Optimally Tuned Range-Separated Hybrid Functional for 0-0 Electronic Excitation Energies. J. Chem. Theory Comput. 2014, 10, 1677-1685.

(45) Kuritz, N.; Stein, T.; Baer, R.; Kronik, L. Charge-Transfer-like $\pi \rightarrow \pi^{*}$ Excitations in Time-Dependent Density Functional Theory: A Conundrum and Its Solution. J. Chem. Theory Comput. 2011, 7, 2408-2415.

(46) Bokareva, O. S.; Grell, G.; Bokarev, S. I.; Kühn, O. Tuning Range-Separated Density Functional Theory for Photocatalytic Water Splitting Systems. J. Chem. Theory Comput. 2015, 11, 1700-1709.

(47) Krykunov, M.; Seth, M.; Ziegler, T. Introducing Constricted Variational Density Functional Theory in Its Relaxed Self-Consistent Formulation (RSCF-CV-DFT) as an Alternative to Adiabatic Time Dependent Density Functional Theory for Studies of Charge Transfer Transitions. J. Chem. Phys. 2014, 140, 18A502.

(48) Kaduk, B.; Kowalczyk, T.; Van Voorhis, T. Constrained Density Functional Theory. Chem. Rev. 2012, 112, 321-370.

(49) Ratcliff, L. E.; Grisanti, L.; Genovese, L.; Deutsch, T.; Neumann, T.; Danilov, D.; Wenzel, W.; Beljonne, D.; Cornil, J. Toward Fast and Accurate Evaluation of Charge On-Site Energies and Transfer Integrals in Supramolecular Architectures Using Linear Constrained Density Functional Theory (CDFT)-Based Methods. $J$. Chem. Theory Comput. 2015, 11, 2077-2086.

(50) Solovyeva, A.; Pavanello, M.; Neugebauer, J. Describing Long-Range ChargeSeparation Processes with Subsystem Density-Functional Theory. J. Chem. Phys. 2014, 140, 164103.

(51) Holmgaard List, N.; Curutchet, C.; Knecht, S.; Mennucci, B.; Kongsted, J. Toward Reliable Prediction of the Energy Ladder in Multichromophoric Systems: A Benchmark Study on the FMO Light-Harvesting Complex. J. Chem. Theory Comput. 2013, 9, 4928-4938.

(52) Grimme, S.; Waletzke, M. A Combination of Kohn-Sham Density Functional Theory and Multi-Reference Configuration Interaction Methods. J. Chem. Phys. 1999, 111, 5645.

(53) Andreussi, O.; Knecht, S.; Marian, C. M.; Kongsted, J.; Mennucci, B. Carotenoids and Light-Harvesting: From DFT/MRCI to the Tamm-Dancoff Approximation. J. Chem. Theory Comput. 2015, 11, 655-666.

(54) Tao, J.; Perdew, J. P.; Staroverov, V. N.; Scuseria, G. E. Climbing the Density Functional Ladder: Nonempirical Meta-Generalized Gradient Approximation Designed for Molecules and Solids. Phys. Rev. Lett. 2003, 91, 146401.

Morzan, U. N.; Ramírez, F. F.; Oviedo, M. B.; Sánchez, C. G.; Scherlis, D. A.; 
Lebrero, M. C. G. Electron Dynamics in Complex Environments with Real-Time Time Dependent Density Functional Theory in a QM-MM Framework. J. Chem. Phys. 2014, 140, 164105.

(56) Tussupbayev, S.; Govind, N.; Lopata, K.; Cramer, C. J. Comparison of RealTime and Linear-Response Time-Dependent Density Functional Theories for Molecular Chromophores Ranging from Sparse to High Densities of States. J. Chem. Theory Comput. 2015, 11, 1102-1109.

(57) Yabana, K.; Bertsch, G. F. Time-Dependent Local-Density Approximation in Real Time. Phys. Rev. B 1996, 54, 4484-4487.

(58) Li, X.; Smith, S. M.; Markevitch, A. N.; Romanov, D. A.; Levis, R. J.; Schlegel, H. B. A Time-Dependent Hartree-Fock Approach for Studying the Electronic Optical Response of Molecules in Intense Fields. Phys. Chem. Chem. Phys. 2005, 7, 233-239.

(59) Castro, A.; Appel, H.; Oliveira, M.; Rozzi, C. A.; Andrade, X.; Lorenzen, F.; Marques, M. A. L.; Gross, E. K. U.; Rubio, A. Octopus: A Tool for the Application of Time-Dependent Density Functional Theory. Phys. Status Solidi Basic Res. 2006, 243, 2465-2488.

(60) Meng, S.; Kaxiras, E. Real-Time, Local Basis-Set Implementation of TimeDependent Density Functional Theory for Excited State Dynamics Simulations. J. Chem. Phys. 2008, 129, 054110.

(61) Peng, B.; Lingerfelt, D. B.; Ding, F.; Aikens, C. M.; Li, X. Real-Time TDDFT Studies of Exciton Decay and Transfer in Silver Nanowire Arrays. J. Phys. Chem. C 2015, 119, 6421-6427.

(62) Andrea Rozzi, C.; Maria Falke, S.; Spallanzani, N.; Rubio, A.; Molinari, E.; Brida, D.; Maiuri, M.; Cerullo, G.; Schramm, H.; Christoffers, J.; Lienau, C. Quantum Coherence Controls the Charge Separation in a Prototypical Artificial Light-Harvesting System. Nat. Commun. 2013, 4, 1602-1607.

(63) Caricato, M.; Curutchet, C.; Mennucci, B.; Scalmani, G. Electronic Couplings for Resonance Energy Transfer from CCSD Calculations: From Isolated to Solvated Systems. J. Chem. Theory Comput. 2015, 11, 5219-5228.

(64) Höfener, S.; Visscher, L. Wave Function Frozen-Density Embedding: Coupled Excitations. J. Chem. Theory Comput. 2016, 12, 549-557.

(65) Anda, A.; Hansen, T.; De Vico, L. Multireference Excitation Energies for Bacteriochlorophylls A within Light Harvesting System 2. J. Chem. Theory Comput. 2016, DOI: 10.1021/acs.jctc.5b01104.

(66) Mennucci, B. Polarizable Continuum Model. WIREs Comput. Mol. Sci. 2012, 2, 386-404.

(67) Cancès, E.; Mennucci, B.; Tomasi, J.; Cances, E.; Mennucci, B.; Tomasi, J. A New Integral Equation Formalism for the Polarizable Continuum Model: Theoretical Background and Applications to Isotropic and Anisotropic Dielectrics. J. Chem. Phys. 1997, 107, 3032-3041.

(68) Barone, V.; Cossi, M. Quantum Calculation of Molecular Energies and Energy Gradients in Solution by a Conductor Solvent Model. J. Phys. Chem. A 1998, 5639, 1995-2001.

(69) Chipman, D. M. Charge Penetration in Dielectric Models of Solvation. J. Chem. Phys. 1997, 106, 10194.

(70) Klamt, A. The COSMO and COSMO-RS Solvation Models. Wiley Interdiscip. Rev. Comput. Mol. Sci. 2011, 1, 699-709.

(71) Lipparini, F.; Barone, V. Polarizable Force Fields and Polarizable Continuum Model: A Fluctuating charges/PCM Approach. 1. Theory and Implementation. $J$. 
Chem. Theory Comput. 2011, 7, 3711-3724.

(72) Boulanger, E.; Thiel, W. Solvent Boundary Potentials for Hybrid QM/MM Computations Using Classical Drude Oscillators: A Fully Polarizable Model. $J$. Chem. Theory Comput. 2012, 8, 4527-4538.

(73) Cieplak, P.; Dupradeau, F.-Y. Y.; Duan, Y.; Wang, J. M. Polarization Effects in Molecular Mechanical Force Fields. J. Physics-Condensed Matter 2009, 21, 333102.

(74) Thole, B. T. Molecular Polarizabilities Calculated with a Modified Dipole Interaction. Chem. Phys. 1981, 59, 341-350.

(75) van Duijnen, P. T.; Swart, M. Molecular and Atomic Polarizabilities: Thole's Model Revisited. J. Phys. Chem. A 1998, 102, 2399-2407.

(76) Bryce, R. A.; Buesnel, R.; Hillier, I. H.; Burton, N. A. A Solvation Model Using a Hybrid Quantum Mechanical/molecular Mechanical Potential with Fluctuating Solvent Charges. Chem. Phys. Lett. 1997, 279, 367-371.

(77) Caricato, M.; Mennucci, B.; Tomasi, J.; Ingrosso, F.; Cammi, R.; Corni, S.; Scalmani, G. Formation and Relaxation of Excited States in Solution: A New Time Dependent Polarizable Continuum Model Based on Time Dependent Density Functional Theory. J. Chem. Phys. 2006, 124, 1-13.

(78) Improta, R.; Barone, V.; Scalmani, G.; Frisch, M. J. A State-Specific Polarizable Continuum Model Time Dependent Density Functional Theory Method for Excited State Calculations in Solution. J. Chem. Phys. 2006, 125, 054103.

(79) Marenich, A. V.; Cramer, C. J.; Truhlar, D. G.; Guido, C. A.; Mennucci, B.; Scalmani, G.; Frisch, M. J. Practical Computation of Electronic Excitation in Solution: Vertical Excitation Model. Chem. Sci. 2011, 2, 2143.

(80) Guido, C. A.; Jacquemin, D.; Adamo, C.; Mennucci, B. Electronic Excitations in Solution: The Interplay between State Specific Approaches and a TimeDependent Density Functional Theory Description. J. Chem. Theory Comput. 2015, 11, 5782-5790.

(81) Renger, T.; Grundkötter, B.; Madjet, M. E.-A.; Müh, F. Theory of Solvatochromic Shifts in Nonpolar Solvents Reveals a New Spectroscopic Rule. Proc. Natl. Acad. Sci. U. S. A. 2008, 105, 13235-13240.

(82) Marenich, A. V; Cramer, C. J.; Truhlar, D. G. Uniform Treatment of SoluteSolvent Dispersion in the Ground and Excited Electronic States of the Solute Based on a Solvation Model with State-Specific Polarizability. J. Chem. Theory Comput. 2013, 9, 3649-3659.

(83) Cupellini, L.; Amovilli, C.; Mennucci, B. Electronic Excitations in Nonpolar Solvents: Can the Polarizable Continuum Model Accurately Reproduce Solvent Effects? J. Phys. Chem. B 2015, 119, 8984-8991.

(84) Amovilli, C.; Mennucci, B. Self-Consistent-Field Calculation of Pauli Repulsion and Dispersion Contributions to the Solvation Free Energy in the Polarizable Continuum Model. J. Phys. Chem. B 1997, 101, 1051-1057.

(85) Muh, F.; Madjet, M. E.-A.; Adolphs, J.; Abdurahman, A.; Rabenstein, B.; Ishikita, H.; Knapp, E.-W.; Renger, T. $\alpha$-Helices Direct Excitation Energy Flow in the Fenna Matthews Olson Protein. Proc. Natl. Acad. Sci. 2007, 104, 1686216867.

(86) Adolphs, J.; Muh, F.; Madjet, M. E. A.; Renger, T. Calculation of Pigment Transition Energies in the FMO Protein. Photosynth. Res. 2008, 95, 197-209.

(87) Fedorov, D. G.; Kitaura, K. Extending the Power of Quantum Chemistry to Large Systems with the Fragment Molecular Orbital Method. J. Phys. Chem. A 2007, 111, 6904-6914. 
(88) Gao, J.; Truhlar, D. G.; Wang, Y.; Mazack, M. J. M.; Löffler, P.; Provorse, M. R.; Rehak, P. Explicit Polarization: A Quantum Mechanical Framework for Developing Next Generation Force Fields. Acc. Chem. Res. 2014, 47, 28372845.

(89) Gordon, M. S.; Freitag, M. A.; Bandyopadhyay, P.; Jensen, J. H.; Kairys, V.; Stevens, W. J. The Effective Fragment Potential Method: A QM-Based MM Approach to Modeling. J. Phys. Chem. A 2001, 105, 293-307.

(90) Ikegami, T.; Ishida, T.; Fedorov, D. G.; Kitaura, K.; Inadomi, Y.; Umeda, H.; Yokokawa, M.; Sekiguchi, S. Fragment Molecular Orbital Study of the Electronic Excitations in the Photosynthetic Reaction Center of Blastochloris Viridis. J. Comput. Chem. 2010, 31, 447-454.

(91) DeFusco, A.; Minezawa, N.; Slipchenko, L. V; Zahariev, F.; Gordon, M. S. Modeling Solvent Effects on Electronic Excited States. J. Phys. Chem. Lett. 2011, 2, 2184-2192.

(92) Cortona, P. Self-Consistently Determined Properties of Solids without BandStructure Calculations. Phys. Rev. B 1991, 44, 8454-8458.

(93) Wesolowski, T. A.; Warshel, A. Frozen Density Functional Approach for Ab Initio Calculations of Solvated Molecules. J. Phys. Chem. A 1993, 97, 80508053.

(94) Jacob, C. R.; Neugebauer, J. Subsystem Density-Functional Theory. Wiley Interdiscip. Rev. Comput. Mol. Sci. 2014, 4, 325-362.

(95) Wesolowski, T. A.; Shedge, S.; Zhou, X. Frozen-Density Embedding Strategy for Multilevel Simulations of Electronic Structure. Chem. Rev. 2015, 115, 58915928.

(96) Casida, M. E.; Wesolowski, T. A. Generalization of the Kohn-Sham Equations with Constrained Electron Density Formalism and Its Time-Dependent Response Theory Formulation. Int. J. Quantum Chem. 2004, 96, 577-588.

(97) Wesolowski, T. A. Hydrogen-Bonding-Induced Shifts of the Excitation Energies in Nucleic Acid Bases: An Interplay between Electrostatic and Electron Density Overlap Effects. J. Am. Chem. Soc. 2004, 126, 11444-11445.

(98) Neugebauer, J. Couplings between Electronic Transitions in a Subsystem Formulation of Time-Dependent Density Functional Theory. J. Chem. Phys. 2007, 126, 134116.

(99) König, C.; Neugebauer, J. Protein Effects on the Optical Spectrum of the FennaMatthews-Olson Complex from Fully Quantum Chemical Calculations. J. Chem. Theory Comput. 2013, 9, 1808-1820.

(100) Zhang, D. W.; Zhang, J. Z. H. Molecular Fractionation with Conjugate Caps for Full Quantum Mechanical Calculation of Protein-molecule Interaction Energy. $J$. Chem. Phys. 2003, 119, 3599.

(101) Jacob, C. R.; Visscher, L. A Subsystem Density-Functional Theory Approach for the Quantum Chemical Treatment of Proteins. J. Chem. Phys. 2008, 128, 155102.

(102) Kiewisch, K.; Jacob, C. R.; Visscher, L. Quantum-Chemical Electron Densities of Proteins and of Selected Protein Sites from Subsystem Density Functional Theory. J. Chem. Theory Comput. 2013, 9, 2425-2440.

(103) Wesolowski, T. A.; Weber, J. Kohn-Sham Equations with Constrained Electron Density: An Iterative Evaluation of the Ground-State Electron Density of Interacting Molecules. Chem. Phys. Lett. 1996, 248, 71-76.

(104) Goez, A.; Jacob, C. R.; Neugebauer, J. Modeling Environment Effects on Pigment Site Energies: Frozen Density Embedding with Fully QuantumChemical Protein Densities. Comput. Theor. Chem. 2014, 1040-1041, 347-359. 
(105) Dexter, D. L. A Theory of Sensitized Luminescence in Solids. J. Chem. Phys. 1953, 21, 836-850.

(106) You, Z. Q.; Hsu, C. P.; Fleming, G. R. Triplet-Triplet Energy-Transfer Coupling: Theory and Calculation. J. Chem. Phys. 2006, 124, 44506.

(107) Ohta, K.; Closs, G. L.; Morokuma, K.; Green, N. J. Stereoelectronic Effects in Intramolecular Long-Distance Electron Transfer in Radical Anions as Predicted by Ab-Initio MO Calculations. J. Am. Chem. Soc. 1986, 108, 1319-1320.

(108) Farazdel, A.; Dupuis, M.; Clementi, E.; Aviram, A. Electric-Field Induced Intramolecular Electron Transfer in Spiro .pi.-Electron Systems and Their Suitability as Molecular Electronic Devices. A Theoretical Study. J. Am. Chem. Soc. 1990, 112, 4206-4214.

(109) Yeganeh, S.; Van Voorhis, T. Triplet Excitation Energy Transfer with Constrained Density Functional Theory. J. Phys. Chem. C 2010, 114, 2075620763.

(110) Yost, S. R.; Hontz, E.; Yeganeh, S.; Van Voorhis, T. Triplet vs Singlet Energy Transfer in Organic Semiconductors: The Tortoise and the Hare. J. Phys. Chem. C 2012, 116, 17369-17377.

(111) Si, Y.; Liang, W.; Zhao, Y. Theoretical Prediction of Triplet-Triplet Energy Transfer Rates in a Benzophenone-Fluorene-Naphthalene System. J. Phys. Chem. C 2012, 116, 12499-12507.

(112) Si, Y.; Yang, B.; Qin, H.; Yuan, J.; Wang, S.; Chen, H.; Zhao, Y. Atomistic Modeling of Triplet-Triplet Energy-Transfer Rates from Drug (S)-Propranolol to (R)-Cinacalcet in Human a1-Acid Glycoprotein. J. Phys. Chem. C 2015, 119, 8014-8022.

(113) Muñoz-Losa, A.; Curutchet, C.; Krueger, B. P.; Hartsell, L. R.; Mennucci, B. Fretting about FRET: Failure of the Ideal Dipole Approximation. Biophys. J. 2009, 96, 4779-4788.

(114) Chang, J. C. Monopole Effects on Electronic Excitation Interactions between Large Molecules. I. Application to Energy Transfer in Chlorophylls. J. Chem. Phys. 1977, 67, 3901.

(115) Pullerits, T.; Freiberg, A. Picosecond Fluorescence of Simple Photosynthetic Membranes: Evidence of Spectral Inhomogeneity and Directed Energy Transfer. Chem. Phys. 1991, 149, 409-418.

(116) Beljonne, D.; Pourtois, G.; Silva, C.; Hennebicq, E.; Herz, L. M.; Friend, R. H.; Scholes, G. D.; Setayesh, S.; Mullen, K.; Bredas, J. L.; Müllen, K.; Brédas, J. L. Interchain vs. Intrachain Energy Transfer in Acceptor-Capped Conjugated Polymers. Proc. Natl. Acad. Sci. U. S. A. 2002, 99, 10982-10987.

(117) Patwardhan, S.; Sengupta, S.; Würthner, F.; Siebbeles, L. D. A.; Grozema, F. Theoretical Study of the Optical Properties of Artificial Self-Assembled Zinc Chlorins. J. Phys. Chem. C 2010, 114, 20834-20842.

(118) Kistler, K. A.; Spano, F. C.; Matsika, S. A Benchmark of Excitonic Couplings Derived from Atomic Transition Charges. J. Phys. Chem. B 2013, 117, 20322044.

(119) Madjet, M. E.; Abdurahman, A.; Renger, T. Intermolecular Coulomb Couplings from Ab Initio Electrostatic Potentials: Application to Optical Transitions of Strongly Coupled Pigments in Photosynthetic Antennae and Reaction Centers. $J$. Phys. Chem. B 2006, 110, 17268-17281.

(120) Nagae, H.; Kakitani, T.; Katoh, T.; Mimuro, M. Calculation of the Excitation Transfer Matrix Elements between the S2 or S1 State of Carotenoid and the S2 or S1 State of Bacteriochlorophyll. J. Chem. Phys. 1993, 98, 8012. 
(121) Wong, K. F.; Bagchi, B.; Rossky, P. J. Distance and Orientation Dependence of Excitation Transfer Rates in Conjugated Systems: Beyond the Forster Theory. $J$. Phys. Chem. A 2004, 108, 5752-5763.

(122) Krueger, B. P.; Scholes, G. D.; Fleming, G. R. Calculation of Couplings and Energy-Transfer Pathways between the Pigments of LH2 by the Ab Initio Transition Density Cube Method. J. Phys. Chem. B 1998, 102, 5378-5386.

(123) Hsu, C. P.; Walla, P. J.; Head-Gordon, M.; Fleming, G. R. The Role of the S-1 State of Carotenoids in Photosynthetic Energy Transfer: The Light-Harvesting Complex II of Purple Bacteria. J. Phys. Chem. B 2001, 105, 11016-11025.

(124) Olbrich, C.; Kleinekathofer, U. Time-Dependent Atomistic View on the Electronic Relaxation in Light-Harvesting System II. J. Phys. Chem. B 2010, $114,12427-12437$.

(125) Maj, M.; Jeon, J.; Góra, R. W.; Cho, M. Induced Optical Activity of DNATemplated Cyanine Dye Aggregates: Exciton Coupling Theory and TD-DFT Studies. J. Phys. Chem. A 2013, 117, 5909-5918.

(126) Fujimoto, K. J. Electronic Coupling Calculations with Transition Charges, Dipoles, and Quadrupoles Derived from Electrostatic Potential Fitting. J. Chem. Phys. 2014, 141, 214105.

(127) Błasiak, B.; Maj, M.; Cho, M.; Góra, R. W. Distributed Multipolar Expansion Approach to Calculation of Excitation Energy Transfer Couplings. J. Chem. Theory Comput. 2015, 11, 3259-3266.

(128) Sokalski, W. A.; Poirier, R. A. Cumulative Atomic Multipole Representation of the Molecular Charge Distribution and Its Basis Set Dependence. Chem. Phys. Lett. 1983, 98, 86-92.

(129) Iozzi, M. F.; Mennucci, B.; Tomasi, J.; Cammi, R. Excitation Energy Transfer (EET) between Molecules in Condensed Matter: A Novel Application of the Polarizable Continuum Model (PCM). J. Chem. Phys. 2004, 120, 7029-7040.

(130) Muñoz-Losa, A.; Curutchet, C.; Galván, I. F.; Mennucci, B. Quantum Mechanical Methods Applied to Excitation Energy Transfer: A Comparative Analysis on Excitation Energies and Electronic Couplings. J. Chem. Phys. 2008, $129,34104$.

(131) Knox, R. S.; van Amerongen, H. Refractive Index Dependence of the Förster Resonance Excitation Transfer Rate. J. Phys. Chem. B 2002, 106, 5289-5293.

(132) Hsu, C.-P.; Fleming, G. R.; Head-Gordon, M.; Head-Gordon, T. Excitation Energy Transfer in Condensed Media. J. Chem. Phys. 2001, 114, 3065.

(133) Scholes, G. D.; Curutchet, C.; Mennucci, B.; Cammi, R.; Tomasi, J. How Solvent Controls Electronic Energy Transfer and Light Harvesting. J. Phys. Chem. B 2007, 111, 6978-6982.

(134) Curutchet, C.; Scholes, G. D.; Mennucci, B.; Cammi, R. How Solvent Controls Electronic Energy Transfer and Light Harvesting: Toward a QuantumMechanical Description of Reaction Field and Screening Effects. J. Phys. Chem. B 2007, 111, 13253-13265.

(135) Curutchet, C.; Munoz-Losa, A.; Monti, S.; Kongsted, J.; Scholes, G. D.; Mennucci, B. Electronic Energy Transfer in Condensed Phase Studied by a Polarizable QM/MM Model. J. Chem. Theory Comput. 2009, 5, 1838-1848.

(136) Curutchet, C.; Kongsted, J.; Muñoz-Losa, A.; Hossein-Nejad, H.; Scholes, G. D.; Mennucci, B. Photosynthetic Light-Harvesting Is Tuned by the Heterogeneous Polarizable Environment of the Protein. J. Am. Chem. Soc. 2011, 133, 30783084.

(137) Jurinovich, S.; Curutchet, C.; Mennucci, B. The Fenna-Matthews-Olson Protein 
Revisited: A Fully Polarizable (TD)DFT/MM Description. ChemPhysChem 2014, 15, 3194-3204.

(138) Jurinovich, S.; Viani, L.; Prandi, I. G.; Mennucci, B. Towards an Ab Initio Description of the Optical Spectra of Light-Harvesting Antennae : Application to the CP29 Complex of Photosystem II. Phys. Chem. Chem. Phys. 2015, 17, $14405-14416$.

(139) Rosnik, A. M.; Curutchet, C. Theoretical Characterization of the Spectral Density of the Water-Soluble Chlorophyll-Binding Protein from Combined Quantum Mechanics/Molecular Mechanics Molecular Dynamics Simulations. J. Chem. Theory Comput. 2015, 11, 5826-5837.

(140) König, C.; Schlüter, N.; Neugebauer, J. Direct Determination of Exciton Couplings from Subsystem Time-Dependent Density-Functional Theory within the Tamm-Dancoff Approximation. J. Chem. Phys. 2013, 138, 34104.

(141) König, C.; Neugebauer, J. Exciton Coupling Mechanisms Analyzed with Subsystem TDDFT: Direct vs Pseudo Exchange Effects. J. Phys. Chem. B 2013, 117, 3480-3487.

(142) Neugebauer, J.; Curutchet, C.; Muñoz-Losa, A.; Mennucci, B. A Subsystem TDDFT Approach for Solvent Screening Effects on Excitation Energy Transfer Couplings. J. Chem. Theory Comput. 2010, 6, 1843-1851.

(143) Albinsson, B.; Mårtensson, J.; Martensson, J.; Mårtensson, J. Excitation Energy Transfer in Donor-Bridge-Acceptor Systems. Phys. Chem. Chem. Phys. 2010, 12, 7338-7351.

(144) McConnell, H. Intramolecular Charge Transfer in Aromatic Free Radicals. $J$. Chem. Phys. 1961, 35, 508-515.

(145) Scholes, G. D.; Harcourt, R. D. Configuration Interaction and the Theory of Electronic Factors in Energy Transfer and Molecular Exciton Interactions. $J$. Chem. Phys. 1996, 104, 5054-5061.

(146) Harcourt, R. D.; Scholes, G. D.; Ghiggino, K. P. Rate Expressions for Excitation Transfer. II. Electronic Considerations of Direct and Through-configuration Exciton Resonance Interactions. J. Chem. Phys. 1994, 101, 10521.

(147) Russo, V.; Curutchet, C.; Mennucci, B. Towards a Molecular Scale Interpretation of Excitation Energy Transfer in Solvated Bichromophoric Systems. II. The through-Bond Contribution. J. Phys. Chem. B 2007, 111, 853-863.

(148) Curutchet, C.; Mennucci, B.; Scholes, G. D.; Beljonne, D. Does Forster Theory Predict the Rate of Electronic Energy Transfer for a Model Dyad at Low Temperature? J. Phys. Chem. B 2008, 112, 3759-3766.

(149) Fückel, B.; Köhn, A.; Harding, M. E.; Diezemann, G.; Hinze, G.; Basché, T.; Gauss, J. Theoretical Investigation of Electronic Excitation Energy Transfer in Bichromophoric Assemblies. J. Chem. Phys. 2008, 128, 074505.

(150) Chen, H. C.; You, Z. Q.; Hsu, C. P. The Mediated Excitation Energy Transfer: Effects of Bridge Polarizability. J. Chem. Phys. 2008, 129, 84708.

(151) Curutchet, C.; Feist, F. A.; Averbeke, B. Van; Mennucci, B.; Jacob, J.; Müllen, K.; Basché, T.; Beljonne, D. Superexchange-Mediated Electronic Energy Transfer in a Model Dyad. Phys. Chem. Chem. Phys. 2010, 12, 7378-7385.

(152) Caprasecca, S.; Curutchet, C.; Mennucci, B. Toward a Unified Modeling of Environment and Bridge-Mediated Contributions to Electronic Energy Transfer: A Fully Polarizable QM/MM/PCM Approach. J. Chem. Theory Comput. 2012, 8, 4462-4473.

(153) Hsu, C. P. The Electronic Couplings in Electron Transfer and Excitation Energy Transfer. Acc. Chem. Res. 2009, 42, 509-518. 
(154) Mead, C. A. Conditions for the Definition of a Strictly Diabatic Electronic Basis for Molecular Systems. J. Chem. Phys. 1982, 77, 6090.

(155) Van Voorhis, T.; Kowalczyk, T.; Kaduk, B.; Wang, L.-P.; Cheng, C.-L.; Wu, Q. The Diabatic Picture of Electron Transfer, Reaction Barriers, and Molecular Dynamics. Ann. Rev. Phys. Chem. 2010, 61, 149-170.

(156) Wu, Q.; Van Voorhis, T. Direct Optimization Method to Study Constrained Systems within Density-Functional Theory. Phys. Rev. A 2005, 72, 7-10.

(157) Cave, R. J.; Newton, M. D. Generalization of the Mulliken-Hush Treatment for the Calculation of Electron Transfer Matrix Elements. Chem. Phys. Lett. 1996, 249, 15-19.

(158) Hsu, C. P.; You, Z. Q.; Chen, H. C. H. Characterization of the Short-Range Couplings in Excitation Energy Transfer. J. Phys. Chem. C 2008, 112, 1204 1212.

(159) Voityuk, A. A.; Rösch, N. Fragment Charge Difference Method for Estimating Donor-Acceptor Electronic Coupling: Application to DNA Pi-Stacks. J. Chem. Phys. 2002, 117, 5607-5616.

(160) Head-Gordon, M.; Grana, A. M.; Maurice, D.; White, C. A. Analysis of Electronic Transitions as the Difference of Electron Attachment and Detachment Densities. J. Phys. Chem. 1995, 99, 14261-14270.

(161) Subotnik, J. E.; Cave, R. J.; Steele, R. P.; Shenvi, N. The Initial and Final States of Electron and Energy Transfer Processes: Diabatization as Motivated by System-Solvent Interactions. J. Chem. Phys. 2009, 130, 234102.

(162) Subotnik, J. E.; Yeganeh, S.; Cave, R. J.; Ratner, M. A. Constructing Diabatic States from Adiabatic States: Extending Generalized Mulliken-Hush to Multiple Charge Centers with Boys Localization. J. Chem. Phys. 2008, 129, 244101.

(163) Voityuk, A. A. Fragment Transition Density Method to Calculate Electronic Coupling for Excitation Energy Transfer. J. Chem. Phys. 2014, 140, 244117.

(164) Voityuk, A. A. Interaction of Dark Excited States. Comparison of Computational Approaches. J. Phys. Chem. B 2015, 119, 7417-7421.

(165) Hoyer, C. E.; Xu, X.; Ma, D.; Gagliardi, L.; Truhlar, D. G. Diabatization Based on the Dipole and Quadrupole: The DQ Method. J. Chem. Phys. 2014, 141, 114104.

(166) Closs, G. L.; Johnson, M. D.; Miller, J. R.; Piotrowiak, P. A Connection between Intramolecular Long-Range Electron, Hole, and Triplet Energy Transfers. J. Am. Chem. Soc. 1989, 111, 3751-3753.

(167) Marcus, R. A. On the Theory of Oxidation-Reduction Reactions Involving Electron Transfer. I. J. Chem. Phys. 1956, 24, 966.

(168) Place, I.; Farran, A.; Deshayes, K.; Piotrowiak, P. Triplet Energy Transfer through the Walls of Hemicarcerands: Temperature Dependence and the Role of Internal Reorganization Energy. J. Am. Chem. Soc. 1998, 120, 12626-12633.

(169) Subotnik, J. E.; Vura-Weis, J.; Sodt, A. J.; Ratner, M. A. Predicting Accurate Electronic Excitation Transfer Rates via Marcus Theory with Boys or EdmistonRuedenberg Localized Diabatization. J. Phys. Chem. A 2010, 114, 8665-8675.

(170) Landry, B. R.; Subotnik, J. E. Quantifying the Lifetime of Triplet Energy Transfer Processes in Organic Chromophores: A Case Study of 4-(2Naphthylmethyl)benzaldehyde. J. Chem. Theory Comput. 2014, 10, 4253-4263.

(171) You, Z.-Q.; Hsu, C.-P. The Fragment Spin Difference Scheme for Triplet-Triplet Energy Transfer Coupling. J. Chem. Phys. 2010, 133, 74105.

(172) You, Z.-Q.; Hsu, C.-P. Ab Inito Study on Triplet Excitation Energy Transfer in Photosynthetic Light-Harvesting Complexes. J. Phys. Chem. A 2011, 115, 4092- 
4100.

(173) Curutchet, C.; Voityuk, A. A. Triplet-Triplet Energy Transfer in DNA: A Process That Occurs on the Nanosecond Timescale. Angew. Chemie - Int. Ed. 2011, 50, 1820-1822.

(174) Voityuk, A. A. Triplet Excitation Energy Transfer through Fluorene $\pi$ Stack. $J$. Phys. Chem. C 2010, 114, 20236-20239.

(175) Yang, C.-H.; Hsu, C.-P. First-Principle Characterization for Singlet Fission Couplings. J. Phys. Chem. Lett. 2015, 6, 1925-1929.

(176) Di Valentin, M.; Tait, C. E.; Salvadori, E.; Orian, L.; Polimeno, A.; Carbonera, D. Evidence for Water-Mediated Triplet-Triplet Energy Transfer in the Photoprotective Site of the Peridinin-Chlorophyll a-Protein. Biochim. Biophys. Acta - Bioenerg. 2014, 1837, 85-97.

(177) Curutchet, C.; Voityuk, A. A. Distance Dependence of Triplet Energy Transfer in Water and Organic Solvents: A QM/MD Study. J. Phys. Chem. C 2012, 116, 22179-22185.

(178) Curutchet, C.; Voityuk, A. A. Environment Effects on Triplet-Triplet Energy Transfer in DNA. Chem. Phys. Lett. 2011, 512, 118-122.

(179) Forster, T. Zwischenmolekulare Energiewanderung Und Fluoreszenz. Ann. Phys. 1948, 2, 55-75.

(180) Braslavsky, S. E.; Fron, E.; Rodríguez, H. B.; Román, E. S.; Scholes, G. D.; Schweitzer, G.; Valeur, B.; Wirz, J. Pitfalls and Limitations in the Practical Use of Förster's Theory of Resonance Energy Transfer. Photochem. Photobiol. Sci. 2008, 7, 1444.

(181) Hennebicq, E.; Beljonne, D.; Curutchet, C.; Scholes, G. D.; Silbey, R. J. SharedMode Assisted Resonant Energy Transfer in the Weak Coupling Regime. $J$. Chem. Phys. 2009, 130, 214505.

(182) Novoderezhkin, V.; van Grondelle, R. Spectra and Dynamics in the B800 Antenna: Comparing Hierarchical Equations, Redfield and Förster Theories. $J$. Phys. Chem. B 2013, 117, 11076-11090.

(183) Engel, G. S.; Calhoun, T. R.; Read, E. L.; Ahn, T. K.; Mancal, T.; Cheng, Y. C.; Blankenship, R. E.; Fleming, G. R. Evidence for Wavelike Energy Transfer through Quantum Coherence in Photosynthetic Systems. Nature 2007, 446, 782 786.

(184) Lee, H.; Cheng, Y.-C.; Fleming, G. R. Coherence Dynamics in Photosynthesis: Protein Protection of Excitonic Coherence. Science 2007, 316, 1462-1465.

(185) Cho, M. H. Coherent Two-Dimensional Optical Spectroscopy. Chem. Rev. 2008, 108, 1331-1418.

(186) Abramavicius, D.; Palmieri, B.; Voronine, D. V.; Sanda, F.; Mukamel, S.; Šanda, F.; Mukamel, S. Coherent Multidimensional Optical Spectroscopy of Excitons in Molecular Aggregates; Quasiparticle versus Supermolecule Perspectives. Chem. Rev. 2009, 109, 2350-2408.

(187) Collini, E.; Scholes, G. D. Coherent Intrachain Energy Migration in a Conjugated Polymer at Room Temperature. Science 2009, 323, 369-373.

(188) Collini, E.; Wong, C. Y.; Wilk, K. E.; Curmi, P. M. G.; Brumer, P.; Scholes, G. D. Coherently Wired Light-Harvesting in Photosynthetic Marine Algae at Ambient Temperature. Nature 2010, 463, 644-647.

(189) Harel, E.; Engel, G. S. Quantum Coherence Spectroscopy Reveals Complex Dynamics in Bacterial Light- Harvesting Complex 2 (LH2). Proc. Natl. Acad. Sci. U.S.A. 2011, 109, 706-711.

(190) Panitchayangkoon, G.; Voronine, D. V; Abramavicius, D.; Caram, J. R.; Lewis, 
N. H. C.; Mukamel, S.; Engel, G. S. Direct Evidence of Quantum Transport in Photosynthetic Light-Harvesting Complexes. Proc. Natl. Acad. Sci. 2011, 108, 20908-20912.

(191) Schlau-Cohen, G. S.; Ishizaki, A.; Calhoun, T. R.; Ginsberg, N. S.; Ballottari, M.; Bassi, R.; Fleming, G. R. Elucidation of the Timescales and Origins of Quantum Electronic Coherence in LHCII. Nat. Chem. 2012, 4, 389-395.

(192) Turner, D. B.; Dinshaw, R.; Lee, K.-K.; Belsley, M. S.; Wilk, K. E.; Curmi, P. M. G.; Scholes, G. D. Quantitative Investigations of Quantum Coherence for a Light-Harvesting Protein at Conditions Simulating Photosynthesis. Phys. Chem. Chem. Phys. 2012, 14, 4857-4874.

(193) Wong, C. Y.; Alvey, R. M.; Turner, D. B.; Wilk, K. E.; Bryant, D. A.; Curmi, P. M. G.; Silbey, R. J.; Scholes, G. D. Electronic Coherence Lineshapes Reveal Hidden Excitonic Correlations in Photosynthetic Light Harvesting. Nat. Chem. 2012, 4, 396-404.

(194) Caram, J. R.; Lewis, N. H. C.; Fidler, A. F.; Engel, G. S. Signatures of Correlated Excitonic Dynamics in Two-Dimensional Spectroscopy of the Fenna-MatthewOlson Photosynthetic Complex. J. Chem. Phys. 2012, 136, 104505.

(195) Hildner, R.; Brinks, D.; Nieder, J. B.; Cogdell, R. J.; van Hulst, N. F. Quantum Coherent Energy Transfer over Varying Pathways in Single Light-Harvesting Complexes. Science 2013, 340, 1448-1451.

(196) Fuller, F. D.; Pan, J.; Gelzinis, A.; Butkus, V.; Senlik, S. S.; Wilcox, D. E.; Yocum, C. F.; Valkunas, L.; Abramavicius, D.; Ogilvie, J. P. Vibronic Coherence in Oxygenic Photosynthesis. Nat. Chem. 2014, 6, 706-711.

(197) Romero, E.; Augulis, R.; Novoderezhkin, V. I.; Ferretti, M.; Thieme, J.; Zigmantas, D.; van Grondelle, R. Quantum Coherence in Photosynthesis for Efficient Solar-Energy Conversion. Nat. Phys. 2014, 10, 676-682.

(198) Jun, S.; Yang, C.; Isaji, M.; Tamiaki, H.; Kim, J.; Ihee, H. Coherent Oscillations in Chlorosome Elucidated by Two-Dimensional Electronic Spectroscopy. $J$. Phys. Chem. Lett. 2014, 5, 1386-1392.

(199) McClure, S. D.; Turner, D. B.; Arpin, P. C.; Mirkovic, T.; Scholes, G. D. Coherent Oscillations in the PC577 Cryptophyte Antenna Occur in the Excited Electronic State. J. Phys. Chem. B 2014, 118, 1296-1308.

(200) Harrop, S. J.; Wilk, K. E.; Dinshaw, R.; Collini, E.; Mirkovic, T.; Teng, C. Y.; Oblinsky, D. G.; Green, B. R.; Hoef-Emden, K.; Hiller, R. G.; Scholes, G. D.; Curmi, P. M. G. Single-Residue Insertion Switches the Quaternary Structure and Exciton States of Cryptophyte Light-Harvesting Proteins. Proc. Natl. Acad. Sci. U. S. A. 2014, 111, E2666-E2675.

(201) Scholes, G. D. Quantum-Coherent Electronic Energy Transfer: Did Nature Think of It First? J. Phys. Chem. Lett. 2010, 1, 2-8.

(202) $\mathrm{Yu}, \mathrm{Z}$. G. Fluorescent Resonant Energy Transfer: Correlated Fluctuations of Donor and Acceptor. J. Chem. Phys. 2007, 127, 221101.

(203) Yu, Z. G.; Berding, M. A.; Wang, H. B. Spatially Correlated Fluctuations and Coherence Dynamics in Photosynthesis. Phys. Rev. E 2008, 78, 50902.

(204) Rebentrost, P.; Mohseni, M.; Aspuru-Guzik, A. Role of Quantum Coherence and Environmental Fluctuations in Chromophoric Energy Transport. J. Phys. Chem. B 2009, 113, 9942-9947.

(205) Nazir, A. Correlation-Dependent Coherent to Incoherent Transitions in Resonant Energy Transfer Dynamics. Phys. Rev. Lett. 2009, 103, 146404.

(206) Chin, A. W.; Datta, A.; Caruso, F.; Huelga, S. F.; Plenio, M. B. Noise-Assisted Energy Transfer in Quantum Networks and Light-Harvesting Complexes. New J. 
Phys. 2010, 12, 65002.

(207) Chen, X.; Silbey, R. J. Effect of Correlation of Local Fluctuations on Exciton Coherence. J. Chem. Phys. 2010, 132, 204503.

(208) Wu, J. L.; Liu, F.; Shen, Y.; Cao, J. S.; Silbey, R. J. Efficient Energy Transfer in Light-Harvesting Systems, I: Optimal Temperature, Reorganization Energy and Spatial-Temporal Correlations. New J. Phys. 2010, 12, 105012.

(209) Nalbach, P.; Eckel, J.; Thorwart, M. Quantum Coherent Biomolecular Energy Transfer with Spatially Correlated Fluctuations. New J. Phys. 2010, 12, 65043.

(210) Fassioli, F.; Nazir, A.; Olaya-Castro, A. Quantum State Tuning of Energy Transfer in a Correlated Environment. J. Phys. Chem. Lett. 2010, 1, 2139-2143.

(211) Hossein-Nejad, H.; Scholes, G. D. Energy Transfer, Entanglement and Decoherence in a Molecular Dimer Interacting with a Phonon Bath. New J. Phys. 2010, 12, 065045.

(212) Ishizaki, A.; Fleming, G. R. Quantum Superpositions in Photosynthetic Light Harvesting: Delocalization and Entanglement. New J. Phys. 2010, 12, 55004.

(213) Calhoun, T. R.; Fleming, G. R. Quantum Coherence in Photosynthetic Complexes. Phys. status solidi 2011, 248, 833-838.

(214) Kolli, A.; Nazir, A.; Olaya-Castro, A. Electronic Excitation Dynamics in Multichromophoric Systems Described via a Polaron-Representation Master Equation. J. Chem. Phys. 2011, 135, 154112.

(215) Strümpfer, J.; Schulten, K. The Effect of Correlated Bath Fluctuations on Exciton Transfer. J. Chem. Phys. 2011, 134, 95102.

(216) Abramavicius, D.; Mukamel, S. Exciton Dynamics in Chromophore Aggregates with Correlated Environment Fluctuations. J. Chem. Phys. 2011, 134, 1-10.

(217) Huo, P.; Coker, D. F. Influence of Environment Induced Correlated Fluctuations in Electronic Coupling on Coherent Excitation Energy Transfer Dynamics in Model Photosynthetic Systems. J. Chem. Phys. 2012, 136, 115102.

(218) Fidler, A. F.; Caram, J. R.; Hayes, D.; Engel, G. S. Towards a Coherent Picture of Excitonic Coherence in the Fenna-Matthews-Olson Complex. J. Phys. B At. Mol. Opt. Phys. 2012, 45, 154013.

(219) Vlaming, S. M.; Silbey, R. J. Correlated Intermolecular Coupling Fluctuations in Photosynthetic Complexes. J. Chem. Phys. 2012, 136, 55102.

(220) Chang, H.-T.; Cheng, Y.-C. Coherent versus Incoherent Excitation Energy Transfer in Molecular Systems. J. Chem. Phys. 2012, 137, 165103.

(221) Chin, A. W.; Huelga, S. F.; Plenio, M. B. Coherence and Decoherence in Biological Systems: Principles of Noise-Assisted Transport and the Origin of Long-Lived Coherences. Philos. Trans. R. Soc. A Math. Phys. Eng. Sci. 2012, 370, 3638-3657.

(222) Akimov, A. V.; Prezhdo, O. V. Persistent Electronic Coherence despite Rapid Loss of Electron-Nuclear Correlation. J. Phys. Chem. Lett. 2013, 4, 3857-3864.

(223) Tiwari, V.; Peters, W. K.; Jonas, D. M. Electronic Resonance with Anticorrelated Pigment Vibrations Drives Photosynthetic Energy Transfer Outside the Adiabatic Framework. Proc. Natl. Acad. Sci. U. S. A. 2013, 110, 1203-1208.

(224) Pelzer, K. M.; Fidler, A. F.; Griffin, G. B.; Gray, S. K.; Engel, G. S. The Dependence of Exciton Transport Efficiency on Spatial Patterns of Correlation within the Spectral Bath. New J. Phys. 2013, 15, 095019.

(225) Bhattacharyya, P.; Sebastian, K. L. Adiabatic Eigenfunction Based Approach to Coherent Transfer: Application to the Fenna-Matthews-Olson (FMO) Complex and the Role of Correlations in the Efficiency of Energy Transfer. J. Phys. Chem. A 2013, 117, 8806-8813. 
(226) Olbrich, C.; Strümpfer, J.; Schulten, K.; Kleinekathöfer, U. Quest for Spatially Correlated Fluctuations in the FMO Light-Harvesting Complex. J. Phys. Chem. B 2011, 115, 758-764.

(227) Shim, S.; Rebentrost, P.; Valleau, S.; Aspuru-Guzik, A. Atomistic Study of the Long-Lived Quantum Coherences in the Fenna-Matthews-Olson Complex. Biophys. J. 2012, 102, 649-660.

(228) Viani, L.; Curutchet, C.; Mennucci, B. Spatial and Electronic Correlations in the PE545 Light-Harvesting Complex. J. Phys. Chem. Lett. 2013, 4, 372-377.

(229) Wang, X.; Ritschel, G.; Sebastian, W. Open Quantum System Parameters from Molecular Dynamics. 2015, 25629-25641.

(230) Renger, T.; Klinger, A.; Steinecker, F.; Schmidt am Busch, M.; Numata, J.; Müh, F. Normal Mode Analysis of the Spectral Density of the Fenna-Matthews-Olson Light-Harvesting Protein: How the Protein Dissipates the Excess Energy of Excitons. J. Phys. Chem. B 2012, 116, 14565-14580.

(231) Kolli, A.; O’Reilly, E. J.; Scholes, G. D.; Olaya-Castro, A.; O’Reilly, E. J.; Scholes, G. D.; Olaya-Castro, A. The Fundamental Role of Quantized Vibrations in Coherent Light Harvesting by Cryptophyte Algae. J. Chem. Phys. 2012, 137, 174109.

(232) O'Reilly, E. J.; Olaya-Castro, A.; O’Reilly, E. J.; Olaya-Castro, A. NonClassicality of the Molecular Vibrations Assisting Exciton Energy Transfer at Room Temperature. Nat. Commun. 2014, 5, 3012.

(233) Rey, M. Del; Chin, A. W.; Huelga, S. F.; Plenio, M. B. Exploiting Structured Environments for Efficient Energy Transfer: The Phonon Antenna Mechanism. $J$. Phys. Chem. Lett. 2013, 4, 903-907.

(234) Chin, A. W.; Prior, J.; Rosenbach, R.; Caycedo-Soler, F.; Huelga, S. F.; Plenio, M. B. The Role of Non-Equilibrium Vibrational Structures in Electronic Coherence and Recoherence in Pigment-protein Complexes. Nat. Phys. 2013, 9, $113-118$.

(235) Gilmore, J.; McKenzie, R. H. Quantum Dynamics of Electronic Excitations in Biomolecular Chromophores: Role of the Protein Environment and Solvent. $J$. Phys. Chem. A 2008, 112, 2162-2176.

(236) Pieper, J.; Rätsep, M.; Trostmann, I.; Paulsen, H.; Renger, G.; Freiberg, A. Excitonic Energy Level Structure and Pigment-Protein Interactions in the Recombinant Water-Soluble Chlorophyll Protein. I. Difference Fluorescence Line-Narrowing. J. Phys. Chem. B 2011, 115, 4042-4052.

(237) Kell, A.; Feng, X.; Reppert, M.; Jankowiak, R. On the Shape of the Phonon Spectral Density in Photosynthetic Complexes. J. Phys. Chem. B 2013, 117, 7317-7323.

(238) Novoderezhkin, V. I.; Doust, A. B.; Curutchet, C.; Scholes, G. D.; Van Grondelle, R. Excitation Dynamics in Phycoerythrin 545: Modeling of SteadyState Spectra and Transient Absorption with Modified Redfield Theory. Biophys. J. 2010, 99, 344-352.

(239) Pachón, L. A.; Brumer, P. Direct Experimental Determination of Spectral Densities of Molecular Complexes. J. Chem. Phys. 2014, 141, 174102.

(240) Jankowiak, R.; Reppert, M.; Zazubovich, V.; Pieper, J.; Reinot, T. Site Selective and Single Complex Laser-Based Spectroscopies: A Window on Excited State Electronic Structure, Excitation Energy Transfer, and Electron-Phonon Coupling of Selected Photosynthetic Complexes. Chem. Rev. 2011, 111, 4546-4598.

(241) Renger, T.; Marcus, R. A. On the Relation of Protein Dynamics and Exciton Relaxation in Pigment-Protein Complexes: An Estimation of the Spectral Density 
and a Theory for the Calculation of Optical Spectra. J. Chem. Phys. 2002, 116, 9997-10019.

(242) Rätsep, M.; Freiberg, A. Electron-phonon and Vibronic Couplings in the FMO Bacteriochlorophyll a Antenna Complex Studied by Difference Fluorescence Line Narrowing. J. Lumin. 2007, 127, 251-259.

(243) Rätsep, M.; Pieper, J.; Irrgang, K.-D.; Freiberg, A. Excitation WavelengthDependent Electron-Phonon and Electron-Vibrational Coupling in the CP29 Antenna Complex of Green Plants. J. Phys. Chem. B 2008, 112, 110-118.

(244) Pieper, J.; Rätsep, M.; Trostmann, I.; Schmitt, F.-J.; Theiss, C.; Paulsen, H.; Eichler, H. J.; Freiberg, A.; Renger, G. Excitonic Energy Level Structure and Pigment-Protein Interactions in the Recombinant Water-Soluble Chlorophyll Protein. II. Spectral Hole-Burning Experiments. J. Phys. Chem. B 2011, 115, 4053-4065.

(245) Mercer, I. P.; Gould, I. R.; Klug, D. R. Optical Properties of Solvated Molecules Calculated by a QMMM Method - Chlorophyll a and Bacteriochlorophyll a. Faraday Discuss. 1997, 108, 51-62.

(246) Mercer, I. P.; Gould, I. R.; Klug, D. R. A Quantum Mechanical/molecular Mechanical Approach to Relaxation Dynamics: Calculation of the Optical Properties of Solvated Bacteriochlorophyll-A. J. Phys. Chem. B 1999, 103, 7720-7727.

(247) Damjanović, A.; Kosztin, I.; Kleinekathöfer, U.; Schulten, K. Excitons in a Photosynthetic Light-Harvesting System: A Combined Molecular Dynamics, Quantum Chemistry, and Polaron Model Study. Phys. Rev. E 2002, 65, 031919.

(248) Janosi, L.; Kosztin, I.; Damjanović, A. Theoretical Prediction of Spectral and Optical Properties of Bacteriochlorophylls in Thermally Disordered LH2 Antenna Complexes. J. Chem. Phys. 2006, 125, 014903.

(249) Vasil'ev, S.; Bruce, D. A Protein Dynamics Study of Photosystem II: The Effects of Protein Conformation on Reaction Center Function. Biophys. J. 2006, 90, 3062-3073.

(250) Walker, R. C.; Mercer, I. P.; Gould, I. R.; Klug, D. R. Comparison of Basis Set Effects and the Performance of Ab Initio and DFT Methods for Probing Equilibrium Fluctuations. J. Comput. Chem. 2007, 28, 478-490.

(251) Zwier, M. C.; Shorb, J. M.; Krueger, B. P. Hybrid Molecular Dynamics-Quantum Mechanics Simulations of Solute Spectral Properties in the Condensed Phase: Evaluation of Simulation Parameters. J. Comput. Chem. 2007, 28, 1572-1581.

(252) Olbrich, C.; Strumpfer, J.; Schulten, K.; Kleinekathofer, U. Theory and Simulation of the Environmental Effects on FMO Electronic Transitions. J. Phys. Chem. Lett. 2011, 2, 1771-1776.

(253) Kim, H. W.; Kelly, A.; Park, J. W.; Rhee, Y. M. All-Atom Semiclassical Dynamics Study of Quantum Coherence in Photosynthetic Fenna-MatthewsOlson Complex. J. Am. Chem. Soc. 2012, 134, 11640-11651.

(254) Valleau, S. S.; Eisfeld, A.; Aspuru-Guzik, A. A. On the Alternatives for Bath Correlators and Spectral Densities from Mixed Quantum-Classical Simulations. J. Chem. Phys. 2012, 137, 224103.

(255) Aghtar, M.; Liebers, J.; Strümpfer, J.; Schulten, K.; Kleinekathöfer, U. Juxtaposing Density Matrix and Classical Path-Based Wave Packet Dynamics. J. Chem. Phys. 2012, 136, 214101.

(256) Jing, Y. Y.; Zheng, R. H.; Li, H. X.; Shi, Q. Theoretical Study of the ElectronicVibrational Coupling in the Q(y) States of the Photosynthetic Reaction Center in Purple Bacteria. J. Phys. Chem. B 2012, 116, 1164-1171. 
(257) Rivera, E.; Montemayor, D.; Masia, M.; Coker, D. F. Influence of SiteDependent Pigment-Protein Interactions on Excitation Energy Transfer in Photosynthetic Light Harvesting. J. Phys. Chem. B 2013, 117, 5510-5521.

(258) Aghtar, M.; Strumpfer, J.; Olbrich, C.; Schulten, K.; Kleinekathöfer, U. The FMO Complex in a Glycerol-Water Mixture. J. Phys. Chem. B 2013, 117, 71577163.

(259) Aghtar, M.; Strumpfer, J.; Olbrich, C.; Schulten, K.; Kleinekathöfer, U. Different Types of Vibrations Interacting with Electronic Excitations in Phycoerythrin 545 and Fenna-Matthews-Olson Antenna Systems. J. Phys. Chem. Lett. 2014, 5, 3131-3137.

(260) Fujita, T.; Huh, J.; Saikin, S. K.; Brookes, J. C.; Aspuru-Guzik, A. Theoretical Characterization of Excitation Energy Transfer in Chlorosome Light-Harvesting Antennae from Green Sulfur Bacteria. Photosynth. Res. 2014, 120, 273-289.

(261) Viani, L.; Corbella, M.; Curutchet, C.; O’Reilly, E. J.; Olaya-Castro, A.; Mennucci, B. Molecular Basis of the Exciton-Phonon Interactions in the PE545 Light-Harvesting Complex. Phys. Chem. Chem. Phys. 2014, 16, 16302-16311.

(262) Chandrasekaran, S.; Aghtar, M.; Valleau, S.; Aspuru-Guzik, A.; Kleinekathoefer, U. Influence of Force Fields and Quantum Chemistry Approach on Spectral Densities of BChl a in Solution and in FMO Proteins. J. Phys. Chem. B 2015, 119, 9995-10004.

(263) Kim, C. W.; Park, J. W.; Rhee, Y. M. Effect of Chromophore Potential Model on the Description of Exciton-Phonon Interactions. J. Phys. Chem. Lett. 2015, 6, 2875-2880.

(264) Ambrosek, D.; Köhn, A.; Schulze, J.; Kühn, O. Quantum Chemical Parametrization and Spectroscopic Characterization of the Frenkel Exciton Hamiltonian for a J-Aggregate Forming Perylene Bisimide Dye. J. Phys. Chem. A 2012, 116, 11451-11458.

(265) Baron, R.; Gunsteren, W. F. Van; Hünenberger, P. H. Estimating the Configurational Entropy from Molecular Dynamics Simulations : Anharmonicity and Correlation Corrections to the Quasi-Harmonic Approximation. Trends Phys. Chem. 2006, 11, 87-122.

(266) Renger, T.; Marcus, R. A. Photophysical Properties of PS-2 Reaction Centers and a Discrepancy in Exciton Relaxation Times. J. Phys. Chem. B 2002, 106, 18091819.

(267) Renger, T.; Trostmann, I.; Theiss, C.; Madjet, M. E.; Richter, M.; Paulsen, H.; Eichler, H. J.; Knorr, A.; Renger, G. Refinement of a Structural Model of a Pigment-Protein Complex by Accurate Optical Line Shape Theory and Experiments. J. Phys. Chem. B 2007, 111, 10487-10501.

(268) Kubař, T.; Kleinekathöfer, U.; Elstner, M. Solvent Fluctuations Drive the Hole Transfer in DNA: A Mixed Quantum-Classical Study. J. Phys. Chem. B 2009, 113, 13107-13117.

(269) Dijkstra, A. G.; Tanimura, Y. Correlated Fluctuations in the Exciton Dynamics and Spectroscopy of DNA. New J. Phys. 2010, 12, 055005.

(270) Renger, T.; Madjet, M. E. A.; Busch, M. S. A.; Adolphs, J.; Muh, F. StructureBased Modeling of Energy Transfer in Photosynthesis. Photosynth. Res. 2013, 116, 367-388.

(271) Christensson, N.; Kauffmann, H. F.; Pullerits, T.; Mančal, T. Origin of LongLived Coherences in Light-Harvesting Complexes. J. Phys. Chem. B 2012, 116, 7449-7454.

(272) Caycedo-Soler, F.; Chin, A. W.; Almeida, J.; Huelga, S. F.; Plenio, M. B. The 
Nature of the Low Energy Band of the Fenna-Matthews-Olson Complex: Vibronic Signatures. J. Chem. Phys. 2012, 136, 155102.

(273) Wasielewski, M. R. Energy, Charge, and Spin Transport in Molecules and SelfAssembled Nanostructures Inspired by Photosynthesis. J. Org. Chem. 2006, 71, 5051-5066.

(274) Scholes, G. D.; Rumbles, G. Excitons in Nanoscale Systems. Nat. Mater. 2006, 5, 683-696.

(275) Saikin, S. K.; Eisfeld, A.; Valleau, S. S.; Aspuru-Guzik, A. A. Photonics Meets Excitonics: Natural and Artificial Molecular Aggregates. Nanophotonics 2013, 2, 21-38.

(276) Frischmann, P. D.; Mahata, K.; Würthner, F. Powering the Future of Molecular Artificial Photosynthesis with Light-Harvesting Metallosupramolecular Dye Assemblies. Chem. Soc. Rev. 2013, 42, 1847.

(277) Foster, M. E.; Azoulay, J. D.; Wong, B. M.; Allendorf, M. D. Novel Metalorganic Framework Linkers for Light Harvesting Applications. Chem. Sci. 2014, $5,2081$.

(278) Würthner, F.; Saha-Möller, C. R.; Fimmel, B.; Ogi, S.; Leowanawat, P.; Schmidt, D. Perylene Bisimide Dye Assemblies as Archetype Functional Supramolecular Materials. Chem. Rev. 2016, 116, 962-1052.

(279) Peng, H.-Q.; Niu, L.-Y.; Chen, Y.-Z.; Wu, L.-Z.; Tung, C.-H.; Yang, Q.-Z. Biological Applications of Supramolecular Assemblies Designed for Excitation Energy Transfer. Chem. Rev. 2015, 115, 7502-7542.

(280) Harriman, A. Artificial Light-Harvesting Arrays for Solar Energy Conversion. Chem. Commun. 2015, 51, 11745-11756.

(281) Kilina, S.; Kilin, D.; Tretiak, S. Light-Driven and Phonon-Assisted Dynamics in Organic and Semiconductor Nanostructures. Chem. Rev. 2015, 115, 5929-5978.

(282) Schubert, C.; Margraf, J. T.; Clark, T.; Guldi, D. M. Molecular Wires - Impact of $\pi$-Conjugation and Implementation of Molecular Bottlenecks. Chem. Soc. Rev. 2015, 44, 988-998.

(283) Wasielewski, M. R. Self-Assembly Strategies for Integrating Light Harvesting and Charge Separation in Artificial Photosynthetic Systems. Acc. Chem. Res. 2009, 42, 1910-1921.

(284) Ziessel, R.; Harriman, A. Artificial Light-Harvesting Antennae: Electronic Energy Transfer by Way of Molecular Funnels. Chem. Commun. (Camb). 2011, 47, 611-631.

(285) Balzani, V.; Bergamini, G.; Ceroni, P.; Marchi, E. Designing Light Harvesting Antennas by Luminescent Dendrimers. New J. Chem. 2011, 35, 1944.

(286) Medintz, I. L.; Mattoussi, H. Quantum Dot-Based Resonance Energy Transfer and Its Growing Application in Biology. Phys. Chem. Chem. Phys. 2009, 11, 1745 .

(287) Giannini, V.; Fernández-Domínguez, A. I.; Heck, S. C.; Maier, S. A. Plasmonic Nanoantennas: Fundamentals and Their Use in Controlling the Radiative Properties of Nanoemitters. Chem. Rev. 2011, 111, 3888-3912.

(288) Gilbert, M.; Albinsson, B. Photoinduced Charge and Energy Transfer in Molecular Wires. Chem. Soc. Rev. 2015, 44, 845-862.

(289) Caprasecca, S.; Mennucci, B. Excitation Energy Transfer in Donor-BridgeAcceptor Systems: A Combined Quantum-Mechanical/Classical Analysis of the Role of the Bridge and the Solvent. J. Phys. Chem. A 2014, 118, 6484-6491.

(290) Pettersson, K.; Kyrychenko, A.; Rönnow, E.; Ljungdahl, T.; Martensson, J.; Albinsson, B. Singlet Energy Transfer in Porphyrin-Based 
Donor-Bridge-Acceptor Systems: Interaction between Bridge Length and Bridge Energy. J. Phys. Chem. A 2006, 110, 310-318.

(291) Spiegel, J. D.; Kleinschmidt, M.; Larbig, A.; Tatchen, J.; Marian, C. M. Quantum-Chemical Studies on Excitation Energy Transfer Processes in BODIPY-Based Donor-Acceptor Systems. J. Chem. Theory Comput. 2015, 11, 4316-4327.

(292) Benniston, A. C.; Harriman, A.; Li, P.; Patel, P. V; Sams, C. A. The Effect of Torsion Angle on the Rate of Intramolecular Triplet Energy Transfer. Phys. Chem. Chem. Phys. 2005, 7, 3677-3679.

(293) Eng, M. P.; Ljungdahl, T.; Mårtensson, J.; Albinsson, B. Triplet Excitation Energy Transfer in Porphyrin-Based Donor-Bridge-Acceptor Systems with Conjugated Bridges of Varying Length: An Experimental and DFT Study. $J$. Phys. Chem. B 2006, 110, 6483-6491.

(294) Zhang, X.; Zeng, Y.; Yu, T.; Chen, J.; Yang, G.; Li, Y. Advances in Photofunctional Dendrimers for Solar Energy Conversion. J. Phys. Chem. Lett. 2014, 5, 2340-2350.

(295) Thompson, A. L.; Gaab, K. M.; Xu, J. J.; Bardeen, C. J.; Martinez, T. J. Variable Electronic Coupling in Phenylacetylene Dendrimers: The Role of Forster, Dexter, and Charge-Transfer Interactions. J. Phys. Chem. A 2004, 108, 671-682.

(296) Nelson, T.; Fernandez-Alberti, S.; Roitberg, A. E.; Tretiak, S. Nonadiabatic Excited-State Molecular Dynamics: Modeling Photophysics in Organic Conjugated Materials. Acc. Chem. Res. 2014, 47, 1155-1164.

(297) Tully, J. C. Molecular Dynamics with Electronic Transitions. J. Chem. Phys. 1990, 93, 1061.

(298) Tretiak, S.; Mukamel, S. Density Matrix Analysis and Simulation of Electronic Excitations in Conjugated and Aggregated Molecules. Chem. Rev. 2002, 102, 3171-3212.

(299) Galindo, J. F.; Atas, E.; Altan, A.; Kuroda, D. G.; Fernandez-Alberti, S.; Tretiak, S.; Roitberg, A. E.; Kleiman, V. D. Dynamics of Energy Transfer in a Conjugated Dendrimer Driven by Ultrafast Localization of Excitations. J. Am. Chem. Soc. 2015, 137, 11637-11644.

(300) Beenken, W. J. D.; Pullerits, T. Excitonic Coupling in Polythiophenes: Comparison of Different Calculation Methods. J. Chem. Phys. 2004, 120, 2490.

(301) Marguet, S.; Markovitsi, D.; Millié, P.; Sigal, H. H.; Kumar, S.; Millie, P. Influence of Disorder on Electronic Excited States: An Experimental and Numerical Study of Alkylthiotriphenylene Coluumnar Phases. J. Phys. Chem. B 1998, 102, 4697-4710.

(302) Brédas, J.-L.; Beljonne, D.; Coropceanu, V.; Cornil, J. Charge-Transfer and Energy-Transfer Processes in $\pi$-Conjugated Oligomers and Polymers: A Molecular Picture. Chem. Rev. 2004, 104, 4971-5004.

(303) Hennebicq, E.; Pourtois, G.; Scholes, G. D.; Herz, L. M.; Russell, D. M.; Silva, C.; Setayesh, S.; Grimsdale, A. C.; Müllen, K.; Brédas, J. L.; Beljonne, D. Exciton Migration in Rigid-Rod Conjugated Polymers: An Improved Forster Model. J. Am. Chem. Soc. 2005, 127, 4744-4762.

(304) Ma, H.; Qin, T.; Troisi, A. Electronic Excited States in Amorphous MEH-PPV Polymers from Large-Scale First Principles Calculations. J. Chem. Theory Comput. 2014, 10, 1272-1282.

(305) Gierschner, J.; Huang, Y. S.; Van Averbeke, B.; Cornil, J.; Friend, R. H.; Beljonne, D. Excitonic versus Electronic Couplings in Molecular Assemblies: The Importance of Non-Nearest Neighbor Interactions. J. Chem. Phys. 2009, 
$130,1-6$.

(306) Brédas, J.-L.; Norton, J. E.; Cornil, J.; Coropceanu, V. Molecular Understanding of Organic Solar Cells: The Challenges. Acc. Chem. Res. 2009, 42, 1691-1699.

(307) Cornil, J.; Verlaak, S.; Martinelli, N.; Mityashin, A.; Olivier, Y.; Van Regemorter, T.; D’Avino, G.; Muccioli, L.; Zannoni, C.; Castet, F.; Beljonne, D.; Heremans, P. Exploring the Energy Landscape of the Charge Transport Levels in Organic Semiconductors at the Molecular Scale. Acc. Chem. Res. 2013, 46, 434443.

(308) Zhugayevych, A.; Tretiak, S. Theoretical Description of Structural and Electronic Properties of Organic Photovoltaic Materials. Annu. Rev. Phys. Chem. 2015, 66, 305-330.

(309) Aragó, J.; Troisi, A. Excitonic Couplings between Molecular Crystal Pairs by a Multistate Approximation. J. Chem. Phys. 2015, 142, 164107.

(310) Aragó, J.; Troisi, A. Dynamics of the Excitonic Coupling in Organic Crystals. Phys. Rev. Lett. 2015, 114, 026402.

(311) Ziemann, D.; May, V. Distant- and Shape-Dependent Excitation Energy Transfer in Nanohybrid Systems: Computations on a Pheophorbide- $\alpha$ CdSe Nanocrystal Complex. J. Phys. Chem. Lett. 2014, 5, 1203-1209.

(312) Plehn, T.; Ziemann, D.; Megow, J.; May, V. Frenkel to Wannier-Mott Exciton Transition: Calculation of FRET Rates for a Tubular Dye Aggregate Coupled to a CdSe Nanocrystal. J. Phys. Chem. B 2015, 119, 7467-7472.

(313) Allan, G.; Delerue, C. Energy Transfer between Semiconductor Nanocrystals: Validity of Förster's Theory. Phys. Rev. B 2007, 75, 195311.

(314) Curutchet, C.; Franceschetti, A.; Zunger, A.; Scholes, G. D. Examining Förster Energy for Semiconductor Nanocrystalline Quantum Dot Donors and Acceptors. J. Phys. Chem. C 2008, 112, 13336-13341.

(315) Schrier, J.; Wang, L.-W. Shape Dependence of Resonant Energy Transfer between Semiconductor Nanocrystals. J. Phys. Chem. C 2008, 112, 11158 11161.

(316) Rebentrost, P.; Stopa, M.; Aspuru-Guzik, A. Förster Coupling in Nanoparticle Excitonic Circuits. Nano Lett. 2010, 10, 2849-2856.

(317) Austin, L. A.; Kang, B.; El-Sayed, M. A. Probing Molecular Cell Event Dynamics at the Single-Cell Level with Targeted Plasmonic Gold Nanoparticles: A Review. Nano Today 2015, 10, 542-558.

(318) Andrew, P.; Barnes, W. L. Energy Transfer across a Metal Film Mediated by Surface Plasmon Polaritons. Science 2004, 306, 1002-1005.

(319) Collini, E.; Todescato, F.; Ferrante, C.; Bozio, R.; Scholes, G. D. Photophysics and Dynamics of Surface Plasmon Polaritons-Mediated Energy Transfer in the Presence of an Applied Electric Field. J. Am. Chem. Soc. 2012, 134, 1006110070.

(320) Morton, S. M.; Silverstein, D. W.; Jensen, L. Theoretical Studies of Plasmonics Using Electronic Structure Methods. Chem. Rev. 2011, 111, 3962-3994.

(321) Jensen, L.; Aikens, C. M.; Schatz, G. C. Electronic Structure Methods for Studying Surface-Enhanced Raman Scattering. Chem. Soc. Rev. 2008, 37, 10611073.

(322) Angioni, A.; Corni, S.; Mennucci, B. Can We Control the Electronic Energy Transfer in Molecular Dyads through Metal Nanoparticles? A QM/continuum Investigation. Phys. Chem. Chem. Phys. 2013, 15, 3294-3303.

(323) Govorov, A. O.; Lee, J.; Kotov, N. A. Theory of Plasmon-Enhanced Förster Energy Transfer in Optically Excited Semiconductor and Metal Nanoparticles. 
Phys. Rev. B 2007, 76, 125308.

(324) Xie, H. Y.; Chung, H. Y.; Leung, P. T.; Tsai, D. P. Plasmonic Enhancement of Förster Energy Transfer between Two Molecules in the Vicinity of a Metallic Nanoparticle: Nonlocal Optical Effects. Phys. Rev. B 2009, 80, 155448.

(325) Gersten, J. I.; Nitzan, A. Accelerated Energy Transfer between Molecules near a Solid Particle. Chem. Phys. Lett. 1984, 104, 31-37.

(326) Hua, X. M.; Gersten, J. I.; Nitzan, A. Theory of Energy Transfer between Molecules near Solid State Particles. J. Chem. Phys. 1985, 83, 3650.

(327) Andreussi, O.; Biancardi, A.; Corni, S.; Mennucci, B. Plasmon-Controlled LightHarvesting: Design Rules for Biohybrid Devices via Multiscale Modeling. Nano Lett. 2013, 13, 4475-4484.

(328) Andreussi, O.; Caprasecca, S.; Cupellini, L.; Guarnetti-Prandi, I.; Guido, C. A.; Jurinovich, S.; Viani, L.; Mennucci, B. Plasmon Enhanced Light Harvesting: Multiscale Modeling of the FMO Protein Coupled with Gold Nanoparticles. $J$. Phys. Chem. A 2015, 119, 5197-5206.

(329) Masiello, D. J.; Schatz, G. C. Many-Body Theory of Surface-Enhanced Raman Scattering. Phys. Rev. A 2008, 78, 042505.

(330) Kyas, G.; May, V. Density Matrix Based Microscopic Theory of Molecule Metal-Nanoparticle Interactions: Linear Absorbance and Plasmon Enhancement of Intermolecular Excitation Energy Transfer. J. Chem. Phys. 2011, 134, 1-11.

(331) Cogdell, R. J.; Gall, A.; Kohler, J. The Architecture and Function of the LightHarvesting Apparatus of Purple Bacteria: From Single Molecules to in Vivo Membranes. Q. Rev. Biophys. 2006, 39, 227-324.

(332) van Grondelle, R.; Novoderezhkin, V. I. Energy Transfer in Photosynthesis: Experimental Insights and Quantitative Models. Phys. Chem. Chem. Phys. 2006, 8, 793-807.

(333) McLuskey, K.; Prince, S. M.; Cogdell, R. J.; Isaacs, N. W. The Crystallographic Structure of the B800-820 LH3 Light-Harvesting Complex from the Purple Bacteria Rhodopseudomonas Acidophila Strain 7050. Biochemistry 2001, 40, 8783-8789.

(334) McDermott, G.; Prince, S. M.; Freer, A. A.; Hawthornthwaite-Lawless, A. M.; Papiz, M. Z.; Cogdell, R. J.; Isaacs, N. W. Crystal Structure of an Integral Membrane Light-Harvesting Complex from Photosynthetic Bacteria. Nature 1995, 374, 517-521.

(335) Prince, S. M.; Papiz, M. Z.; Freer, A. A.; McDermott, G.; HawthornthwaiteLawless, A. M.; Cogdell, R. J.; Isaacs, N. W. Apoprotein Structure in the LH2 Complex from Rhodopseudomonas Acidophila Strain 10050: Modular Assembly and Protein Pigment Interactions. J. Mol. Biol. 1997, 268, 412-423.

(336) Papiz, M. Z.; Prince, S. M.; Howard, T.; Cogdell, R. J.; Isaacs, N. W. The Structure and Thermal Motion of the B800-850 LH2 Complex from Rps.acidophila at 2.0 ̊ Resolution and 100K: New Structural Features and Functionally Relevant Motions. J. Mol. Biol. 2003, 326, 1523-1538.

(337) Koepke, J.; Hu, X.; Muenke, C.; Schulten, K.; Michel, H. The Crystal Structure of the Light-Harvesting Complex II (B800-850) from Rhodospirillum Molischianum. Structure 1996, 4, 581-597.

(338) Roszak, A. W.; Howard, T. D.; Southall, J.; Gardiner, A. T.; Law, C. J.; Isaacs, N. W.; Cogdell, R. J. Crystal Structure of the RC-LH1 Core Complex from Rhodopseudomonas Palustris. Science 2003, 302, 1969-1972.

(339) Cherezov, V.; Clogston, J.; Papiz, M. Z.; Caffrey, M. Room to Move: Crystallizing Membrane Proteins in Swollen Lipidic Mesophases. J. Mol. Biol. 
2006, 357, 1605-1618.

(340) Sundstrom, V.; Pullerits, T.; van Grondelle, R. Photosynthetic Light-Harvesting: Reconciling Dynamics and Structure of Purple Bacterial LH2 Reveals Function of Photosynthetic Unit. J. Phys. Chem. B 1999, 103, 2327-2346.

(341) Scholes, G. D.; Gould, I. R.; Cogdell, R. J.; Fleming, G. R. Ab Initio Molecular Orbital Calculations of Electronic Couplings in the LH2 Bacterial LightHarvesting Complex of Rps-Acidophila. J. Phys. Chem. B 1999, 103, 25432553.

(342) He, Z.; Sundström, V.; Pullerits, T. Influence of the Protein Binding Site on the Excited States of Bacteriochlorophyll: DFT Calculations of B800 in LH2. J. Phys. Chem. B 2002, 106, 11606-11612.

(343) Neugebauer, J. Photophysical Properties of Natural Light-Harvesting Complexes Studied by Subsystem Density Functional Theory. J. Phys. Chem. B 2008, 112, 2207-2217.

(344) Sauer, K.; Cogdell, R. J.; Prince, S. M.; Freer, A.; Isaacs, N. W.; Scheer, H. Structure-Based Calculations of the Optical Spectra of the LH2 Bacteriochlorophyll-Protein Complex from Rhodopseudomonas Acidophila. Photochem. Photobiol. 1996, 64, 564-576.

(345) Alden, R. G.; Johnson, E.; Nagarajan, V.; Parson, W. W.; Law, C. J.; Cogdell, R. G. Calculations of Spectroscopic Properties of the LH2 Bacteriochlorophyll Protein Antenna Complex from Rhodopseudomonas Acidophila. J. Phys. Chem. $B$ 1997, 101, 4667-4680.

(346) Hu, X.; Ritz, T.; Damjanović, A.; Schulten, K. Pigment Organization and Transfer of Electronic Excitation in the Photosynthetic Unit of Purple Bacteria. $J$. Phys. Chem. B 1997, 101, 3854-3871.

(347) Cory, M. G.; Zerner, M. C.; Hu, X.; Schulten, K. Electronic Excitations in Aggregates of Bacteriochlorophylls. J. Phys. Chem. B 1998, 102, 7640-7650.

(348) Hu, X.; Damjanović, A.; Ritz, T.; Schulten, K. Architecture and Mechanism of the Light-Harvesting Apparatus of Purple Bacteria. Proc. Natl. Acad. Sci. U. S. A. 1998, 95, 5935-5941.

(349) Krueger, B. P.; Scholes, G. D.; Jimenez, R.; Fleming, G. R. Electronic Excitation Transfer from Carotenoid to Bacteriochlorophyll in the Purple Bacterium Rhodopseudomonas Acidophila. J. Phys. Chem. B 1998, 102, 2284-2292.

(350) P. Krueger, B.; D. Scholes, G.; R. Gould, I.; R. Fleming, G. Carotenoid Mediated B800-B850 Coupling in LH2. PhysChemComm 1999, 2, 34.

(351) Linnanto, J.; Korppi-Tommopa, J. E. I.; Helenius, V. M. Electronic States, Absorption Spectrum and Circular Dichroism Spectrum of the Photosynthetic Bacterial LH2 Antenna of Rhodopseudomonas Acidophila as Predicted by Exciton Theory and Semiempirical Calculations. J. Phys. Chem. B 1999, 103, 8739-8750.

(352) Ihalainen, J. A.; Linnanto, J.; Myllyperkio, P.; van Stokkum, I. H. M.; Ucker, B.; Scheer, H.; Korppi-Tommola, J. E. I. Energy Transfer in LH2 of Rhodospirillum Molischianum, Studied by Subpicosecond Spectroscopy and Configuration Interaction Exciton Calculations. J. Phys. Chem. B 2001, 105, 9849-9856.

(353) Tretiak, S.; Middleton, C.; Chernyak, V.; Mukamel, S. Exciton Hamiltonian for the Bacteriochlorophyll System in the LH2 Antenna Complex of Purple Bacteria. J. Phys. Chem. B 2000, 104, 4519-4528.

(354) Tretiak, S.; Middleton, C.; Chernyak, V.; Mukamel, S. Bacteriochlorophyll and Carotenoid Excitonic Couplings in the LH2 System of Purple Bacteria. J. Phys. Chem. B 2000, 104, 9540-9553. 
(355) Zhang, J.-P.; Fujii, R.; Qian, P.; Inaba, T.; Mizoguchi, T.; Koyama, Y.; Onaka, K.; Watanabe, Y.; Nagae, H. Mechanism of the Carotenoid-to-

Bacteriochlorophyll Energy Transfer via the S1 State in the LH2 Complexes from Purple Bacteria. J. Phys. Chem. B 2000, 104, 3683-3691.

(356) Walla, P. J.; Linden, P. A.; Hsu, C. P.; Scholes, G. D.; Fleming, G. R.

Femtosecond Dynamics of the Forbidden Carotenoid S1 State in Light-

Harvesting Complexes of Purple Bacteria Observed after Two-Photon Excitation.

Proc. Natl. Acad. Sci. U. S. A. 2000, 97, 10808-10813.

(357) Ritz, T.; Schulten, K.; Damjanovic, A.; Schulten, K.; Zhang, J.; Koyama, Y.

Efficient Light Harvesting through Carotenoids. Photosynth. Res. 2000, 66, 125144.

(358) Scholes, G. D.; Fleming, G. R. On the Mechanism of Light Harvesting in Photosynthetic Purple Bacteria: B800 to B850 Energy Transfer. J. Phys. Chem. B 2000, 104, 1854-1868.

(359) Jang, S.; Newton, M. D.; Silbey, R. J. Multichromophoric Förster Resonance Energy Transfer from B800 to B850 in the Light Harvesting Complex 2:

Evidence for Subtle Energetic Optimization by Purple Bacteria. J. Phys. Chem. B 2007, 111, 6807-6814.

(360) Olbrich, C.; Liebers, J.; Kleinekathöfer, U. Modeling of Light-Harvesting in Purple Bacteria Using a Time-Dependent Hamiltonian Approach. Phys. Status Solidi 2011, 248, 393-398.

(361) van der Vegte, C. P.; Prajapati, J. D.; Kleinekathoefer, U.; Knoester, J.; Jansen, T. L. C. Atomistic Modeling of Two-Dimensional Electronic Spectra and Excited State Dynamics for a Light Harvesting 2 Complex. J. Phys. Chem. B 2015, 119, 1302-1313.

(362) Linnanto, J.; Freiberg, A.; Korppi-Tommola, J. Quantum Chemical Simulations of Excited-State Absorption Spectra of Photosynthetic Bacterial Reaction Center and Antenna Complexes. J. Phys. Chem. B 2011, 115, 5536-5544.

(363) Rancova, O.; Sulskus, J.; Abramavicius, D. Insight into the Structure of Photosynthetic LH2 Aggregate from Spectroscopy Simulations. J. Phys. Chem. B 2012, 116, 7803-7814.

(364) Chmeliov, J.; Songaila, E.; Rancova, O.; Gall, A.; Robert, B.; Abramavicius, D.; Valkunas, L. Excitons in the LH3 Complexes from Purple Bacteria. J. Phys. Chem. B 2013, 117, 11058-11068.

(365) Sener, M. K.; Olsen, J. D.; Hunter, C. N.; Schulten, K. Atomic-Level Structural and Functional Model of a Bacterial Photosynthetic Membrane Vesicle. Proc. Natl. Acad. Sci. U. S. A. 2007, 104, 15723-15728.

(366) Chandler, D. E.; Strümpfer, J.; Sener, M.; Scheuring, S.; Schulten, K. Light Harvesting by Lamellar Chromatophores in Rhodospirillum Photometricum. Biophys. J. 2014, 106, 2503-2510.

(367) Shrestha, K.; Jakubikova, E. The Ground State Electronic Structure of RC-LH1 and LH2 Pigment Assemblies of Purple Bacteria via the EBF-MO Method. $J$. Phys. Chem. A 2015, 119, 8934-8943.

(368) Jang, S.; Rivera, E.; Montemayor, D. Molecular Level Design Principle behind Optimal Sizes of Photosynthetic LH2 Complex: Taming Disorder through Cooperation of Hydrogen Bonding and Quantum Delocalization. J. Phys. Chem. Lett. 2015, 928-934.

(369) Oostergetel, G. T.; van Amerongen, H.; Boekema, E. J. The Chlorosome: A Prototype for Efficient Light Harvesting in Photosynthesis. Photosynth. Res. 2010, 104, 245-255. 
(370) Ganapathy, S.; Oostergetel, G. T.; Wawrzyniak, P. K.; Reus, M.; Gomez Maqueo Chew, A.; Buda, F.; Boekema, E. J.; Bryant, D. a; Holzwarth, A. R.; de Groot, H. J. M. Alternating Syn-Anti Bacteriochlorophylls Form Concentric Helical Nanotubes in Chlorosomes. Proc. Natl. Acad. Sci. U. S. A. 2009, 106, 85258530.

(371) Olson, J. M. The FMO Protein. Photosynth. Res. 2004, 80, 181-187.

(372) Milder, M. T. W.; Bruggemann, B.; van Grondelle, R.; Herek, J. L. Revisiting the Optical Properties of the FMO Protein. Photosynth. Res. 2010, 104, 257-274.

(373) Tronrud, D. E.; Schmid, M. F.; Matthews, B. W. Structure and X-Ray Amino Acid Sequence of a Bacteriochlorophyll a Protein from Prosthecochloris Aestuarii Refined at $1.9 \AA$ Resolution. J. Mol. Biol. 1986, 188, 443-454.

(374) Tronrud, D. E.; Wen, J.; Gay, L.; Blankenship, R. E. The Structural Basis for the Difference in Absorbance Spectra for the FMO Antenna Protein from Various Green Sulfur Bacteria. Photosynth. Res. 2009, 100, 79-87.

(375) Schmidt am Busch, M.; Müh, F.; El-Amine Madjet, M.; Renger, T. The Eighth Bacteriochlorophyll Completes the Excitation Energy Funnel in the FMO Protein. J. Phys. Chem. Lett. 2011, 2, 93-98.

(376) Pearlstein, R. M.; Hemenger, R. P. Bacteriochlorophyll Electronic Transition Moment Directions in Bacteriochlorophyll a-Protein. Proc. Natl. Acad. Sci. U. S. A. 1978, 75, 4920-4924.

(377) Gudowska-Nowak, E.; Newton, M. D.; Fajer, J. Conformational and Environmental Effects on Bacteriochlorophyll Optical Spectra: Correlations of Calculated Spectra with Structural Results. J. Phys. Chem. 1990, 94, 5795-5801.

(378) Adolphs, J.; Renger, T. How Proteins Trigger Excitation Energy Transfer in the FMO Complex of Green Sulfur Bacteria. Biophys. J. 2006, 91, 2778-2797.

(379) Wen, J.; Zhang, H.; Gross, M. L.; Blankenship, R. E. Membrane Orientation of the FMO Antenna Protein from Chlorobaculum Tepidum as Determined by Mass Spectrometry-Based Footprinting. Proc. Natl. Acad. Sci. U. S. A. 2009, 106, 6134-6139.

(380) Olbrich, C.; Jansen, T. L. C.; Liebers, J.; Aghtar, M.; Strumpfer, J.; Schulten, K.; Knoester, J.; Kleinekathofer, U. From Atomistic Modeling to Excitation Transfer and Two-Dimensional Spectra of the FMO Light-Harvesting Complex. J. Phys. Chem. B 2011, 115, 8609-8621.

(381) Gao, J.; Shi, W.; Ye, J.; Wang, X.; Hirao, H.; Zhao, Y. QM/MM Modeling of Environmental Effects on Electronic Transitions of the FMO Complex. J. Phys. Chem. B 2013, 117, 3488-3495.

(382) Cole, D. J.; Chin, A. W.; Hine, N. D. M.; Haynes, P. D.; Payne, M. C. Toward Ab Initio Optical Spectroscopy of the Fenna-Matthews-Olson Complex. J. Phys. Chem. Lett. 2013, 4, 4206-4212.

(383) Ishizaki, A.; Fleming, G. R. Theoretical Examination of Quantum Coherence in a Photosynthetic System at Physiological Temperature. Proc. Natl. Acad. Sci. U. S. A. 2009, 106, 17255-17260.

(384) Panitchayangkoon, G.; Hayes, D.; Fransted, K. A.; Caram, J. R.; Harel, E.; Wen, J. Z.; Blankenship, R. E.; Engel, G. S. Long-Lived Quantum Coherence in Photosynthetic Complexes at Physiological Temperature. Proc. Natl. Acad. Sci. U. S. A. 2010, 107, 12766-12770.

(385) Gillis, C. G.; Jones, G. A. A Theoretical Investigation into the Effects of Temperature on Spatiotemporal Dynamics of EET in the FMO Complex. J. Phys. Chem. B 2015, 119, 4165-4174.

(386) Fujita, T.; Brookes, J. C.; Saikin, S. K.; Aspuru-Guzik, A. Memory-Assisted 
Exciton Diffusion in the Chlorosome Light-Harvesting Antenna of Green Sulfur Bacteria. J. Phys. Chem. Lett. 2012, 3, 2357-2361.

(387) Sawaya, N. P. D.; Huh, J.; Fujita, T.; Saikin, S. K.; Aspuru-Guzik, A. Fast Delocalization Leads To Robust Long-Range Excitonic Transfer in a Large Quantum Chlorosome Model. Nano Lett. 2015, 15, 1722-1729.

(388) Huh, J.; Saikin, S. K.; Brookes, J. C.; Valleau, S.; Fujita, T.; Aspuru-Guzik, A. Atomistic Study of Energy Funneling in the Light-Harvesting Complex of Green Sulfur Bacteria. J. Am. Chem. Soc. 2014, 136, 2048-2057.

(389) Su, H.-N.; Xie, B.-B.; Zhang, X.-Y.; Zhou, B.-C.; Zhang, Y.-Z. The Supramolecular Architecture, Function, and Regulation of Thylakoid Membranes in Red Algae: An Overview. Photosynth. Res. 2010, 106, 73-87.

(390) Scharnagl, C.; Schneider, S. UV-Visible Absorption and Circular Dichroism Spectra of the Subunits of C-Phycocyanin I: Quantitative Assessment of the Effect of Chromophore - protein Interaction in the $\alpha$-Subunit. J. Photochem. Photobiol. B Biol. 1989, 3, 603-614.

(391) Scharnagl, C.; Schneider, S. UV-Visible Absorption and Circular Dichroism Spectra of the Subunits of C-Phycocyanin II: A Quantitative Discussion of the Chromophore - protein and Chromophore - chromophore Interactions in the $\beta$ Subunit. J. Photochem. Photobiol. B Biol. 1991, 8, 129-157.

(392) Scharnagl, C.; Fischer, S. F. Reversible Photochemistry in the $\alpha$-Subunit of Phycoerythrocyanin: Characterization of Chromophore and Protein by Molecular Dynamics and Quantum Chemical Calculations. Photochem. Photobiol. 1993, $57,63-70$.

(393) Kikuchi, H.; Sugimoto, T.; Mimuro, M. An Electronic State of the Chromophore, Phycocyanobilin, and Its Interaction with the Protein Moiety in C-Phycocyanin: Protonation of the Chromophore. Chem. Phys. Lett. 1997, 274, 460-465.

(394) Wan, J.; Xu, X.; Ren, Y.; Yang, G. A Time Dependent Density Functional Theory Study of Alpha-84 Phycocyanobilin Chromophore in C-Phycocyanin. $J$. Phys. Chem. B 2005, 109, 11088-11090.

(395) Ren, Y.; Wan, J.; Xu, X.; Zhang, Q.; Yang, G. A Time-Dependent Density Functional Theory Investigation of the Spectroscopic Properties of the BetaSubunit in C-Phycocyanin. J. Phys. Chem. B 2006, 110, 18665-18669.

(396) Zazza, C.; Sanna, N.; Aschi, M. Theoretical Study of Alpha-84 Phycocyanobilin Chromophore from the Thermophilic Cyanobacterium Synechococcus Elongatus. J. Phys. Chem. B 2007, 111, 5596-5601.

(397) Womick, J. M.; Liu, H.; Moran, A. M. Exciton Delocalization and Energy Transport Mechanisms in R-Phycoerythrin. J. Phys. Chem. A 2011, 115, 24712482.

(398) Womick, J. M.; West, B. A.; Scherer, N. F.; Moran, A. M. Vibronic Effects in the Spectroscopy and Dynamics of C -Phycocyanin. J. Phys. B At. Mol. Opt. Phys. 2012, 45, 154016.

(399) Ren, Y.; Chi, B.; Melhem, O.; Wei, K.; Feng, L.; Li, Y.; Han, X.; Li, D.; Zhang, Y.; Wan, J.; Xu, X.; Yang, M. Understanding the Electronic Energy Transfer Pathways in the Trimeric and Hexameric Aggregation State of Cyanobacteria Phycocyanin within the Framework of Förster Theory. J. Comput. Chem. 2013, $34,1005-1012$.

(400) Doust, A. B.; Marai, C. N. J.; Harrop, S. J.; Wilk, K. E.; Curmi, P. M. G.; Scholes, G. D. Developing a Structure-Function Model for the Cryptophyte Phycoerythrin 545 Using Ultrahigh Resolution Crystallography and Ultrafast Laser Spectroscopy. J. Mol. Biol. 2004, 344, 135-153. 
(401) Doust, A. B.; Wilk, K. E.; Curmi, P. M. G.; Scholes, G. D. The Photophysics of Cryptophyte Light-Harvesting. J. Photochem. Photobiol. a-Chemistry 2006, 184, $1-17$.

(402) Mirkovic, T.; Doust, A. B.; Kim, J.; Wilk, K. E.; Curutchet, C.; Mennucci, B.; Cammi, R.; Curmi, P. M. G.; Scholes, G. D. Ultrafast Light Harvesting Dynamics in the Cryptophyte Phycocyanin 645. Photochem. Photobiol. Sci. 2007, 6, 964-975.

(403) Curutchet, C.; Novoderezhkin, V. I.; Kongsted, J.; Muñoz-Losa, A.; Van Grondelle, R.; Scholes, G. D.; Mennucci, B. Energy Flow in the Cryptophyte PE545 Antenna Is Directed by Bilin Pigment Conformation. J. Phys. Chem. B 2013, 117, 4263-4273.

(404) Hossein-Nejad, H.; Curutchet, C.; Kubica, A.; Scholes, G. D. DelocalizationEnhanced Long-Range Energy Transfer between Cryptophyte Algae PE545 Antenna Proteins. J. Phys. Chem. B 2011, 115, 5243-5253.

(405) Aghtar, M.; Kleinekathöfer, U. Environmental Coupling and Population Dynamics in the PE545 Light-Harvesting Complex. J. Lumin. 2016, 169, 406409.

(406) Falciatore, A.; Bowler, C. Revealing the Molecular Sectrets of Marine Diatoms. Annu. Rev. Plant Biol. 2002, 53, 109-130.

(407) Premvardhan, L.; Robert, B.; Beer, A.; Büchel, C. Pigment Organization in Fucoxanthin Chlorophyll a/c2 Proteins (FCP) Based on Resonance Raman Spectroscopy and Sequence Analysis. Biochim. Biophys. Acta-Bioenerg. 2010, 1797, 1647-1656.

(408) Papagiannakis, E.; van Stokkum, I. H. M.; Fey, H.; Büchel, C.; Van Grondelle, R. Spectroscopic Characterization of the Excitation Energy Transfer in the Fucoxanthinchlorophyll Protein of Diatoms. Photosynth. Res. 2005, 86, 241-250.

(409) Kosumi, D.; Kita, M.; Fujii, R.; Sugisaki, M.; Oka, N.; Takaesu, Y.; Taira, T.; Iha, M.; Hashimoto, H. Excitation Energy-Transfer Dynamics of Brown Algal Photosynthetic Antennas. J. Phys. Chem. Lett. 2012, 3, 2659-2664.

(410) Songaila, E.; Augulis, R.; Gelzinis, A.; Butkus, V.; Gall, A.; Büchel, C.; Robert, B.; Zigmantas, D.; Abramavicius, D.; Valkunas, L. Ultrafast Energy Transfer from Chlorophyll c 2 to Chlorophyll a in Fucoxanthin-Chlorophyll Protein Complex. J. Phys. Chem. Lett. 2013, 4, 3590-3595.

(411) Nagao, R.; Yokono, M.; Teshigahara, A.; Akimoto, S.; Tomo, T. LightHarvesting Ability of the Fucoxanthin Chlorophyll A/c-Binding Protein Associated with Photosystem II from the Diatom Chaetoceros Gracilis as Revealed by Picosecond Time-Resolved Fluorescence Spectroscopy. J. Phys. Chem. B 2014, 118, 5093-5100.

(412) Nagao, R.; Yokono, M.; Tomo, T.; Akimoto, S. Control Mechanism of Excitation Energy Transfer in a Complex Consisting of Photosystem II and Fucoxanthin Chlorophyll a / c -Binding Protein. J. Phys. Chem. Lett. 2014, 5, 2983-2987.

(413) Butkus, V.; Gelzinis, A.; Augulis, R.; Gall, A.; Büchel, C.; Robert, B.; Zigmantas, D.; Valkunas, L.; Abramavicius, D. Coherence and Population Dynamics of Chlorophyll Excitations in FCP Complex: Two-Dimensional Spectroscopy Study. J. Chem. Phys. 2015, 142, 212414.

(414) Gelzinis, A.; Butkus, V.; Songaila, E.; Augulis, R.; Gall, A.; Büchel, C.; Robert, B.; Abramavicius, D.; Zigmantas, D.; Valkunas, L. Mapping Energy Transfer Channels in Fucoxanthin-Chlorophyll Protein Complex. Biochim. Biophys. Acta 2015, 1847, 241-247. 
(415) Premvardhan, L.; Sandberg, D. J.; Fey, H.; Birge, R. R.; Büchel, C.; Van Grondelle, R. The Charge-Transfer Properties of the S2 State of Fucoxanthin in Solution and in Fucoxanthin Chlorophyll-a/c2 Protein (FCP) Based on Stark Spectroscopy and Molecular-Orbital Theory. J. Phys. Chem. B 2008, 112, $11838-11853$.

(416) Di Valentin, M.; Meneghin, E.; Orian, L.; Polimeno, A.; Büchel, C.; Salvadori, E.; Kay, C. W. M.; Carbonera, D. Triplet-Triplet Energy Transfer in Fucoxanthin-Chlorophyll Protein from Diatom Cyclotella Meneghiniana: Insights into the Structure of the Complex. Biochim. Biophys. Acta-Bioenerg. 2013, 1827, 1226-1234.

(417) Hofmann, E.; Wrench, P. M.; Sharples, F. P.; Hiller, R. G.; Welte, W.; Diederichs, K. Structural Basis of Light Harvesting by Carotenoids: PeridininChlorophyll-Protein from Amphidinium Carterae. Science 1996, 272, 17881791.

(418) Polivka, T.; Hiller, R. G.; Frank, H. A. Spectroscopy of the PeridininChlorophyll-a Protein: Insight into Light-Harvesting Strategy of Marine Algae. Arch. Biochem. Biophys. 2007, 458, 111-120.

(419) Bricker, W. P.; Lo, C. S. Excitation Energy Transfer in the Peridinin-Chlorophyll a-Protein Complex Modeled Using Configuration Interaction. J. Phys. Chem. B 2014, 118, 9141-9154.

(420) Bricker, W. P.; Lo, C. S. Efficient Pathways of Excitation Energy Transfer from Delocalized S 2 Excitons in the Peridinin-Chlorophyll a-Protein Complex. $J$. Phys. Chem. B 2015, 119, 5755-5764.

(421) Carbonera, D.; Giacometti, G.; Segre, U.; Hofmann, E.; Hiller, R. G. StructureBased Calculations of the Optical Spectra of the Light-Harvesting PeridininChlorophyll-Protein Complexes from Amphidinium Carterae and Heterocapsa Pygmaea. J. Phys. Chem. B 1999, 103, 6349-6356.

(422) Ilagan, R. P.; Koscielecki, J. F.; Hiller, R. G.; Sharples, F. P.; Gibson, G. N.; Birge, R. R.; Frank, H. A. Femtosecond Time-Resolved Absorption Spectroscopy of Main-Form and High-Salt Peridinin-Chlorophyll a -Proteins at Low Temperatures. Biochemistry 2006, 45, 14052-14063.

(423) Di Valentin, M.; Ceola, S.; Agostini, G.; Giacometti, G. M.; Angerhofer, A.; Crescenzi, O.; Barone, V.; Carbonera, D. Pulse ENDOR and Density Functional Theory on the Peridinin Triplet State Involved in the Photo-Protective Mechanism in the Peridinin-Chlorophyll a-Protein from Amphidinium Carterae. Biochim. Biophys. Acta 2008, 1777, 295-307.

(424) Zigmantas, D.; Hiller, R. G.; Sundstrom, V.; Polivka, T. Carotenoid to Chlorophyll Energy Transfer in the Peridinin-Chlorophyll-a-Protein Complex Involves an Intramolecular Charge Transfer State. Proc. Natl. Acad. Sci. U. S. A. 2002, 99, 16760-16765.

(425) Shima, S.; Ilagan, R. P.; Gillespie, N.; Sommer, B. J.; Hiller, R. G.; Sharples, F. P.; Frank, H. A.; Birge, R. R. Two-Photon and Fluorescence Spectroscopy and the Effect of Environment on the Photochemical Properties of Peridinin in Solution and in the Peridinin-Chlorophyll-Protein from Amphidinium Carterae. J. Phys. Chem. A 2003, 107, 8052-8066.

(426) Vaswani, H. M.; Hsu, C. P.; Head-Gordon, M.; Fleming, G. R. Quantum Chemical Evidence for an Intramolecular Charge-Transfer State in the Carotenoid Peridinin of Peridinin-Chlorophyll-Protein. J. Phys. Chem. B 2003, 107, 7940-7946.

(427) Wagner, N. L.; Greco, J. A.; Enriquez, M. M.; Frank, H. A.; Birge, R. R. The 
Nature of the Intramolecular Charge Transfer State in Peridinin. Biophys. J. 2013, 104, 1314-1325.

(428) Qin, X.; Suga, M.; Kuang, T.; Shen, J.-R. Structural Basis for Energy Transfer Pathways in the Plant PSI-LHCI Supercomplex. Science 2015, 348, 989-995.

(429) Liu, Z. F.; Yan, H. C.; Wang, K. B.; Kuang, T. Y.; Zhang, J. P.; Gui, L. L.; An, X. M.; Chang, W. R. Crystal Structure of Spinach Major Light-Harvesting Complex at 2.72 Angstrom Resolution. Nature 2004, 428, 287-292.

(430) Standfuss, R.; van Scheltinga, A. C. T.; Lamborghini, M.; Kuhlbrandt, W. Mechanisms of Photoprotection and Nonphotochemical Quenching in Pea LightHarvesting Complex at 2.5A Resolution. Embo J. 2005, 24, 919-928.

(431) Pan, X.; Liu, Z.; Li, M.; Chang, W. Architecture and Function of Plant LightHarvesting Complexes II. Curr. Opin. Struct. Biol. 2013, 23, 1-11.

(432) Pan, X.; Li, M.; Wan, T.; Wang, L.; Jia, C.; Hou, Z.; Zhao, X.; Zhang, J.; Chang, W. Structural Insights into Energy Regulation of Light-Harvesting Complex CP29 from Spinach. Nat. Struct. Mol. Biol. 2011, 18, 309-315.

(433) Umena, Y.; Kawakami, K.; Shen, J.-R.; Kamiya, N. Crystal Structure of OxygenEvolving Photosystem II at a Resolution of $1.9 \AA$. Nature 2011, 473, 55-60.

(434) Niyogi, K. K.; Truong, T. B. Evolution of Flexible Non-Photochemical Quenching Mechanisms That Regulate Light Harvesting in Oxygenic Photosynthesis. Curr. Opin. Plant Biol. 2013, 16, 307-314.

(435) Schlau-Cohen, G. S.; Calhoun, T. R.; Ginsberg, N. S.; Read, E. L.; Ballottari, M.; Bassi, R.; van Grondelle, R.; Fleming, G. R. Pathways of Energy Flow in LHCII from Two-Dimensional Electronic Spectroscopy. J. Phys. Chem. B 2009, 113, 15352-15363.

(436) Frahmcke, J. S.; Walla, P. J.; Frähmcke, J. S.; Walla, P. J. Coulombic Couplings between Pigments in the Major Light-Harvesting Complex LHC II Calculated by the Transition Density Cube Method. Chem. Phys. Lett. 2006, 430, 397-403.

(437) Linnanto, J.; Martiskainen, J.; Lehtovuori, V.; Ihalainen, J.; Kananavicius, R.; Barbato, R.; Korppi-Tommola, J. Excitation Energy Transfer in the LHC-II Trimer: A Model Based on the New $2.72 \AA$ Structure. Photosynth. Res. 2006, 87, 267-279.

(438) Müh, F.; Madjet, M. E.-A.; Renger, T. Structure-Based Identification of Energy Sinks in Plant Light-Harvesting Complex II. J. Phys. Chem. B 2010, 114, 1351713535.

(439) König, C.; Neugebauer, J. First-Principles Calculation of Electronic Spectra of Light-Harvesting Complex II. Phys. Chem. Chem. Phys. 2011, 13, 10475-10490.

(440) Renger, T.; Madjet, M. E.; Knorr, A.; Müh, F. How the Molecular Structure Determines the Flow of Excitation Energy in Plant Light-Harvesting Complex II. J. Plant Physiol. 2011, 168, 1497-1509.

(441) Müh, F.; Renger, T. Refined Structure-Based Simulation of Plant LightHarvesting Complex II: Linear Optical Spectra of Trimers and Aggregates. Biochim. Biophys. Acta 2012, 1817, 1446-1460.

(442) Zucchelli, G.; Santabarbara, S.; Jennings, R. C. The Qy Absorption Spectrum of the Light-Harvesting Complex II as Determined by Structure-Based Analysis of Chlorophyll Macrocycle Deformations. Biochemistry 2012, 51, 2717-2736.

(443) Dreuw, A.; Harbach, P. H. P.; Mewes, J. M.; Wormit, M. Quantum Chemical Excited State Calculations on Pigment-protein Complexes Require Thorough Geometry Re-Optimization of Experimental Crystal Structures. Theor. Chem. Acc. 2010, 125, 419-426.

(444) Calhoun, T. R.; Ginsberg, N. S.; Schlau-Cohen, G. S.; Cheng, Y.-C.; Ballottari, 
M.; Bassi, R.; Fleming, G. R. Quantum Coherence Enabled Determination of the Energy Landscape in Light Harvesting Complex II. J. Phys. Chem. B 2009, 113, 16291-16295.

(445) Novoderezhkin, V. I.; Palacios, M. A.; van Amerongen, H.; van Grondelle, R. Excitation Dynamics in the LHCII Complex of Higher Plants: Modeling Based on the 2.72 Angstrom Crystal Structure. J. Phys. Chem. B 2005, 109, 1049310504.

(446) Müh, F.; Lindorfer, D.; Schmidt am Busch, M.; Renger, T. Towards a StructureBased Exciton Hamiltonian for the CP29 Antenna of Photosystem II. Phys.

Chem. Chem. Phys. 2014, 16, 11848-11863.

(447) Reppert, M.; Zazubovich, V.; Dang, N. C.; Seibert, M.; Jankowiak, R. LowEnergy Chlorophyll States in the CP43 Antenna Protein Complex: Simulation of Various Optical Spectra. II. J. Phys. Chem. B 2008, 112, 9934-9947.

(448) Raszewski, G.; Renger, T. Light Harvesting in Photosystem II Core Complexes Is Limited by the Transfer to the Trap: Can the Core Complex Turn into a Photoprotective Mode? J. Am. Chem. Soc. 2008, 130, 4431-4446.

(449) Müh, F.; Madjet, M. E.-A.; Renger, T. Structure-Based Simulation of Linear Optical Spectra of the CP43 Core Antenna of Photosystem II. Photosynth. Res. 2012, 111, 87-101.

(450) Müh, F.; Plöckinger, M.; Ortmayer, H.; Schmidt am Busch, M.; Lindorfer, D.; Adolphs, J.; Renger, T. The Quest for Energy Traps in the CP43 Antenna of Photosystem II. J. Photochem. Photobiol. B Biol. 2015, 152, 286-300.

(451) Kröner, D.; Götze, J. P. Modeling of a Violaxanthin-Chlorophyll B Chromophore Pair in Its LHCII Environment Using CAM-B3LYP. J. Photochem. Photobiol. B. 2012, 109, 12-19.

(452) Duffy, C. D. P.; Valkunas, L.; Ruban, A. V. Quantum Mechanical Calculations of Xanthophyll-Chlorophyll Electronic Coupling in the Light-Harvesting Antenna of Photosystem II of Higher Plants. J. Phys. Chem. B 2013, 117, 76057614.

(453) Götze, J. P.; Kröner, D.; Banerjee, S.; Karasulu, B.; Thiel, W. Carotenoids as a Shortcut for Chlorophyll Soret-to-Q Band Energy Flow. ChemPhysChem 2014, $15,3392-3401$.

(454) Dreuw, A.; Fleming, G. R. R.; Head-Gordon, M. Charge-Transfer State As A Possible Signature Of A Zeaxanthin-Chlorophyll Dimer In The NonPhotochemical Quenching Process In Green Plants. J. Phys. Chem. B 2003, 107, 6500-6503.

(455) Dreuw, A.; Fleming, G. R.; Head-Gordon, M. Chlorophyll Fluorescence Quenching by Xanthophylls. Phys. Chem. Chem. Phys. 2003, 5, 3247-3256.

(456) Dreuw, A.; Fleming, G. R.; Head-Gordon, M. Role of Electron-Transfer Quenching of Chlorophyll Fluorescence by Carotenoids in Non-Photochemical Quenching of Green Plants. Biochem. Soc. Trans. 2005, 33, 858-862.

(457) Amarie, S.; Standfuss, J.; Barros, T.; Kühlbrandt, W.; Dreuw, A.; Wachtveitl, J. Carotenoid Radical Cations as a Probe for the Molecular Mechanism of Nonphotochemical Quenching in Oxygenic Photosynthesis. J. Phys. Chem. B 2007, 111, 3481-3487.

(458) Wormit, M.; Dreuw, A. Quantum Chemical Insights in Energy Dissipation and Carotenoid Radical Cation Formation in Light Harvesting Complexes. Phys. Chem. Chem. Phys. 2007, 9, 2917-2931.

(459) Dreuw, A.; Wormit, M. Simple Replacement of Violaxanthin by Zeaxanthin in LHC-II Does Not Cause Chlorophyll Fluorescence Quenching. J. Inorg. 
Biochem. 2008, 102, 458-465.

(460) Wormit, M.; Harbach, P. H. P.; Mewes, J. M.; Amarie, S.; Wachtveitl, J.; Dreuw, A. Excitation Energy Transfer and Carotenoid Radical Cation Formation in Light Harvesting Complexes - A Theoretical Perspective. Biochim. Biophys. Acta Bioenerg. 2009, 1787, 738-746.

(461) Holt, N. E.; Zigmantas, D.; Valkunas, L.; Li, X.-P.; Niyogi, K. K.; Fleming, G. R. Carotenoid Cation Formation and the Regulation of Photosynthetic Light Harvesting. Science 2005, 307, 433-436.

(462) Duffy, C. D. P.; Chmeliov, J.; Macernis, M.; Sulskus, J.; Valkunas, L.; Ruban, A. V. Modeling of Fluorescence Quenching by Lutein in the Plant Light-Harvesting Complex LHCII. J. Phys. Chem. B 2012, 117, 10974-10986.

(463) Bode, S.; Quentmeier, C. C.; Liao, P.-N.; Hafi, N.; Barros, T.; Wilk, L.; Bittner, F.; Walla, P. J. On the Regulation of Photosynthesis by Excitonic Interactions between Carotenoids and Chlorophylls. Proc. Natl. Acad. Sci. U. S. A. 2009, 106, 12311-12316.

(464) Duffy, C. D. P.; Ruban, A. V; Barford, W. Theoretical Investigation of the Role of Strongly Coupled Chlorophyll Dimers in Photoprotection of LHCII. J. Phys. Chem. B 2008, 112, 12508-12515.

(465) Blankenship, R. E. Molecular Mechanisms of Photosynthesis; Wiley-Blackwell: Oxford, 2002.

(466) Kärkäs, M. D.; Verho, O.; Johnston, E. V; Åkermark, B. Artificial Photosynthesis: Molecular Systems for Catalytic Water Oxidation. Chem. Rev. 2014, 114, 11863-12001.

(467) Zinth, W.; Wachtveitl, J. The First Picoseconds in Bacterial Photosynthesis Ultrafast Electron Transfer for the Efficient Conversion of Light Energy. ChemPhysChem 2005, 6, 871-880.

(468) Deisenhofer, J.; Epp, O.; Miki, K.; Huber, R.; Michel, H. X-Ray Structure Analysis of a Membrane Protein Complex. J. Mol. Biol. 1984, 180, 385-398.

(469) Deisenhofer, J.; Epp, O.; Miki, K.; Huber, R.; Michel, H. Structure of the Protein Subunits in the Photosynthetic Reaction Centre of Rhodopseudomonas Viridis at 3 [angst] Resolution. Nature 1985, 318, 618-624.

(470) Warshel, A. On the Origin of the Red Shift of the Absorption Spectra of Aggregated Chlorophylls. J. Am. Chem. Soc. 1979, 101, 744-746.

(471) Hanson, L. K.; Fajer, J.; Thompson, M. A.; Zerner, M. C. Electrochromic Effects of Charge Separation in Bacterial Photosynthesis: Theoretical Models. J. Am. Chem. Soc. 1987, 109, 4728-4730.

(472) Källebring, B.; Larsson, S. Singlet Spectra of Chromophores in the Reaction Center of Rhodopseudomonas Viridis as Calculated by the CNDO/S Method. Chem. Phys. Lett. 1987, 138, 76-82.

(473) Warshel, A.; Parson, W. W. Spectroscopic Properties of Photosynthetic Reaction Centers. 1. Theory. J. Am. Chem. Soc. 1987, 109, 6143-6152.

(474) Parson, W. W.; Warshel, A. Spectroscopic Properties of Photosynthetic Reaction Centers. 2. Application of the Theory to Rhodopseudomonas Viridis. J. Am. Chem. Soc. 1987, 109, 6152-6163.

(475) Eccles, J.; Honig, B.; Schulten, K. Spectroscopic Determinants in the Reaction Center of Rhodopseudomonas Viridis. Biophys. J. 1988, 53, 137-144.

(476) Thompson, M. A.; Zerner, M. C.; Fajer, J. Electronic Structure of Bacteriochlorophyll Dimers. Bacteriochlorin Models. J.Phys.Chem. 1990, 94, 3820-3828.

(477) Thompson, M. A.; Zerner, M. C. A Theoretical Examination of the Electronic 
Structure and Spectroscopy of the Photosynthetic Reaction Center from Rhodopseudomonas Viridis. J. Am. Chem. Soc. 1991, 113, 8210-8215.

(478) Thompson, M. A.; Schenter, G. K. Excited States of the Bacteriochlorophyll B Dimer of Rhodopseudomonas Viridis: A QM/MM Study of the Photosynthetic Reaction Center That Includes MM Polarization. J. Phys. Chem. 1995, 99, 63746386.

(479) Ritz, T.; Hu, X.; Damjanović, A.; Schulten, K. Excitons and Excitation Transfer in the Photosynthetic Unit of Purple Bacteria. J. Lumin. 1998, 76-77, 310-321.

(480) Damjanovic, A.; Ritz, T.; Schulten, K. Excitation Energy Trapping by the Reaction Center of Rhodobacter Sphaeroides. Int. J. Quantum Chem. 2000, 77, 139-151.

(481) Hasegawa, J.; Ohkawa, K.; Nakatsuji, H. Excited States of the Photosynthetic Reaction Center of Rhodopseudomonas Viridis: SAC-CI Study. J. Phys. Chem. B 1998, 102, 10410-10419.

(482) Nakatsuji, H.; Hasegawa, J.; Ohkawa, K. Excited States and Electron Transfer Mechanism in the Photosynthetic Reaction Center of Rhodopseudomonas Viridis: SAC-CI Study. Chem. Phys. Lett. 1998, 296, 499-504.

(483) Hasegawa, J.; Nakatsuji, H. Excited States and Electron-Transfer Mechanism in the Photosynthetic Reaction Center of Rhodobactor Sphaeroides: SAC-CI Theoretical Study. Chem. Lett. 2005, 34, 1242-1243.

(484) Renger, T.; Schlodder, E. Optical Properties, Excitation Energy and Primary Charge Transfer in Photosystem II: Theory Meets Experiment. J. Photochem. Photobiol., B 2011, 104, 126-141.

(485) Durrant, J. R.; Klug, D. R.; Kwa, S. L.; van Grondelle, R.; Porter, G.; Dekker, J. P. A Multimer Model for P680, the Primary Electron Donor of Photosystem II. Proc. Natl. Acad. Sci. U. S. A. 1995, 92, 4798-4802.

(486) Raszewski, G.; Saenger, W.; Renger, T. Theory of Optical Spectra of Photosystem II Reaction Centers: Location of the Triplet State and the Identity of the Primary Electron Donor. Biophys. J. 2005, 88, 986-998.

(487) Raszewski, G.; Diner, B. A.; Schlodder, E.; Renger, T. Spectroscopic Properties of Reaction Center Pigments in Photosystem II Core Complexes: Revision of the Multimer Model. Biophys. J. 2008, 95, 105-119.

(488) Renger, T.; Schlodder, E. Primary Photophysical Processes in Photosystem II: Bridging the Gap between Crystal Structure and Optical Spectra. ChemPhysChem 2010, 11, 1141-1153.

(489) Madjet, M. E.-A.; Müh, F.; Renger, T. Deciphering the Influence of Short-Range Electronic Couplings on Optical Properties of Molecular Dimers: Application to "Special Pairs" in Photosynthesis. J. Phys. Chem. B 2009, 113, 12603-12614.

(490) Konermann, L.; Holzwarth, A. R. Analysis of the Absorption Spectrum of Photosystem II Reaction Centers: Temperature Dependence, Pigment Assignment, and Inhomogeneous Broadening. Biochemistry 1996, 35, 829-842.

(491) Ivashin, N.; Larsson, S. Excitonic States in Photosystem II Reaction Center. J. Phys. Chem. B 2005, 109, 23051-23060.

(492) Palencar, P.; Prudnikova, T.; Vacha, F.; Kuty, M. The Effects of Light-Induced Reduction of the Photosystem II Reaction Center. J. Mol. Model. 2009, 15, 923933.

(493) Kitagawa, Y.; Matsuda, K.; Hasegawa, J. Theoretical Study of the Excited States of the Photosynthetic Reaction Center in Photosystem II: Electronic Structure, Interactions, and Their Origin. Biophys. Chem. 2011, 159, 227-236.

(494) Zhang, L.; Silva, D.-A.; Zhang, H.; Yue, A.; Yan, Y.; Huang, X. Dynamic 
Protein Conformations Preferentially Drive Energy Transfer along the Active Chain of the Photosystem II Reaction Centre. Nat. Commun. 2014, 5, 4170.

(495) Frankcombe, T. J. Explicit Calculation of the Excited Electronic States of the Photosystem II Reaction Centre. Phys. Chem. Chem. Phys. 2015, 17, 3295-3302.

(496) Stowell, M. H. Light-Induced Structural Changes in Photosynthetic Reaction Center: Implications for Mechanism of Electron-Proton Transfer. Science 1997, 276, 812-816.

(497) Jordan, P.; Fromme, P.; Witt, H. T.; Klukas, O.; Saenger, W.; Krauss, N. ThreeDimensional Structure of Cyanobacterial Photosystem I at 2.5 A Resolution. Nature 2001, 411, 909-917.

(498) Damjanović, A.; Vaswani, H. M.; Fromme, P.; Fleming, G. R. Chlorophyll Excitations in Photosystem I of Synechococcus Elongatus. J. Phys. Chem. B 2002, 106, 10251-10262.

(499) Byrdin, M.; Jordan, P.; Krauss, N.; Fromme, P.; Stehlik, D.; Schlodder, E. Light Harvesting in Photosystem I: Modeling Based on the 2.5- $\AA$ Structure of Photosystem I from Synechococcus Elongatus. Biophys. J. 2002, 83, 433-457.

(500) Sener, M. K.; Lu, D.; Ritz, T.; Park, S.; Fromme, P.; Schulten, K. Robustness and Optimality of Light Harvesting in Cyanobacterial Photosystem I. J. Phys. Chem. B 2002, 106, 7948-7960.

(501) Yang, M.; Damjanović, A.; Vaswani, H. M.; Fleming, G. R. Energy Transfer in Photosystem I of Cyanobacteria Synechococcus Elongatus: Model Study with Structure-Based Semi-Empirical Hamiltonian and Experimental Spectral Density. Biophys. J. 2003, 85, 140-158.

(502) Yin, S.; Dahlbom, M. G.; Canfield, P. J.; Hush, N. S.; Kobayashi, R.; Reimers, J. R. Assignment of the Qy, Absorption Spectrum of Photosystem-I from Thermosynechococcus Elongatus Based on CAM-B3LYP Calculations at the PW91-Optimized Protein Structure. J. Phys. Chem. B 2007, 111, 9923-9930.

(503) Adolphs, J.; Muh, F.; Madjet, M. E.-A.; Schmidt am Busch, M.; Renger, T. Structure-Based Calculations of Optical Spectra of Photosystem I Suggest an Asymmetric Light-Harvesting Process. J. Am. Chem. Soc. 2010, 132, 3331-3343.

(504) Renger, T.; Müh, F. Theory of Excitonic Couplings in Dielectric Media : Foundation of Poisson-TrEsp Method and Application to Photosystem I Trimers. Photosynth. Res. 2012, 111, 47-52.

(505) Ciamician, G. The Photochemistry of the Future. Science 1912, 36, 385-394.

(506) Jurinovich, S.; Viani, L.; Curutchet, C.; Mennucci, B. Limits and Potentials of Quantum Chemical Methods in Modelling Photosynthetic Antennae. Phys. Chem. Chem. Phys. 2015, 17, 30783-30792.

(507) Akimov, A. V.; Prezhdo, O. V. Large-Scale Computations in Chemistry: A Bird's Eye View of a Vibrant Field. Chem. Rev. 2015, 115, 5797-5890.

(508) Sisto, A.; Glowacki, D. R.; Martinez, T. J. Ab Initio Nonadiabatic Dynamics of Multichromophore Complexes: A Scalable Graphical-Processing-UnitAccelerated Exciton Framework. Acc. Chem. Res. 2014, 47, 2857-2866. 